\title{
Existence of strong solutions with critical regularity to a polytropic model for radiating flows
}

\author{
Raphaël Danchin ${ }^{1}$ • Bernard Ducomet ${ }^{2}$
}

Received: 5 February 2016 / Accepted: 1 April 2016 / Published online: 13 April 2016

(C) Fondazione Annali di Matematica Pura ed Applicata and Springer-Verlag Berlin Heidelberg 2016

\begin{abstract}
This paper is the continuation of our recent work Danchin and Ducomet (J Evol Equ 14:155-195, 2013) devoted to barotropic radiating flows. We here aim at investigating the more physically relevant situation of polytropic flows. More precisely, we consider a model arising in radiation hydrodynamics which is based on the full Navier-Stokes-Fourier system describing the macroscopic fluid motion, and a P1-approximation (see below) of the transport equation modeling the propagation of radiative intensity. In the strongly underrelativistic situation, we establish the global-in-time existence and uniqueness of solutions with critical regularity for the associated Cauchy problem with initial data close to a stable radiative equilibrium. We also justify the nonrelativistic limit in that context. For smoother (possibly) large data bounded away from the vacuum and more general physical coefficients that may depend on both the density and the temperature, the local existence of strong solutions is shown.
\end{abstract}

Keywords Radiation hydrodynamics · Under-relativistic · Polytropic Navier-Stokes system $\cdot P 1$-approximation $\cdot$ Critical spaces

Mathematics Subject Classification 35Q35 $\cdot 35 \mathrm{~B} 25 \cdot 35 \mathrm{~B} 35 \cdot 76 \mathrm{D} 05 \cdot 76 \mathrm{~N} 10$

\section{Introduction}

We consider a model of radiative fluid introduced in $[4,25,26]$. It is governed by the standard field equations of classical continuum fluid mechanics: the compressible Navier-StokesFourier system, describing the evolution of the mass density $\varrho=\varrho(t, x)$, the velocity field

Bernard Ducomet

bernard.ducomet@cea.fr

1 Université Paris-Est, LAMA (UMR 8050), UPEMLV, UPEC, CNRS, Institut Universitaire de France, 61 avenue du Général de Gaulle, 94010 Créteil Cedex 10, France

2 CEA, DAM, DIF, 91297 Arpajon, France 
$\vec{u}=\vec{u}(t, x)$, and the absolute temperature $\vartheta=\vartheta(t, x)$ as functions of the time $t$ and of the Eulerian spatial coordinate $x$. In all that follows, we assume the fluid domain to be the whole space $\mathbb{R}^{n}$.

The effect of radiation is incorporated in a scalar quantity: the radiative intensity $I=$ $I(t, x, \vec{\omega}, v)$, depending on the direction vector $\vec{\omega} \in \mathcal{S}^{n-1}$, where $\mathcal{S}^{n-1}$ denotes the unit sphere of $\mathbb{R}^{n}$, and on the frequency $v \geq 0$. The collective effect of radiation is expressed in terms of integral means (with respect to the variables $\vec{\omega}$ and $v$ ) of quantities depending on $I$. In particular, the radiation energy $S_{E}$, the radiation momentum $\vec{F}_{R}$ and the radiation tensor $\mathbb{P}_{R}$ are given by

$$
E_{R}(t, x)=\frac{1}{c} \int_{\mathcal{S}^{n-1}} \int_{0}^{\infty} I(t, x, v, \vec{\omega}) \mathrm{d} v \mathrm{~d} \vec{\omega}, \quad \vec{F}_{R}(t, x)=\int_{\mathcal{S}^{n-1}} \int_{0}^{\infty} \vec{\omega} I(t, x, v, \vec{\omega}) \mathrm{d} v \mathrm{~d} \vec{\omega},
$$

and

$$
\mathbb{P}_{R}(t, x)=\frac{1}{c} \int_{\mathcal{S}^{n-1}} \int_{0}^{\infty} \vec{\omega} \otimes \vec{\omega} I(t, x, v, \vec{\omega}) \mathrm{d} v \mathrm{~d} \vec{\omega},
$$

where $c$ is the velocity of light.

The time evolution of $I$ is governed by a transport equation with a source term depending on the temperature $\vartheta$, while the coupling to the macroscopic motion of the fluid is achieved by extra source terms in the momentum equation evaluated by means of $I$ and $\vartheta$.

The corresponding system of equations (that is derived in, e.g., $[4,25,26])$ reads:

$$
\begin{gathered}
\partial_{t} \varrho+\operatorname{div}_{x}(\varrho \vec{u})=0 \text { in }(0, T) \times \mathbb{R}^{n} ; \\
\partial_{t}(\varrho \vec{u})+\operatorname{div}_{x}(\varrho \vec{u} \otimes \vec{u})+\nabla_{x} p(\varrho, \vartheta)=\operatorname{div}_{x} \mathbb{T}-\vec{S}_{F} \text { in }(0, T) \times \mathbb{R}^{n} ; \\
\partial_{t}\left(\varrho\left(\frac{1}{2}|\vec{u}|^{2}+e(\varrho, \vartheta)\right)\right)+\operatorname{div}_{x}\left(\varrho\left(\frac{1}{2}|\vec{u}|^{2}+e(\varrho, \vartheta)\right) \vec{u}\right) \\
+\operatorname{div}_{x}(p \vec{u}+\vec{q}-\mathbb{T} \vec{u})=-S_{E} \text { in }(0, T) \times \mathbb{R}^{n}, \\
\frac{1}{c} \partial_{t} I+\vec{\omega} \cdot \nabla_{x} I=S \text { in }(0, T) \times \mathbb{R}^{n} \times(0, \infty) \times \mathcal{S}^{n-1} .
\end{gathered}
$$

The notations $p=p(\varrho, \vartheta)$ and $e=e(\varrho, \vartheta)$ designate the gaseous pressure and internal energy, respectively, and $\mathbb{T}$ stands for the viscous stress tensor determined by Newton's rheological law

$$
\mathbb{T}=\mu\left(\nabla_{x} \vec{u}+\nabla_{x}^{t} \vec{u}\right)+\lambda \operatorname{div}_{x} \vec{u} \mathbb{I}_{n},
$$

where $\mu>0$ is the shear viscosity coefficient and $\lambda=\zeta-\frac{1}{n} \mu$. Here $\zeta \geq 0$ is the bulk viscosity coefficient. We assume the heat flux $\vec{q}$ to be given by Fourier's law

$$
\vec{q}=-\kappa \nabla_{x} \vartheta,
$$

with a strictly positive heat conductivity coefficient $\kappa$. In the applications, all those coefficients may depend on both $\varrho$ and $\vartheta$. However they will be taken temperature independent in the part of the paper dedicated to the global existence issue.

In order to simplify the presentation, we assume the internal energy $e$ to be linear (and increasing) with respect to $\vartheta$, namely

$$
\partial_{\vartheta} e=C_{v} \text { for some constant } C_{v}>0 .
$$

This leads, through Maxwell's law:

$$
\varrho^{2} \partial_{\varrho} e=p-\vartheta \partial_{\vartheta} p
$$


to a pressure law depending linearly on $\vartheta$, namely

$$
p(\varrho, \vartheta)=\pi_{0}(\varrho)+\vartheta \pi_{1}(\varrho),
$$

where $\pi_{0}$ and $\pi_{1}$ are smooth functions.

Finally, the radiative sources in the right-hand sides of (2) and (3) are given by

$$
S_{E}=\int_{\mathcal{S}^{n-1}} \int_{0}^{\infty} S(\cdot, v, \vec{\omega}) \mathrm{d} v \mathrm{~d} \vec{\omega} \text { and } \vec{S}_{F}=\frac{1}{c} \int_{\mathcal{S}^{n-1}} \int_{0}^{\infty} \vec{\omega} S(\cdot, v, \vec{\omega}) \mathrm{d} \nu \mathrm{d} \vec{\omega}
$$

with

$$
S=S_{a, e}+S_{s},
$$

and where

$$
S_{a, e}=\sigma_{a}(B(v, \vartheta)-I), \quad S_{s}=\sigma_{s}\left(\frac{1}{\left|\mathcal{S}^{n-1}\right|} \int_{\mathcal{S}^{n-1}} I(\cdot, \vec{\omega}) \mathrm{d} \vec{\omega}-I\right) .
$$

Above, $\left|\mathcal{S}^{n-1}\right|$ stands for the measure of the $(n-1)$-sphere, and $B(v, \vartheta) \geq 0$ for the equilibrium thermal distribution of radiative intensity. A physically relevant example of function $B$ is

$$
B(v, \vartheta)=\frac{2 h v^{3}}{c^{2}} \frac{1}{e^{\frac{h v}{k \vartheta}}-1},
$$

where the positive real numbers $h$ and $k$ are the Planck and Boltzmann's constants. A direct computation gives

$$
\int_{0}^{\infty} \int_{\mathcal{S}^{n-1}} B(\nu, \vartheta) \mathrm{d} \vec{\omega} d \nu=\frac{2 \pi^{4}}{15} \frac{k^{4} \vartheta^{4}}{c^{2} h^{3}}\left|\mathcal{S}^{n-1}\right|,
$$

where $\left|\mathcal{S}^{n-1}\right|=2 \frac{\pi^{n / 2}}{\Gamma(n / 2)}$ is the area of the $(n-1)$-sphere. In the present work however, it will be possible to consider more general laws. Finally, the absorption coefficient $\sigma_{a}=\sigma_{a}(\nu, \varrho, \vartheta)$ and the scattering coefficient $\sigma_{s}=\sigma_{s}(\nu, \varrho, \vartheta)$ are smooth functions that are nonnegative in the applications.

System (1-4) is supplemented with the initial conditions:

$$
\varrho(0, x)=\varrho^{0}(x), \quad \vec{u}(0, x)=\vec{u}^{0}(x), \quad \vartheta(0, x)=\vartheta^{0}(x) \text { for } x \in \mathbb{R}^{n},
$$

and

$$
I(0, x, v, \vec{\omega})=I^{0}(x, v, \vec{\omega}) \text { for } x \in \mathbb{R}^{n}, \vec{\omega} \in \mathcal{S}^{n-1}, v>0
$$

The boundary conditions at infinity (e.g., convergence to some stable constant state) will be implicitly given by the functional framework we shall work in.

System (1-15) has been investigated recently in astrophysics and laser applications (in the relativistic and inviscid case) by Lowrie, Morel and Hittinger [24], Buet and Després [3], with a special attention to asymptotic regimes. The global existence result of weak solutions has been established by Ducomet et al. [15]. The reader may also refer to Dubroca and Feugeas [16], Levermore [20], Lin [22], and Lin et al. [23] for related theoretical or numerical issues.

Various approximations of the radiative transfer equation have been developed in the recent past [2] for numerical purposes. In the present paper, we are going to consider the 
so-called $P 1$ approximation [17] consisting in expanding $I$ in terms of the angular variable $\vec{\omega}$ and keeping only the first two terms in the expansion. More precisely, we choose the ansatz

$$
I=I_{0}+\vec{I}_{1} \cdot \vec{\omega}
$$

where $I_{0}$ and $\vec{I}_{1}$ do not depend on $\vec{\omega}$.

Our work aims at extending the existence theory in critical spaces developed in [12] for the radiative barotropic Navier-Stokes system, to the more physically relevant polytropic situation. We keep on considering the P1 approximation and the so-called grey case where the transport coefficients are pure positive constants independent of the frequency $v$.

The rest of the paper unfolds as follows. In the next section, we write out the system for the P1 approximation of System (1-15) in the grey case, and state our main results: first local-in-time well posedness either for smooth data and quite general assumptions on the coefficients of the system, or in the "critical regularity framework" but for coefficients depending only on the density, and linear equilibrium distribution function; second, global existence for small perturbations of a stable constant equilibrium in the strongly underrelativistic situation. Section 3 is devoted to the spectral analysis of the linearized equations about a constant reference state. We shall in particular exhibit a necessary and sufficient linear stability condition in the low-frequency regime (which is fulfilled in the strongly relativistic regime), and prove optimal global-in-time estimates for the linearized equations. The next section is devoted to the proof of similar estimates for the so-called paralinearized system (115). Those estimates are the key to our global existence result and to the rigorous justification of the nonrelativistic limit (see Sect. 5). The last section is devoted to the proof of the local existence result for large data and rather general coefficients. We postpone in Appendix some basic material related to Fourier analysis and Besov spaces.

\section{Main results}

We focus on the "grey" case where the transport coefficients are independent of the frequency $\nu$, and assume that the radiative quantities $I, B$ and $S$ have all been integrated on frequencies. Keeping the ansatz (16) for $I$, denoting by $b$ the integrated thermal distribution and taking advantage of the identity

$$
\int_{\mathcal{S}^{n-1}} \vec{\omega} \otimes \vec{\omega} \mathrm{d} \vec{\omega}=\frac{1}{n} \mathbb{I}_{n}
$$

we see that one may replace the transport equation (4) for $I$ by the following system for $\left(I_{0}, \vec{I}_{1}\right)$ :

$$
\begin{aligned}
& \frac{1}{c} \partial_{t} I_{0}+\frac{1}{n} \operatorname{div}_{x} \vec{I}_{1}=\sigma_{a}\left(b-I_{0}\right), \\
& \frac{1}{c} \partial_{t} \vec{I}_{1}+\nabla_{x} I_{0}=-\left(\sigma_{a}+\sigma_{s}\right) \vec{I}_{1} .
\end{aligned}
$$

Here we used the fact that the averaged radiative source is given by

$$
s(\varrho, \vartheta, I):=\sigma_{a}(b-I)+\sigma_{s}(<I>-I) \text { with } \quad<I>:=\frac{1}{\left|\mathcal{S}^{n-1}\right|} \int_{\mathcal{S}^{n-1}} I(t, x, \vec{\omega}) \mathrm{d} \vec{\omega} .
$$

Next, because the integrated radiative energy and momentum are given by

$$
s_{E}=\int_{\mathcal{S}^{n-1}} s \mathrm{~d} \vec{\omega} \text { and } \vec{s}_{F}=\int_{\mathcal{S}^{n-1}} \vec{\omega} s \mathrm{~d} \vec{\omega}
$$


we get, remembering (7) and (8),

$$
\begin{aligned}
& \partial_{t} \varrho+\vec{u} \cdot \nabla_{x} \varrho+\varrho \operatorname{div}_{x} \vec{u}=0, \\
& \varrho\left(\partial_{t} \vec{u}+\vec{u} \cdot \nabla_{x} \vec{u}\right)+\nabla_{x} p=2 \operatorname{div}_{x}\left(\mu D_{x} \vec{u}\right)+\nabla_{x}\left(\lambda \operatorname{div}_{x} \vec{u}\right)-\left(\frac{\sigma_{a}+\sigma_{s}}{n}\right) \vec{I}_{1}, \\
& \varrho C_{v}\left(\partial_{t} \vartheta+\vec{u} \cdot \nabla_{x} \vartheta\right)-\operatorname{div}_{x}\left(\kappa \nabla_{x} \vartheta\right) \\
& =2 \mu D_{x} \vec{u}: D_{x} \vec{u}+\lambda\left(\operatorname{div}_{x} \vec{u}\right)^{2}-\vartheta \partial_{\vartheta} p \operatorname{div}_{x} \vec{u}-\sigma_{a}\left(b(\vartheta)-I_{0}\right)+\left(\frac{\sigma_{a}+\sigma_{s}}{n}\right) \vec{I}_{1} \cdot \vec{u},
\end{aligned}
$$

where $\left(D_{x} \vec{u}\right)_{i j}:=\frac{1}{2}\left(\partial_{i} u_{j}+\partial_{j} u_{i}\right)$ and $p$ is given by (9).

Recall that a physically relevant example of thermal distribution $B$ is given by (13) which, after suitable renormalization, recasts in

$$
B(\nu, \vartheta)=\frac{15}{\left|\mathcal{S}^{n-1}\right| \pi^{4}} \frac{1}{e^{\frac{v}{\vartheta}}-1} .
$$

In this new setting, the integral on frequencies is $b(\vartheta)=\vartheta^{4}$. Our approach enables us to consider much more general functions $b$, though.

\subsection{Local results}

The local-in-time well-posedness theory does not require much assumptions on the coefficients nor on the functions in System (17-21) (apart from enough smoothness). We do not even need the radiation coefficients to be positive. In fact, we will establish in any dimension $n \geq 1$ the following basic local-in-time existence result: ${ }^{1}$

Theorem 2.1 Assume that $b$ depends smoothly on the temperature, and that $\lambda, \mu, \kappa, \sigma_{a}$ and $\sigma_{s}$ are smooth functions of the density and of the temperature. If in addition

$$
\mu>0, \quad \lambda+2 \mu>0 \text { and } \kappa>0,
$$

then for any data $\varrho^{0}, \vec{u}^{0}, \vartheta^{0}, I_{0}^{0}$ and $\vec{I}_{1}^{0}$ satisfying

(1) $\varrho^{0}$ and $\vartheta^{0}$ are bounded, and bounded away from 0 ,

(2) $\vec{I}_{1}^{0}, \nabla \varrho^{0}$ and $\vec{u}^{0}$ are in $B_{2,1}^{\frac{n}{2}}$,

(3) $\Theta^{0}:=\vartheta^{0}-\bar{\vartheta}$ is in $B_{2,1}^{\frac{n}{2}}$ for some positive constant $\bar{\vartheta}$,

(4) $I_{0}^{0}=b(\bar{\vartheta})+j_{0}^{0}$ with $j_{0}^{0}$ in $B_{2,1}^{\frac{n}{2}}$,

there exists $T>0$ so that System (17-21) with pressure law (9) and data $\left(\varrho^{0}, \vec{u}^{0}, \vartheta^{0}, I_{0}^{0}, \vec{I}_{1}^{0}\right)$ admits a unique local solution $\left(\varrho, \vec{u}, \vartheta, I_{0}, \vec{I}_{1}\right)$ on $[0, T] \times \mathbb{R}^{n}$ with

(1) $\vartheta^{ \pm 1}$ and $\varrho^{ \pm 1}$ in $\mathcal{C}_{b}\left([0, T] \times \mathbb{R}^{n}\right)$ and $\nabla \varrho \in \mathcal{C}\left([0, T] ; B_{2,1}^{\frac{n}{2}}\right)$,

(2) $\vec{u}$ and $\Theta:=\vartheta-\bar{\vartheta}$ in $\mathcal{C}\left([0, T] ; B_{2,1}^{\frac{n}{2}}\right) \cap L^{1}\left(0, T ; B_{2,1}^{\frac{n}{2}+2}\right)$,

(3) $j_{0}=I_{0}-b(\bar{\vartheta})$ and $\vec{I}_{1}$ in $\mathcal{C}\left([0, T] ; B_{2,1}^{\frac{n}{2}}\right)$.

Remark 2.1 Resorting to more elaborate arguments (like Proposition 6 of [9]), it should be possible to consider density with the same regularity as the other data, instead of one

$1^{1}$ See the Appendix for the definition of nonhomogeneous Besov spaces $B_{2,1}^{S}$. 
more derivative. In fact, having more regular density is helpful in parabolic estimates as the second-order terms have coefficients depending on both $\varrho$ and $\vartheta$, and no gain of smoothness is obtained for $\varrho$, through the evolution. Of course, it is possible to propagate higher Besov (or Sobolev) regularity, provided it is related to the $L^{2}$ space.

Although a bit technical, the proof of Theorem 2.1 relies on completely standard arguments: basic estimates for the transport equation (as regards the density), parabolic equations or systems (temperature and velocity) and hyperbolic symmetric systems with constant coefficients (radiative equations). High regularity is needed just to handle the dependency of the coefficients of the system on both $\varrho$ and $\vartheta$. Roughly speaking, composition lemmas are nicer in spaces embedded in the set of bounded functions (see the Appendix), which in the Besov spaces scale $B_{2,1}^{s}$, is equivalent to $s \geq \frac{n}{2}$.

Let us now go to the case where the coefficients depend only on $\varrho$, and where $b$ depends linearly on $\vartheta$. Then the critical regularity framework becomes relevant for solving System $(17-21)$. As in the nonradiative case studied in [6,7], critical norms for $(\varrho, \vec{u}, \vartheta)$ are invariant for all $\ell>0$ by the following scaling transformation:

$$
(\varrho(t, x), \vec{u}(t, x), \vartheta(t, x)) \rightsquigarrow\left(\varrho\left(\ell^{2} t, \ell x\right), \ell \vec{u}\left(\ell^{2} t, \ell x\right), \ell^{2} \vartheta\left(\ell^{2} t, \ell x\right)\right)
$$

which leaves the density, velocity and temperature equations invariant, up to a suitable change of the pressure law.

Although the radiative unknowns do not have any natural scaling invariance, the coupling between hydrodynamic and radiative unknowns forces us to work at the same level of regularity as for the velocity.

To be more specific, let us fix some reference positive constant density $\bar{\varrho}$ and temperature $\bar{\vartheta}$, and set $\bar{b}:=b(\bar{\vartheta})$. Keeping in mind that the pressure is given by (9), the system for $a:=\varrho-\bar{\varrho}, \vec{u}, \Theta:=\vartheta-\bar{\vartheta}, j_{0}:=I_{0}-\bar{b}$ and $\vec{j}_{1}:=\vec{I}_{1}$ reads:

$$
\left\{\begin{array}{l}
\partial_{t} a+\vec{u} \cdot \nabla_{x} a+(\bar{\varrho}+a) \operatorname{div}_{x} \vec{u}=0, \\
(\bar{\varrho}+a)\left(\partial_{t} \vec{u}+\vec{u} \cdot \nabla_{x} \vec{u}\right)+\nabla_{x}(p(\bar{\varrho}+a, \bar{\vartheta}+\Theta)) \\
=2 \operatorname{div}_{x}\left(\mu D_{x} \vec{u}\right)+\nabla_{x}\left(\lambda \operatorname{div}_{x} \vec{u}\right)-\left(\frac{\sigma_{a}+\sigma_{s}}{n}\right) \vec{j}_{1}, \\
C_{v}(\bar{\varrho}+a)\left(\partial_{t} \vartheta+\vec{u} \cdot \nabla_{x} \vartheta\right)-\operatorname{div}_{x}\left(\kappa \nabla_{x} \vartheta\right)=2 \mu D_{x} \vec{u}: D_{x} \vec{u} \\
\quad+\lambda\left(\operatorname{div}_{x} \vec{u}\right)^{2}-\vartheta \pi_{1}(\bar{\varrho}+a) \operatorname{div}_{x} \vec{u}-\sigma_{a}\left(b(\vartheta)-\bar{b}-j_{0}\right)+\left(\frac{\sigma_{a}+\sigma_{s}}{n}\right) \overrightarrow{j_{1}} \cdot \vec{u}, \\
\frac{1}{c} \partial_{t} j_{0}+\frac{1}{n} \operatorname{div}_{x} \vec{j}_{1}=\sigma_{a}\left(b-\bar{b}-j_{0}\right), \\
\frac{1}{c} \partial_{t} \vec{j}_{1}+\nabla_{x} j_{0}=-\left(\sigma_{a}+\sigma_{s}\right) \vec{j}_{1} .
\end{array}\right.
$$

To state our results, let us associate to any tempered distribution $z$ its low- and high-frequency parts denoted by $z^{\ell}$ and $z^{h}$, respectively [see the definition in (136)]. Then we have:

Theorem 2.2 Let $n \geq 3$. Assume that the data $a^{0}, \vec{u}^{0}, \vartheta^{0}, j_{0}^{0}$ and $\vec{j}_{0}^{1}$ satisfy

$$
\left(a^{0}\right)^{\ell}, \vec{u}^{0},\left(\vartheta^{0}\right)^{\ell}, j_{0}^{0}, \vec{j}_{1}^{0} \in \dot{B}_{2,1}^{\frac{n}{2}-1}, \quad\left(a^{0}\right)^{h} \in \dot{B}_{2,1}^{\frac{n}{2}} \text { and }\left(\vartheta^{0}\right)^{h} \in \dot{B}_{2,1}^{\frac{n}{2}-2} .
$$

If in addition $\bar{\varrho}+a^{0}$ is bounded away from 0 then there exists $T>0$ such that System (24) with data $\left(a^{0}, \vec{u}^{0}, \vartheta^{0}, j_{0}^{0}, \vec{j}_{1}^{0}\right)$ and pressure law (9) admits a unique local solution $\left(a, \vec{u}, \vartheta, j_{0}, \vec{j}_{1}\right)$ on $[0, T] \times \mathbb{R}^{n}$ with

(1) $a^{\ell} \in \mathcal{C}\left([0, T] ; \dot{B}_{2,1}^{\frac{n}{2}-1}\right), a^{h} \in \mathcal{C}\left([0, T] ; \dot{B}_{2,1}^{\frac{n}{2}}\right)$, and $1+$ a bounded away from 0 , 
(2) $\vec{u} \in \mathcal{C}\left([0, T] ; \dot{B}_{2,1}^{\frac{n}{2}-1}\right) \cap L^{1}\left(0, T ; \dot{B}_{2,1}^{\frac{n}{2}+1}\right)$,

(3) $\vartheta^{\ell} \in \mathcal{C}\left([0, T] ; \dot{B}_{2,1}^{\frac{n}{2}-1}\right) \cap L^{1}\left(0, T ; \dot{B}_{2,1}^{\frac{n}{2}+1}\right)$ and $\vartheta^{h} \in \mathcal{C}\left([0, T] ; \dot{B}_{2,1}^{\frac{n}{2}-2}\right) \cap L^{1}\left(0, T ; \dot{B}_{2,1}^{\frac{n}{2}}\right)$,

(4) $j_{0}$ and $\vec{j}_{1}$ in $\mathcal{C}\left([0, T] ; \dot{B}_{2,1}^{\frac{n}{2}-1}\right)$.

Let us emphasize that in contrast with the nonradiative case studied in [6], whether one may adapt the above statement to the critical $L^{p}$ framework (that is to critical Besov spaces $\left.\dot{B}_{p, 1}^{s}\left(\mathbb{R}^{n}\right)\right)$ is unclear. The reason why is that the unknowns $\left(j_{0}, \vec{j}_{1}\right)$ satisfy a symmetric hyperbolic system (the coupling with the other two equations being lower order), and solving hyperbolic systems in spaces which are not related to $L^{2}$ is not possible in general.

\subsection{Global results}

Let us now present our global well-posedness result in the critical regularity framework, in the case where the coefficients of the system depend only on the density. To find out sufficient conditions for the global well posedness, it is convenient to work with the non-dimensional form of System (17-21). To this end, following [2], we fix

$$
L_{r e f}, T_{r e f}, U_{r e f}, \varrho_{r e f}, \vartheta_{r e f}, p_{r e f}, e_{r e f}, \mu_{r e f}=\lambda_{r e f}, \kappa_{r e f},
$$

some reference hydrodynamical quantities (length, time, velocity, density, temperature, pressure, energy, viscosity, conductivity), and

$$
I_{\text {ref }}, \sigma_{a, r e f}, \sigma_{s, r e f},
$$

the reference radiative quantities (radiative intensity, absorption and scattering coefficients). Then we put

$$
\begin{aligned}
& \widehat{x}=\frac{x}{L_{r e f}}, \quad \widehat{t}=\frac{t}{T_{r e f}}, \quad \widehat{\varrho}=\frac{\varrho}{\varrho_{r e f}}, \quad \widehat{\vartheta}=\frac{\vartheta}{\vartheta_{r e f}}, \quad \widehat{p}=\frac{p}{\varrho_{r e f} U_{r e f}^{2}}, \\
& \widehat{I}=\frac{k I}{a_{r} c h \vartheta_{r e f}^{3}} \quad \text { with } a_{r}=\frac{2 \pi^{4}\left|\mathcal{S}^{n-1}\right| k^{4}}{15 c^{3} h^{3}} .
\end{aligned}
$$

Set $C_{p}:=C_{v}+\frac{\vartheta\left(\partial_{\vartheta} p\right)^{2}}{\varrho^{2} \partial_{\varrho} p} \cdot$ Let

$$
S r=\frac{L_{r e f}}{T_{r e f} U_{r e f}}, \quad M a=\frac{U_{r e f}}{\sqrt{p_{\text {ref }} / \varrho_{r e f}}}, \quad R e=\frac{U_{r e f} \varrho_{r e f} L_{r e f}}{\mu_{r e f}}, \quad \operatorname{Pr}=\frac{C_{p_{r e f}} \mu_{r e f}}{\kappa_{r e f}}
$$

be the Strouhal, Mach, Reynolds, Prandtl (dimensionless) numbers corresponding to hydrodynamics, and

$$
\mathcal{C}=\frac{c}{U_{\text {ref }}}, \quad \mathcal{L}=L_{\text {ref }} \sigma_{a, r e f}, \quad \mathcal{L}_{s}=\frac{\sigma_{s, r e f}}{\sigma_{a, r e f}}, \quad \mathcal{P}=\frac{a_{r} \vartheta_{r e f}^{4}}{\varrho_{r e f} U_{r e f}^{2}},
$$

be dimensionless numbers corresponding to radiation.

In the following, we suppose for simplicity that $S r=\mathcal{P}=1$ (that is the radiative energy is comparable to the kinetic energy). We also have to keep in mind that only the situation where $\mathcal{C} \gg 1$ is relevant in our model, for the matter is treated classically.

Considering the reference equilibrium $\varrho=\varrho_{\text {ref }}, \vec{u}=\overrightarrow{0}, \vartheta=\vartheta_{\text {ref }}, I_{0}=b\left(\vartheta_{\text {ref }}\right), \vec{I}_{1}=\overrightarrow{0}$, that corresponds, after rescaling, to

$$
\widehat{\varrho}=1, \widehat{\vec{u}}=\overrightarrow{0}, \widehat{\vartheta}=1, \widehat{I}_{0}=b(\bar{\vartheta}), \widehat{\vec{I}_{1}}=\overrightarrow{0},
$$


we set (omitting the carets for notational simplicity)

$$
a:=\varrho-1, \quad \Theta:=\vartheta-1, \quad j_{0}:=I_{0}-b(1), \quad \vec{j}_{1}:=\vec{I}_{1},
$$

and eventually get the following system:

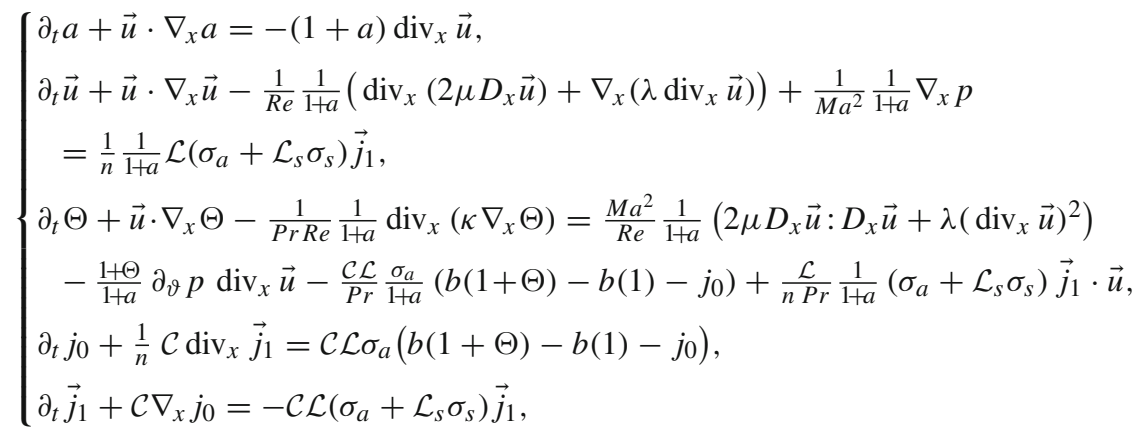

which rewrites, omitting the dependency with respect to $x$ in the differential operators from now on, and using (9),

$$
\left\{\begin{array}{l}
\partial_{t} a+\operatorname{div} \vec{u}=F-\vec{u} \cdot \nabla a, \\
\partial_{t} \vec{u}-\frac{1}{\operatorname{Re}} \underline{\mathcal{A}} \vec{u}+\frac{1}{M a^{2}} \underline{\alpha}_{1} \nabla a+\frac{1}{M a^{2}} \underline{\alpha}_{2} \nabla \Theta-\frac{1}{n} \mathcal{L}\left(\underline{\sigma}_{a}+\mathcal{L}_{s} \underline{\sigma}_{s}\right) \vec{j} 1=\vec{G}-\vec{u} \cdot \nabla \vec{u}, \\
\partial_{t} \Theta-\frac{1}{\operatorname{Pr} R e} \underline{\kappa} \Delta \Theta+\underline{\alpha}_{2} \operatorname{div} \vec{u}-\frac{\mathcal{C L}}{\operatorname{Pr}} \underline{\sigma}_{a}\left(j_{0}-\underline{\alpha}^{\prime} \Theta\right)=H-\vec{u} \cdot \nabla \Theta, \\
\partial_{t} j_{0}+\frac{1}{n} \mathcal{C} \operatorname{div} \overrightarrow{j_{1}}+\mathcal{C} \mathcal{L} \underline{\sigma}_{a}\left(j_{0}-\underline{\alpha}^{\prime} \Theta\right)=J_{0}, \\
\partial_{t} \vec{j}_{1}+\mathcal{C} \nabla j_{0}+\mathcal{C} \mathcal{L}\left(\underline{\sigma}_{a}+\mathcal{L}_{s} \underline{\sigma}_{s}\right) \vec{j}_{1}=\vec{J}_{1},
\end{array}\right.
$$

with the notations $\underline{\mathcal{A}}:=\mu \underline{\Delta}+(\underline{\lambda}+\underline{\mu}) \nabla \operatorname{div}$,

$$
\begin{aligned}
& \underline{\sigma}_{a}:=\sigma_{a}(1), \quad \underline{\sigma}_{*} * s:=\sigma_{s}(1), \quad \underline{\lambda}:=\lambda(1), \quad \mu:=\mu(1), \quad \underline{\kappa}=\kappa(1), \quad \underline{b}:=b(1), \\
& \underline{\alpha}_{1}:=\partial_{\varrho} p(1,1)=\pi_{0}^{\prime}(1)+\pi_{1}^{\prime}(1), \quad \underline{\alpha}_{2}:=\partial_{\vartheta} p(1,1)=\pi_{1}(1), \quad \underline{\alpha}^{\prime}=\partial_{\vartheta} b(1),
\end{aligned}
$$

and the right-hand sides

$$
\begin{aligned}
F:=-a \operatorname{div} \vec{u}, \\
\vec{G}:=\frac{1}{\operatorname{Re}}\left[\frac{1}{1+a}(\operatorname{div}(2 \mu D \vec{u})+\nabla(\lambda \operatorname{div} \vec{u}))-\underline{\mathcal{A}} \vec{u}\right] \\
+\frac{1}{M a^{2}}\left[\partial_{\varrho} p(1,1)-\frac{1}{1+a} \partial_{\varrho} p(1+a, 1+\Theta)\right] \nabla a \\
+\frac{1}{M a^{2}}\left[\partial_{\vartheta} p(1,1)-\frac{1}{1+a} \partial_{\vartheta} p(1+a, 1+\Theta)\right] \nabla \Theta \\
-\frac{1}{n} \mathcal{L}\left[\underline{\sigma}_{a}-\frac{\sigma_{a}}{1+a}+\mathcal{L}_{s}\left(\underline{\sigma}_{s}-\frac{\sigma_{s}}{1+a}\right)\right] \vec{j}_{1}, \\
H:=\frac{1}{\operatorname{Pr} \operatorname{Re}} \frac{1}{1+a} \operatorname{div}((\kappa-\underline{\kappa}) \nabla \Theta)-\left[(1+\Theta) \frac{\pi_{1}(1+a)}{1+a}-\pi_{1}(1)\right] \operatorname{div} \vec{u} \\
+\frac{\mathcal{L}}{n \operatorname{Pr}} \frac{1}{1+a}\left(\sigma_{a}+\mathcal{L}_{s} \sigma_{s}\right) \vec{j}_{1} \cdot \vec{u} \\
+\frac{\mathcal{C} \mathcal{L}}{\operatorname{Pr}}\left(\frac{\sigma_{a}}{1+a}-\underline{\sigma}_{a}\right)\left(j_{0}-\underline{\alpha}^{\prime} \Theta\right)+\frac{M a^{2}}{\operatorname{Re}} \frac{1}{1+a}\left(2 \mu D \vec{u}: D \vec{u}+\lambda(\operatorname{div} \vec{u})^{2}\right)
\end{aligned}
$$




$$
\begin{aligned}
J_{0} & \left.:=\mathcal{C} \mathcal{L}\left[\sigma_{a}(b(1+\Theta)-\underline{b})-\underline{\alpha}^{\prime} \Theta\right)+\left(\sigma_{a}-\sigma_{a}\right)\left(j_{0}-\underline{\alpha}^{\prime} \Theta\right)\right], \\
\vec{J}_{1} & :=-\mathcal{C} \mathcal{L}\left[\sigma_{a}-\underline{\sigma}_{a}+\mathcal{L}_{s}\left(\sigma_{s}-\underline{\sigma}_{s}\right)\right] \vec{j}_{1} .
\end{aligned}
$$

Constructing global strong solutions for (25) in the case of small data with critical regularity is the second (and main) purpose of the present paper. Before giving the statement, let us introduce the solution space: we denote by $E^{s}$ the set of functions $\left(a, \vec{u}, \Theta, j_{0}, \vec{j}_{1}\right)$ so that ${ }^{2}$

$$
\begin{aligned}
& a^{\ell} \in \mathcal{C}_{b}\left(\mathbb{R}^{+} ; \dot{B}_{2,1}^{s}\right) \cap L^{1}\left(\mathbb{R}^{+} ; \dot{B}_{2,1}^{s+2}\right) \quad \text { and } a^{h} \in \mathcal{C}_{b}\left(\mathbb{R}^{+} ; \dot{B}_{2,1}^{s+1}\right) \cap L^{1}\left(\mathbb{R}^{+} ; \dot{B}_{2,1}^{s+1}\right) \\
& \quad j_{0}^{\ell}, \vec{j}_{1}^{\ell}, \vec{u} \in \mathcal{C}_{b}\left(\mathbb{R}^{+} ; \dot{B}_{2,1}^{s}\right) \cap L^{1}\left(\mathbb{R}^{+} ; \dot{B}_{2,1}^{s+2}\right) \\
& j_{0}^{h}, \vec{j}_{1}^{h} \in \mathcal{C}_{b}\left(\mathbb{R}^{+} ; \dot{B}_{2,1}^{s}\right) \cap L^{1}\left(\mathbb{R}^{+} ; \dot{B}_{2,1}^{s}\right), \\
& \Theta^{\ell} \in \mathcal{C}_{b}\left(\mathbb{R}^{+} ; \dot{B}_{2,1}^{s}\right) \cap L^{1}\left(\mathbb{R}^{+} ; \dot{B}_{2,1}^{s+2}\right) \text { and } \Theta^{h} \in \mathcal{C}_{b}\left(\mathbb{R}^{+} ; \dot{B}_{2,1}^{s-1}\right) \cap L^{1}\left(\mathbb{R}^{+} ; \dot{B}_{2,1}^{s+1}\right) .
\end{aligned}
$$

The following result states that for strongly under-relativistic fluids and small data, global existence of strong critical solutions to (25) holds true.

Theorem 2.3 Let $n \geq 3$. Assume that $\lambda, \mu, \kappa$ and $\sigma_{s}$ depend smoothly on $\varrho$ [with $\lambda, \mu$, $\kappa$ satisfying (22)], and that $\sigma_{a}$ is a positive constant. Suppose that the thermal distribution function $b$ depends linearly on $\vartheta$.

There exist a large constant $\mathcal{C}_{0}>0$ and a small constant $c>0$ depending only on the dimension $n$ and on the rescaled parameters of the system, such that if

$$
\mathcal{C} \geq \mathcal{C}_{0},
$$

and the initial data $a^{0}, \vec{u}^{0}, \Theta^{0}, j_{0}^{0}$ and $\vec{j}_{1}^{0}$ satisfy the smallness condition

$$
X_{0}:=\left\|\left(\vec{u}^{0}, j_{0}^{0}, \vec{j}_{1}^{0}\right)\right\|_{\dot{B}_{2,1}^{\frac{n}{2}-1}}+\left\|\left(\Theta^{0}, a^{0}\right)\right\|_{\dot{B}_{2,1}^{\frac{n}{2}-1}}^{\ell}+\left\|a^{0}\right\|_{\dot{B}_{2,1}^{\frac{n}{2}}}^{h}+\left\|\Theta^{0}\right\|_{\dot{B}_{2,1}^{\frac{n}{2}-2}}^{h} \leq c,
$$

then System (25) has a unique global solution $\left(a, \vec{u}, \Theta, j_{0}, \vec{j}_{1}\right)$ in $E^{\frac{n}{2}-1}$. Besides,

$$
\left\|\left(a, \vec{u}, \Theta, j_{0}, \vec{j}_{1}\right)\right\|_{E^{\frac{n}{2}-1}} \leq K X_{0},
$$

with $K$ depending only on $n$ and on the coefficients of the system, and we have the following decay estimates:

$$
\mathcal{C} \int_{\mathbb{R}^{+}}\left(\left\|\zeta_{0}\right\|_{\dot{B}_{2,1}^{\frac{n}{2}-1}}^{\ell}+\left\|\vec{j}_{1}\right\|_{\dot{B}_{2,1}^{\frac{n}{2}-1}}+\left\|j_{0}\right\|_{\dot{B}_{2,1}^{\frac{n}{2}-1}}^{h}+\|\Theta\|_{\dot{B}_{2,1}^{2}-2}^{h}\right) \mathrm{d} t \leq K X_{0}
$$

with $\zeta_{0}:=j_{0}-\underline{\alpha}^{\prime} \Theta-\frac{\underline{\alpha}_{2} \underline{\alpha}^{\prime}}{\mathcal{C} \mathcal{L} \underline{\alpha}_{1} \underline{\sigma}_{a}\left(1+\frac{1}{\operatorname{Pr}} \underline{\alpha}^{\prime}\right)} \operatorname{div} \vec{u}$.

The proof is based on a fine analysis of the linearized equations about the reference state and on paralinearization arguments similar to those of, e.g., [1], Chap. 10 or [12], to avoid the loss of one derivative that may cause the convection terms. Let us make another comments:

(1) Exhibiting the low-frequency decay properties in (30) is absolutely essential for the proof of the global existence as it allows to get a quadratic control on the radiative terms in $H$ (defined above).

${ }^{2}$ Notations $z^{\ell}, z^{h},\|\cdot\|_{\dot{B}_{2,1}^{s}}^{\ell}$ and so on are defined in Appendix. 
(2) The stability condition (27) comes from our analysis of the linearized equations. In fact, in the low-frequency regime, we proved that the necessary and sufficient stability condition reads as follows: ${ }^{3}$

$$
\begin{gathered}
\digamma:=\frac{\mathcal{C} \mathcal{L} \sigma_{a} M a^{2}}{R e}\left(\widetilde{\alpha} \nu+\frac{\kappa}{P r}\right)+\frac{\alpha^{\prime} \alpha_{2}}{\widetilde{\alpha}}\left(\frac{\alpha_{2}}{P r}-\frac{M a^{2}}{n}\right)>0 \text { and } \\
\left(1+\frac{\alpha^{\prime} M a^{2}}{n \alpha_{2}}\right) \digamma+\frac{v \widetilde{\alpha}^{2} \sigma_{a} \alpha_{1}^{2} \mathcal{C} \mathcal{L} M a^{2}}{\alpha_{2}^{2} \operatorname{Re}}+\frac{\alpha^{\prime} \alpha_{1}}{P r}>\frac{\alpha_{1} \alpha^{\prime} M a^{2}}{n \alpha_{2}}\left(1+\frac{\widetilde{\alpha}}{1+\mathcal{L}_{s} \sigma_{s} / \sigma_{a}}\right) .
\end{gathered}
$$

We also established that for high frequencies, linear stability is true whenever (22) is satisfied and all the other coefficients entering in (26) are positive.

Unfortunately, in contrast with the barotropic case that we treated in [12], the computations for middle frequencies are so wild that we have not been able to check whether (31) does ensure linear stability, unless $\mathcal{C}$ is very large. We strongly believe however that it is the correct necessary and sufficient stability conditions for all frequencies.

(3) For any set of parameters for which the linear system given by the l.h.s. of (25) is strongly stable (i.e., the eigenvalues of the matrix of the system in Fourier variables have positive real parts), one may reproduce the decay estimates of Sect. 3. Indeed linear stability implies (51) and (22) (as we found out the necessary and sufficient stability condition in low and high frequency) and the decay estimates we proved in Sect. 3 are thus valid. Now as the set of medium frequency is compact, strong stability implies uniform exponential stability in that range (see, e.g., [12]). Then going through the computations of Sect. 5 , we get another global existence statement for small enough data (with a smallness condition depending on all the parameters of the system), and we can even afford to have some density dependent $\sigma_{a}$, as we do not care if the smallness condition depends on $\mathcal{C}$.

(4) We strongly believe that if we take smoother data (like in, e.g., Theorem 2.2), then one can get a global-in-time statement under the same stability Condition (27), even if the coefficients all depend on both the density and on the temperature, and for more general distribution function $b$ (like $\theta^{4}$ for example). The reason why we refrained from addressing that physically relevant question here is to keep the paper a reasonable size.

As a corollary of the estimates (29) and (30) pointed out in the above theorem, we get the following result for the nonrelativistic limit of (25).

Corollary 2.1 Let $\left(a_{\varepsilon}^{0}, \vec{u}_{\varepsilon}^{0}, \Theta_{\varepsilon}^{0}, j_{0, \varepsilon}^{0}, \vec{j}_{1, \varepsilon}^{0}\right)$ be a family of data fulfilling (28). Consider the corresponding family of solutions $\left(a_{\varepsilon}, \vec{u}_{\varepsilon}, \Theta_{\varepsilon}, j_{0, \varepsilon}, \vec{j}_{1, \varepsilon}\right)$ to $(25)$ with $\mathcal{C}=\varepsilon^{-1}$, provided by Theorem 2.3.

If we assume in addition that $\left(a_{\varepsilon}^{0}, \vec{u}_{\varepsilon}^{0}\right) \rightarrow\left(a^{0}, \vec{u}^{0}\right)$ in the sense of distributions when $\varepsilon \rightarrow 0$, then we have

$$
\left(a_{\varepsilon}, \vec{u}_{\varepsilon}\right) \rightarrow(a, \vec{u}) \text { and }\left(\Theta_{\varepsilon}, j_{0, \varepsilon}, \vec{j}_{1, \varepsilon}\right) \rightarrow(0,0, \overrightarrow{0}),
$$

where $(a, \vec{u}) \in\left(\mathcal{C}\left(\mathbb{R}_{+} ; \dot{B}_{2,1}^{\frac{n}{2}-1} \cap \dot{B}_{2,1}^{\frac{n}{2}}\right) \cap L^{1}\left(\mathbb{R}_{+} ; \dot{B}_{2,1}^{\frac{n}{2}}+\dot{B}_{2,1}^{\frac{n}{2}+1}\right)\right) \times\left(\mathcal{C}\left(\mathbb{R}_{+} ; \dot{B}_{2,1}^{\frac{n}{2}-1}\right) \cap\right.$ $\left.L^{1}\left(\mathbb{R}_{+} ; \dot{B}_{2,1}^{\frac{n}{2}+1}\right)\right)^{n}$ is the unique solution of the following (isothermal) compressible NavierStokes equations:

$$
\left\{\begin{array}{l}
\partial_{t} a+\vec{u} \cdot \nabla a=-(1+a) \operatorname{div}_{x} \vec{u}, \\
\partial_{t} \vec{u}+\vec{u} \cdot \nabla \vec{u}-\frac{1}{R e} \frac{1}{1+a}(\operatorname{div}(2 \mu D \vec{u})+\nabla(\lambda \operatorname{div} \vec{u}))+\frac{1}{M a^{2}} \frac{1}{1+a} \nabla\left(\pi_{0}+\pi_{1}\right)=\overrightarrow{0},
\end{array}\right.
$$

supplemented with initial data $\left(a_{0}, \vec{u}_{0}\right)$.

\footnotetext{
${ }^{3}$ Where we set $\widetilde{\alpha}=1+\alpha^{\prime} / P r$, use the notations (26) and omit the underlines for better readability.
} 


\section{Linear analysis of the $P 1$ approximation system}

This section is devoted to the linear analysis of System (25) about the null equilibrium. After noticing that the divergence free parts of $\vec{u}$ and $\vec{j}_{1}$ are uncoupled from the rest of the system, we concentrate on the linear stability of the other unknowns. The main difficulty is that the linearized equations, although with constant coefficients, involve a great number of parameters. To reduce that number to "only" nine, we perform a suitable rescaling. Then, the most intricate part of the analysis concerns the low frequencies (the high-frequency regime turns out to be easier since the radiative part of the system tends to uncouple from the hydrodynamics one). In fact, the linearized system does not enter in any standard class of partial differential equations : it does not have much structure and has terms of three different orders. We shall nevertheless succeed in implementing the method we introduced recently in $[13,14]$ in the simpler context of barotropic radiative flows so as to reduce our study to that of a nicer system up to error terms that are small in the low-frequency regime.

\subsection{The linearized $P 1$ system}

It corresponds to the 1.h.s. of (25), looking at the r.h.s. as given source terms. We shall concentrate on the case where the source terms are zero, keeping in mind that the general case may be deduced afterward from Duhamel's formula.

Let $\mathcal{P}$ and $\mathcal{Q}$ denote the Helmholtz projectors on solenoidal and potential vector fields, respectively. We notice that $\mathcal{P} \vec{u}$ and $\mathcal{P} \vec{j}_{1}$ satisfy a linear heat equation, and a damped equation, namely denoting $\underline{\beta}=\frac{1}{n}\left(\underline{\sigma}_{a}+\mathcal{L}_{s} \underline{\sigma}_{s}\right)$,

$$
\partial_{t} \mathcal{P} \vec{u}-\frac{1}{R e} \underline{\mu} \Delta \mathcal{P} \vec{u}=\underline{\beta} \mathcal{L} \mathcal{P} \vec{j}_{1} \quad \text { and } \quad \partial_{t} \mathcal{P} \vec{j}_{1}+n \underline{\beta \mathcal{C}} \mathcal{L} \mathcal{P} \vec{j}_{1}=\overrightarrow{0} .
$$

The system satisfied by $\left(a, \mathcal{Q} \vec{u}, \Theta, j_{0}, \mathcal{Q} \vec{j}_{1}\right)$ is much more involved. To work with scalar unknowns, one sets

$$
d:=\Lambda^{-1} \operatorname{div} \vec{u} \text { and } j_{1}:=\Lambda^{-1} \operatorname{div} \vec{j}_{1} \text { with } \Lambda^{ \pm 1}:=(-\Delta)^{ \pm 1 / 2} .
$$

From the point of view of a priori estimates, working with $\left(\mathcal{Q} \vec{u}, \mathcal{Q} \vec{j}_{1}\right)$ or $\left(d, j_{1}\right)$ is equivalent, since $\mathcal{Q} \vec{u}=-\Lambda^{-1} \nabla d$ and $\mathcal{Q} \vec{j}_{1}=-\Lambda^{-1} \nabla j_{1}$, and 0-th order Fourier multipliers are selfmaps on homogeneous Besov spaces.

Now the $5 \times 5$ system for $\left(a, d, \Theta, j_{0}, j_{1}\right)$ reads, putting $\underline{v}=\underline{\lambda}+2 \underline{\mu}$ :

$$
\left\{\begin{array}{l}
\partial_{t} a+\Lambda d=0, \\
\partial_{t} d-\frac{1}{\operatorname{Re}} \underline{\nu} \Delta d-\frac{1}{M a^{2}} \underline{\alpha}_{1} \Lambda a-\frac{1}{M a^{2}} \underline{\alpha}_{2} \Lambda \Theta-\mathcal{L} \underline{\beta} j_{1}=0, \\
\partial_{t} \Theta-\underline{\kappa} \frac{1}{\operatorname{Pr} R e} \Delta \Theta+\underline{\alpha}_{2} \Lambda d+\frac{\mathcal{C} \mathcal{L}}{\operatorname{Pr}} \underline{\sigma}_{a}\left(\underline{\alpha}^{\prime} \Theta-j_{0}\right)=0, \\
\partial_{t} j_{0}+\frac{1}{n} \mathcal{C} \Lambda j_{1}-\mathcal{C} \mathcal{L} \underline{\sigma}_{a}\left(\underline{\alpha}^{\prime} \Theta-j_{0}\right)=0, \\
\partial_{t} j_{1}-\mathcal{C} \Lambda j_{0}+n \mathcal{C} \mathcal{L} \underline{\beta} j_{1}=0 .
\end{array}\right.
$$

Taking the Fourier transform with respect to the space variable, the above system recasts in

$$
\mathrm{d} t\left(\begin{array}{c}
\widehat{a} \\
\widehat{d} \\
\widehat{\Theta} \\
\widehat{j_{0}} \\
\widehat{j_{1}}
\end{array}\right)=A(\xi)\left(\begin{array}{c}
\widehat{a} \\
\widehat{d} \\
\widehat{\Theta} \\
\widehat{j_{0}} \\
\widehat{j_{1}}
\end{array}\right),
$$


where, omitting the underlines from now on for better readability,

$$
A(\xi):=\left(\begin{array}{ccccc}
0 & -|\xi| & 0 & 0 & 0 \\
\frac{\alpha_{1}}{M a^{2}}|\xi| & -\frac{v}{\operatorname{Re}}|\xi|^{2} & \frac{\alpha_{2}}{M a^{2}}|\xi| & 0 & \mathcal{L} \beta \\
0 & -\alpha_{2}|\xi| & -\frac{\kappa}{\operatorname{Pr} R e}|\xi|^{2}-\frac{\mathcal{C L}}{\operatorname{Pr}} \sigma_{a} \alpha^{\prime} & \frac{\mathcal{C} \mathcal{L}}{\operatorname{Pr}} \sigma_{a} & 0 \\
0 & 0 & \mathcal{C} \mathcal{L} \sigma_{a} \alpha^{\prime} & -\mathcal{C} \mathcal{L} \sigma_{a} & -\frac{1}{n} \mathcal{C}|\xi| \\
0 & 0 & 0 & \mathcal{C}|\xi| & -n \mathcal{C} \mathcal{L} \beta
\end{array}\right)
$$

\subsection{The rescaled system in Fourier variables}

System (34) enters in the following class of linear systems:

$$
\left\{\begin{array}{l}
\partial_{t} a+\Lambda d=0 \\
\partial_{t} d-b_{1} \Lambda a-b_{1}^{\prime} \Lambda \Theta-b_{2} \Delta d-b_{3} j_{1}=0 \\
\partial_{t} \Theta+c_{1} \Lambda d+c_{2} \Theta-c_{3} \Delta \Theta-c_{4} j_{0}=0 \\
\partial_{t} j_{0}+b_{5} j_{0}+b_{6} \Lambda j_{1}-b_{4} \Theta=0 \\
\partial_{t} j_{1}+b_{8} j_{1}-b_{7} \Lambda j_{0}=0
\end{array}\right.
$$

The case we are interested in corresponds to

$b_{1}=\frac{\alpha_{1}}{M a^{2}}, \quad b_{2}=\frac{v}{R e}, \quad b_{1}^{\prime}=\frac{\alpha_{2}}{M a^{2}}, \quad b_{3}=\beta \mathcal{L}, \quad b_{4}=\mathcal{C} \mathcal{L} \sigma_{a} \alpha^{\prime}, \quad b_{5}=\mathcal{C} \mathcal{L} \sigma_{a}, \quad b_{6}=\frac{\mathcal{C}}{n}$, $b_{7}=\mathcal{C}, \quad b_{8}=n \beta \mathcal{C} \mathcal{L}, \quad c_{1}=\alpha_{2}, \quad c_{2}=\frac{\mathcal{C} \mathcal{L}}{\operatorname{Pr}} \sigma_{a} \alpha^{\prime}, \quad c_{3}=\frac{\kappa}{\operatorname{RePr}}, \quad c_{4}=\frac{\mathcal{C} \mathcal{L}}{\operatorname{Pr}} \sigma_{a}$.

In order to simplify the analysis, we shall first reduce the number of parameters in the above system by performing a convenient rescaling of the unknowns $a, d, \Theta, j_{0}, j_{1}$, and of the time and space variables. More precisely, setting

$$
\begin{aligned}
& a(t, x)=\widetilde{a}(\tau t, \chi x), \quad d(t, x)=\delta \widetilde{d}(\tau t, \chi x), \quad \Theta(t, x)=\delta^{\prime} \widetilde{\Theta}(\tau t, \chi x), \\
& j_{0}(t, x)=\zeta_{0} \widetilde{j_{0}}(\tau t, \chi x), \quad j_{1}(t, x)=\zeta_{1} \widetilde{j}_{1}(\tau t, \chi x),
\end{aligned}
$$

with

$$
\tau:=b_{1}, \quad \chi:=\sqrt{b_{1}}, \quad \delta:=\sqrt{b_{1}}, \quad \delta^{\prime}:=\frac{b_{1}}{b_{1}^{\prime}}, \quad \zeta_{0}:=\frac{b_{1}}{b_{3}} \sqrt{\frac{b_{6} b_{1}}{b_{7}}}, \quad \zeta_{1}:=\frac{b_{1}^{3 / 2}}{b_{3}},
$$

allows to reduce the number of parameters to nine. ${ }^{4}$ We eventually get the following system for $\left(\widetilde{a}, \widetilde{d}, \widetilde{\Theta}, \widetilde{j_{0}}, \widetilde{j_{1}}\right)$ :

$$
\left\{\begin{array}{l}
\partial_{t} \tilde{a}+\Lambda \widetilde{d}=0 \\
\partial_{t} \widetilde{d}-\Lambda \widetilde{a}-\Lambda \widetilde{\Theta}-\bar{v} \Delta \widetilde{d}-\widetilde{j_{1}}=0, \\
\partial_{t} \widetilde{\Theta}+\eta_{2} \Lambda \widetilde{d}+\eta_{3} \widetilde{\Theta}-\bar{\kappa} \Delta \widetilde{\Theta}-\eta_{4} \widetilde{j_{0}}=0 \\
\partial_{t} \widetilde{j_{0}}+\eta_{5} \widetilde{j_{0}}+\eta_{6} \Lambda \widetilde{j}_{j_{1}}-\eta_{7} \widetilde{\Theta}=0 \\
\partial_{t} \widetilde{j}_{1}+\eta_{8} \widetilde{j}_{1}-\eta_{6} \Lambda \widetilde{j_{0}}=0
\end{array}\right.
$$

with

$$
\left\{\begin{array}{l}
\bar{v}=\frac{\nu}{R e}, \bar{\kappa}=\frac{\kappa}{R e P r}, \quad \eta_{2}=\frac{c_{1} b_{1}^{\prime}}{b_{1}}=\frac{\alpha_{2}^{2}}{\alpha_{1}}, \quad \eta_{3}=\frac{c_{2}}{b_{1}}=\frac{\mathcal{C} \mathcal{L} \sigma_{a} M a^{2} \alpha^{\prime}}{\alpha_{1} P r}, \\
\eta_{4}=\frac{c_{4} b_{1}^{\prime}}{b_{3}} \sqrt{\frac{b_{6}}{b_{1} b_{7}}}=\frac{\mathcal{C} \sigma_{a} \alpha_{2}}{\sqrt{n \alpha_{1}} \beta P r M a}, \quad \eta_{5}=\frac{b_{5}}{b_{1}}=\frac{\mathcal{C} \mathcal{L} \sigma_{a} M a^{2}}{\alpha_{1}}, \quad \eta_{6}=\sqrt{\frac{b_{6} b_{7}}{b_{1}}=\frac{\mathcal{C} M a}{\sqrt{n \alpha_{1}}},} \\
\eta_{7}=\frac{b_{3} b_{4}}{b_{1} b_{1}^{\prime}} \sqrt{\frac{b_{7}}{b_{1} b_{6}}}=\frac{\sqrt{n} \mathcal{C} \mathcal{L}^{2} \beta \sigma_{a} \alpha^{\prime} M a^{5}}{\alpha_{1}^{3 / 2} \alpha_{2}}, \quad \eta_{8}=\frac{b_{8}}{b_{1}}=\frac{n \beta \mathcal{C} M a^{2}}{\alpha_{1}}
\end{array}\right.
$$

4 It would be possible to set in addition the viscosity or the conductivity to 1 . However we prefer to keep track of the two coefficients in the computations. 
where

$$
\beta=\frac{1}{n}\left(\sigma_{a}+\mathcal{L}_{s} \sigma_{s}\right), \quad \alpha_{1}=\partial_{\varrho} p(1,1), \quad \alpha_{2}=\partial_{\vartheta} p(1,1), \quad \alpha^{\prime}=\partial_{\vartheta} b(1)
$$

Let us point out that the coefficients $\eta_{3}, \eta_{4}, \eta_{5}$ and $\eta_{7}$ are interrelated through

$$
\eta_{3} \eta_{5}-\eta_{4} \eta_{7}=0
$$

This will be of importance in some of the computations that follow.

Setting $\rho:=|\xi|$, System (37) in Fourier variables reads (omitting tildes from now on)

$$
\frac{\mathrm{d}}{\mathrm{d} t}\left(\begin{array}{c}
\widehat{a} \\
\widehat{d} \\
\widehat{\Theta} \\
\widehat{j_{0}} \\
\widehat{j_{1}}
\end{array}\right)+\left(\begin{array}{ccccc}
0 & \rho & 0 & 0 & 0 \\
-\rho & \bar{v} \rho^{2} & -\rho & 0 & 1 \\
0 & \eta_{2} \rho & \eta_{3}+\bar{\kappa} \rho^{2} & -\eta_{4} & 0 \\
0 & 0 & -\eta_{7} & \eta_{5} & \eta_{6} \rho \\
0 & 0 & 0 & -\eta_{6} \rho & \eta_{8}
\end{array}\right)\left(\begin{array}{c}
\widehat{a} \\
\widehat{d} \\
\widehat{\Theta} \\
\widehat{j_{0}} \\
\widehat{j_{1}}
\end{array}\right)=\left(\begin{array}{l}
0 \\
0 \\
0 \\
0 \\
0
\end{array}\right),
$$

that is to say

$$
\partial_{t} \widehat{W}+A \widehat{W}+\rho B \widehat{W}+\rho^{2} C \widehat{W}=0
$$

where

$$
\begin{aligned}
\widehat{W}: & =\left(\begin{array}{c}
\widehat{a} \\
\widehat{d} \\
\widehat{\Theta} \\
\widehat{j_{0}} \\
\widehat{j_{1}}
\end{array}\right), \quad A:=\left(\begin{array}{ccccc}
0 & 0 & 0 & 0 & 0 \\
0 & 0 & 0 & 0 & -1 \\
0 & 0 & \eta_{3} & -\eta_{4} & 0 \\
0 & 0 & -\eta_{7} & \eta_{5} & 0 \\
0 & 0 & 0 & 0 & \eta_{8}
\end{array}\right), \\
B & :=\left(\begin{array}{ccccccccc}
0 & 1 & 0 & 0 & 0 \\
-1 & 0 & -1 & 0 & 0 \\
0 & \eta_{2} & 0 & 0 & 0 \\
0 & 0 & 0 & 0 & \eta_{6} \\
0 & 0 & 0 & -\eta_{6} & 0
\end{array}\right), C:=\left(\begin{array}{cccccc}
0 & 0 & 0 & 0 & 0 \\
0 & \bar{v} & 0 & 0 & 0 \\
0 & 0 & \bar{\kappa} & 0 & 0 \\
0 & 0 & 0 & 0 & 0 \\
0 & 0 & 0 & 0 & 0
\end{array}\right) .
\end{aligned}
$$

\subsection{Low-frequency decay estimates}

The starting point is the observation that, keeping (39) in mind, the eigenvalues of the matrix of System (40) for $\rho=0$ are 0 (with multiplicity 3), $\eta_{3}+\eta_{5}$ and $\eta_{8}$. The corresponding modes are

$$
\widehat{a}, \quad \widehat{d}+\frac{1}{\eta_{8}} \widehat{j_{1}}, \quad \widehat{\Theta}+\frac{\eta_{4}}{\eta_{5}} \widehat{j_{0}}, \quad \widehat{j_{0}}-\frac{\eta_{3}}{\eta_{4}} \widehat{\Theta}, \quad \widehat{j_{1}} .
$$

Changing unknowns accordingly, System (41) rewrites

$$
\partial_{t} \widehat{U}+A_{0} \widehat{U}+\rho B_{0} \widehat{U}+\rho^{2} C_{0} \widehat{U}=0 \quad \text { with } \quad \widehat{U}:=\left(\begin{array}{c}
\widehat{a} \\
\widehat{d}+\frac{1}{\eta_{8}} \widehat{j_{1}} \\
\widehat{\Theta}+\frac{\eta_{4}}{\eta_{5}} \widehat{j_{0}} \\
\widehat{j_{0}}-\frac{\eta_{3}}{\eta_{4}} \widehat{\Theta} \\
\hat{j}_{1}
\end{array}\right)=\Pi^{-1} \widehat{W}
$$


Remembering that $\eta_{3} \eta_{5}=\eta_{4} \eta_{7}$, we have

$$
\Pi=\left(\begin{array}{ccccc}
1 & 0 & 0 & 0 & 0 \\
0 & 1 & 0 & 0 & -\frac{1}{\eta_{8}} \\
0 & 0 & \widetilde{\eta}_{5} & -\widetilde{\eta}_{4} & 0 \\
0 & 0 & \widetilde{\eta}_{7} & \widetilde{\eta}_{5} & 0 \\
0 & 0 & 0 & 0 & 1
\end{array}\right) \quad \text { and } \quad \Pi^{-1}=\left(\begin{array}{ccccc}
1 & 0 & 0 & 0 & 0 \\
0 & 1 & 0 & 0 & \frac{1}{\eta_{8}} \\
0 & 0 & 1 & \frac{\eta_{4}}{\eta_{5}} & 0 \\
0 & 0 & -\frac{\eta_{3}}{\eta_{4}} & 1 & 0 \\
0 & 0 & 0 & 0 & 1
\end{array}\right),
$$

with the rescaled coefficients $\widetilde{\eta}_{i}(i \geq 3)$ defined by

$$
\tilde{\eta}_{i}:=\frac{\eta_{i}}{\eta_{3}+\eta_{5}} \text { for } i \in\{3,4,5,7\}, \text { and } \tilde{\eta}_{6}:=\frac{\eta_{6}}{\eta_{8}} .
$$

Note that the coefficients $\widetilde{\eta}_{i}$ are of order 1 and $\Pi$ is thus nicely conditioned in the asymptotics $\mathcal{C} \rightarrow+\infty$.

Let us compute the matrices $A_{0}, B_{0}$ and $C_{0}$. We have

$$
\begin{aligned}
& A_{0}=\Pi^{-1} A \Pi=\left(\begin{array}{ccccc}
0 & 0 & 0 & 0 & 0 \\
0 & 0 & 0 & 0 & 0 \\
0 & 0 & 0 & 0 & 0 \\
0 & 0 & 0 & \eta_{3}+\eta_{5} & 0 \\
0 & 0 & 0 & 0 & \eta_{8}
\end{array}\right), \\
& B_{0}=\Pi^{-1} B \Pi=\left(\begin{array}{ccccc}
0 & 1 & 0 & 0 & -\frac{1}{\eta_{8}} \\
-1 & 0 & -\tilde{\eta}_{5}-\tilde{\eta}_{6} \tilde{\eta}_{7} & \tilde{\eta}_{4}-\tilde{\eta}_{5} \tilde{\eta}_{6} & 0 \\
0 & \eta_{2} & 0 & 0 & \frac{\eta_{4} \eta_{6}}{\eta_{5}}-\frac{\eta_{2}}{\eta_{8}} \\
0 & -\frac{\eta_{2} \eta_{3}}{\eta_{4}} & 0 & 0 & \frac{\eta_{2} \eta_{3}}{\eta_{4} \eta_{8}}+\eta_{6} \\
0 & 0 & -\eta_{6} \tilde{\eta}_{7} & -\eta_{6} \tilde{\eta}_{5} & 0
\end{array}\right),
\end{aligned}
$$

and

$$
C_{0}=\Pi^{-1} C \Pi=\left(\begin{array}{ccccc}
0 & 0 & 0 & 0 & 0 \\
0 & \bar{v} & 0 & 0 & -\frac{\bar{v}}{\eta_{8}} \\
0 & 0 & \widetilde{\eta}_{5} \bar{\kappa} & -\widetilde{\eta}_{4} \bar{\kappa} & 0 \\
0 & 0 & -\widetilde{\eta}_{7} \bar{\kappa} & \widetilde{\eta}_{3} \bar{\kappa} & 0 \\
0 & 0 & 0 & 0 & 0
\end{array}\right)
$$

One splits $B_{0}$ into $A_{1}+B_{1}$, where

$$
A_{1}=\left(\begin{array}{ccccc}
0 & 1 & 0 & 0 & 0 \\
-1 & 0 & -\tilde{\eta}_{5}-\tilde{\eta}_{6} \tilde{\eta}_{7} & 0 & 0 \\
0 & \eta_{2} & 0 & 0 & \frac{\eta_{4} \eta_{6}}{\eta_{5}}-\frac{\eta_{2}}{\eta_{8}} \\
0 & 0 & 0 & 0 & \frac{\eta_{2} \eta_{3}}{\eta_{4} \eta_{8}}+\eta_{6} \\
0 & 0 & -\eta_{6} \tilde{\eta}_{7} & -\eta_{6} \tilde{\eta}_{5} & 0
\end{array}\right)
$$

and

$$
B_{1}=\left(\begin{array}{ccccc}
0 & 0 & 0 & 0 & -\frac{1}{\eta_{8}} \\
0 & 0 & 0 & \tilde{\eta}_{4}-\tilde{\eta}_{5} \tilde{\eta}_{6} & 0 \\
0 & 0 & 0 & 0 & 0 \\
0 & -\frac{\eta_{2} \eta_{3}}{\eta_{4}} & 0 & 0 & 0 \\
0 & 0 & 0 & 0 & 0
\end{array}\right)
$$

On the one hand, $A_{1}$ can be antisymmetrized by some positive diagonal matrix and is thus harmless from the point of view of decay estimates. On the other hand, $B_{1}$ does not have 
much structure and is likely to spoil our analysis as it cannot be completely counterbalanced by the matrix $A_{0}$ which is degenerate. As observed in $[13,14]$ in a simpler context, the bad contribution of $B_{1}$ may be somewhat weakened if performing a second change of unknowns

$$
V:=\left(\mathbb{I}_{d}+\rho P\right) \widehat{U}
$$

for a $5 \times 5$ matrix $P$ such that

$$
\left[P, A_{0}\right]+B_{1}=0 .
$$

Indeed setting $A_{3}:=\left(P A_{0}-A_{1}\right) P+C_{0}$, we get the identity

$$
\partial_{t} V+A_{0} V+\rho A_{1} V+\rho^{2}\left(C_{0}+P B_{1}+\left[P, A_{1}\right]\right) V=\rho^{3}\left[A_{3}, P\right]\left(\mathbb{I}_{d}+\rho P\right)^{-1} V .
$$

Let us rewrite the previous matrices in block form $M=\left(\begin{array}{ll}M^{11} & M^{12} \\ M^{21} & M^{22}\end{array}\right)$, where $M^{11}$ is a $3 \times 3$ block and $M^{22}$ is a $2 \times 2$ block:

$$
B_{1}=\left(\begin{array}{cc}
0 & B_{1}^{12} \\
B_{1}^{21} & 0
\end{array}\right), \quad A_{0}=\left(\begin{array}{cc}
0 & 0 \\
0 & \Delta
\end{array}\right), \quad A_{1}=\left(\begin{array}{cc}
A_{1}^{11} & 0 \\
0 & A_{1}^{22}
\end{array}\right), \quad P=\left(\begin{array}{ll}
P^{11} & P^{12} \\
P^{21} & P^{22}
\end{array}\right) .
$$

Hence

$$
\left[P, A_{0}\right]=\left(\begin{array}{cc}
0 & P^{12} \Delta \\
-\Delta P^{21} & {\left[P^{22}, \Delta\right]}
\end{array}\right)
$$

and one can thus ensure (43) if taking

$$
P^{11}=0, \quad P^{22}=0, \quad P^{12}=-B_{1}^{12} \Delta^{-1} \text { and } P^{21}=\Delta^{-1} B_{1}^{21},
$$

that is to say,

$$
P=\left(\begin{array}{ccccc}
0 & 0 & 0 & 0 & p_{1} \\
0 & 0 & 0 & p_{2} & 0 \\
0 & 0 & 0 & 0 & 0 \\
0 & p_{4} & 0 & 0 & 0 \\
0 & 0 & 0 & 0 & 0
\end{array}\right) \quad \text { with } \quad p_{1}:=\frac{1}{\eta_{8}^{2}}, \quad p_{2}:=\frac{\widetilde{\eta}_{5} \widetilde{\eta}_{6}-\widetilde{\eta}_{4}}{\eta_{3}+\eta_{5}} \text { and } p_{4}:=-\frac{\eta_{2} \widetilde{\eta}_{3}}{\eta_{4}} .
$$

So we end up with

$$
V=\left(\begin{array}{c}
\widehat{\mathfrak{a}} \\
\widehat{d} \\
\widehat{\Theta} \\
\widehat{\dot{j}_{0}} \\
\hat{j}_{1}
\end{array}\right)=\left(\mathbb{I}_{d}+\rho P\right) \Pi^{-1} \widehat{W}=\left(\begin{array}{ccccc}
1 & 0 & 0 & 0 & \rho p_{1} \\
0 & 1 & -\frac{\rho \eta_{3} p_{2}}{\eta_{4}} & \rho p_{2} & \frac{1}{\eta_{8}} \\
0 & 0 & 1 & \frac{\eta_{4}}{\eta_{5}} & 0 \\
0 & \rho p_{4} & -\frac{\eta_{3}}{\eta_{4}} & 1 & \frac{\rho p_{4}}{\eta_{8}} \\
0 & 0 & 0 & 0 & 1
\end{array}\right)\left(\begin{array}{c}
\widehat{a} \\
\widehat{d} \\
\widehat{\Theta} \\
\widehat{j_{0}} \\
\widehat{j_{1}}
\end{array}\right) .
$$

We notice that $\operatorname{det} \Pi^{-1}=1+\frac{\eta_{3}}{\eta_{5}}$ and that

$$
\operatorname{det}\left(\mathbb{I}_{d}+\rho P\right)=\operatorname{det}\left(\begin{array}{ccccc}
1 & 0 & 0 & 0 & \rho p_{1} \\
0 & 1 & 0 & \rho p_{2} & 0 \\
0 & 0 & 1 & 0 & 0 \\
0 & \rho p_{4} & 0 & 1 & 0 \\
0 & 0 & 0 & 0 & 1
\end{array}\right)=1-\rho^{2} p_{2} p_{4}
$$

Given that $\eta_{3} / \eta_{5}$ is of order 1 and that all the $p_{i}$ 's are of order at most $\mathcal{C}^{-1}$, we deduce that

$$
|\widehat{W}| \approx|V| \text { whenever } \rho \ll \mathcal{C} .
$$


Let us also emphasize that all the coefficients of $C_{0}$ are of order at most 1 , and that the coefficients of $A_{0}$ and of $A_{1}$ are of order at most $\mathcal{C}$. Therefore the matrix $\left(P A_{0}-A_{1}\right) P+C_{0}$ is $\mathcal{O}(1)$ and the commutator $\left[A_{3}, P\right]$ in (44) is thus of order $\mathcal{C}^{-1}$. So finally, one can write that if $\rho \ll \mathcal{C}$ then

$$
\frac{\mathrm{d}}{\mathrm{d} t} V+A_{0} V+\rho A_{1} V+\rho^{2} B_{2} V=\mathcal{O}\left(\mathcal{C}^{-1} \rho^{3}\right) \quad \text { with } B_{2}:=C_{0}+P B_{1}+\left[P, A_{1}\right] .
$$

To go further into our analysis, computing $P B_{1}$ and $\left[P, A_{1}\right]$ is required. We find that

$$
\begin{aligned}
& P B_{1}=\left(\begin{array}{ccccc}
0 & 0 & 0 & 0 & 0 \\
0 & -\gamma & 0 & 0 & 0 \\
0 & 0 & 0 & 0 & 0 \\
0 & 0 & 0 & \gamma & 0 \\
0 & 0 & 0 & 0 & 0
\end{array}\right) \text { with } \gamma:=\frac{\eta_{2} \widetilde{\eta}_{3}}{\eta_{4}}\left(\widetilde{\eta}_{5} \widetilde{\eta}_{6}-\widetilde{\eta}_{4}\right)=\frac{\eta_{2}}{\eta_{3}+\eta_{5}}\left(\widetilde{\eta}_{6} \widetilde{\eta}_{7}-\widetilde{\eta}_{3}\right) \text {, } \\
& P A_{1}=\left(\begin{array}{ccccc}
0 & 0 & -\frac{\tilde{\eta}_{6} \widetilde{\eta}_{7}}{\eta_{8}} & -\frac{\tilde{\eta}_{6} \widetilde{\eta}_{5}}{\eta_{8}} & 0 \\
0 & 0 & 0 & 0 & \eta_{6}\left(1+\frac{\eta_{2} \eta_{3}}{\eta_{4} \eta_{6} \eta_{8}}\right)\left(\frac{\tilde{\eta}_{5} \widetilde{\eta}_{6}-\widetilde{\eta}_{4}}{\eta_{3}+\eta_{5}}\right) \\
0 & 0 & 0 & 0 & 0 \\
\frac{\eta_{2} \widetilde{\eta}_{3}}{\eta_{4}} & 0 & \frac{\eta_{2} \widetilde{\eta}_{3}}{\eta_{4}}\left(\widetilde{\eta}_{5}+\widetilde{\eta}_{6} \widetilde{\eta}_{7}\right) & 0 & 0 \\
0 & 0 & 0 & 0 & 0
\end{array}\right)
\end{aligned}
$$

and

$$
A_{1} P=\left(\begin{array}{ccccc}
0 & 0 & 0 & \frac{\tilde{\eta}_{5} \widetilde{\eta}_{6}-\widetilde{\eta}_{4}}{\eta_{3}+\eta_{5}} & 0 \\
0 & 0 & 0 & 0 & -\frac{1}{\eta_{8}^{2}} \\
0 & 0 & 0 & \eta_{2}\left(\frac{\widetilde{\eta}_{5} \widetilde{\eta}_{6}-\widetilde{\eta}_{4}}{\eta_{3}+\eta_{5}}\right) & 0 \\
0 & 0 & 0 & 0 & 0 \\
0 & \frac{\eta_{2} \widetilde{\eta}_{3} \widetilde{\eta}_{5} \eta_{6}}{\eta_{4}} & 0 & 0 & 0
\end{array}\right) .
$$

Therefore $B_{2}=\left(\begin{array}{ll}B_{2}^{11} & B_{2}^{12} \\ B_{2}^{21} & B_{2}^{22}\end{array}\right)$ with

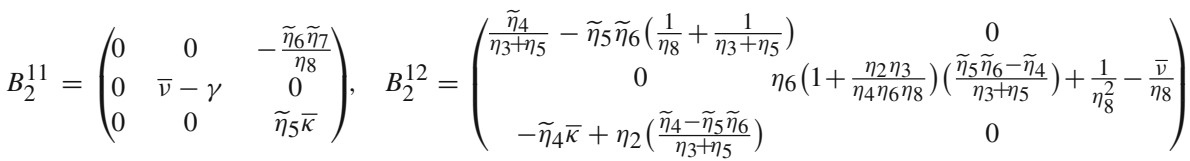

$$
\begin{aligned}
& B_{2}^{21}=\left(\begin{array}{ccc}
\frac{\eta_{2} \widetilde{\eta}_{3}}{\eta_{4}} & 0 & \frac{\eta_{2} \widetilde{\eta}_{3}}{\eta_{4}}\left(\widetilde{\eta}_{5}+\widetilde{\eta}_{6} \tilde{\eta}_{7}\right)-\widetilde{\eta}_{7} \bar{\kappa} \\
0 & -\frac{\eta_{2} \tilde{\eta}_{3} \tilde{\eta}_{5} \eta_{6}}{\eta_{4}} & 0
\end{array}\right), \quad B_{2}^{22}=\left(\begin{array}{ccc}
\gamma+\widetilde{\eta}_{3} \bar{\kappa} & 0 \\
0 & 0
\end{array}\right) .
\end{aligned}
$$

Let us observe that $A_{0}$ is a nonnegative (degenerate) diagonal matrix of order $\mathcal{C}$, that $A_{1}$ is also of order $\mathcal{C}$ but anti-symmetrizable through a diagonal matrix of order 1 , and is thus likely to have no influence in the energy-type estimates. The leading order terms of $B_{2}$ are of order 1. They are located either on the diagonal (and are positive if $\bar{v}>\gamma>0$ and $\bar{\kappa}>0$ ) or in the blocks $B_{2}^{12}$ and $B_{2}^{21}$ that correspond to interactions between the (modified) fluid unknowns ${ }^{t} V_{1}:=(\widehat{\mathfrak{a}}, \widehat{\mathfrak{d}}, \widehat{\Theta})$ and radiative unknowns ${ }^{t} V_{2}:=\left(\widehat{j_{0}}, \widehat{j_{1}}\right)$. Therefore an important part of the stability analysis will be dedicated to the $3 \times 3$ subsystem with matrix $A_{1}^{11} \rho+B_{2}^{11} \rho^{2}$ satisfied by $V_{1}$, and to the $2 \times 2$ subsystem with matrix $\Delta+\rho A_{1}^{22}+\rho^{2} B_{2}^{22}$ fulfilled by $V_{2}$. For both sub-systems, interactions between the fluid unknowns $V_{1}$ and radiative unknowns $V_{2}$ will be considered as error terms in the right-hand side, that may be eliminated for small enough $\rho$. More concretely: 


$$
\partial_{t} V_{1}+\rho A_{1}^{11} V_{1}+\rho^{2} B_{2}^{11} V_{1}=-\rho^{2} B_{2}^{12} V_{2}+\mathcal{O}\left(\mathcal{C}^{-1} \rho^{3}\right)
$$

and

$$
\partial_{t} V_{2}+\left(\Delta+\rho^{2} B_{2}^{22}\right) V_{2}+\rho A_{1}^{22} V_{2}=-\rho^{2} B_{2}^{21} V_{1}+\mathcal{O}\left(\mathcal{C}^{-1} \rho^{3}\right) .
$$

Let us first investigate the system fulfilled by the (modified) hydrodynamic unknowns $V_{1}$, looking at the coupling with $V_{2}$ as a source term. Denoting

$$
v:=\bar{v}-\gamma, \quad \kappa:=\widetilde{\eta}_{5} \bar{\kappa}, \quad \varepsilon:=\frac{\tilde{\eta}_{6} \tilde{\eta}_{7}}{\eta_{8}}, \quad \widetilde{\alpha}:=\widetilde{\eta}_{5}+\tilde{\eta}_{6} \tilde{\eta}_{7} \text { and } \alpha:=\eta_{2},
$$

that system reads

$$
\frac{\mathrm{d}}{\mathrm{d} t}\left(\begin{array}{l}
\widehat{\mathfrak{a}} \\
\widehat{\mathfrak{d}} \\
\widehat{\mathfrak{t}}
\end{array}\right)+\rho\left(\begin{array}{ccc}
0 & 1 & 0 \\
-1 & 0 & -\widetilde{\alpha} \\
0 & \alpha & 0
\end{array}\right)\left(\begin{array}{l}
\widehat{\mathfrak{a}} \\
\widehat{\mathfrak{d}} \\
\widehat{\mathfrak{t}}
\end{array}\right)+\rho^{2}\left(\begin{array}{ccc}
0 & 0 & -\varepsilon \\
0 & \nu & 0 \\
0 & 0 & \kappa
\end{array}\right)\left(\begin{array}{l}
\widehat{\mathfrak{a}} \\
\widehat{\mathfrak{d}} \\
\widehat{\mathfrak{t}}
\end{array}\right)=\left(\begin{array}{c}
\widehat{f} \\
\widehat{g} \\
\widehat{h}
\end{array}\right) .
$$

The associated characteristic polynomial reads

$$
P_{\rho}(\lambda)=-\lambda^{3}+a_{1}(\rho) \lambda^{2}-a_{2}(\rho) \lambda+a_{3}(\rho)
$$

with $a_{1}(\rho)=(v+\kappa) \rho, a_{2}(\rho)=1+\alpha \widetilde{\alpha}+v \kappa \rho^{2}$ and $a_{3}(\rho)=(\kappa+\alpha \varepsilon) \rho$.

Note that (49) ensures that the coefficients $\kappa, \varepsilon, \alpha$ and $\widetilde{\alpha}$ are positive. As regards $v$, a necessary condition for all the real parts of the roots of the polynomial $P_{\rho}$ to be positive is that $a_{1}(\rho) \geq 0$, and thus $v+\kappa \geq 0$. Now if that latter condition is fulfilled then LiénardChipart criterion [21] guarantees that the real parts of all the roots of the polynomial $P_{\rho}$ $(\rho>0)$ are positive if and only if

$$
a_{1}(\rho) a_{2}(\rho)-a_{3}(\rho)=((\nu+\kappa)(1+\alpha \widetilde{\alpha})-\kappa-\alpha \varepsilon) \rho+v \kappa(\nu+\kappa) \rho^{2}>0 .
$$

We thus find out the following necessary and sufficient stability condition for (50):

$$
\varepsilon<\widetilde{\alpha} \kappa+\left(\frac{1}{\alpha}+\widetilde{\alpha}\right) v \text { and } v+\kappa>0 .
$$

In terms of the coefficients of System (37), Condition (51) reads

$$
\begin{aligned}
& \left(\frac{\eta_{5} \eta_{8}+\eta_{6} \eta_{7}}{\eta_{3}+\eta_{5}}\right)\left(\left(\eta_{3}+\eta_{5}\right) \bar{v}+\eta_{5} \bar{\kappa}-\frac{\eta_{2}}{\eta_{3}+\eta_{5}}\left(\frac{\eta_{6} \eta_{7}}{\eta_{8}}-\eta_{3}\right)\right)+\frac{\eta_{8}}{\eta_{2}}\left(\eta_{3}+\eta_{5}\right) \bar{v} \\
& \quad+\frac{\eta_{3} \eta_{8}}{\eta_{3}+\eta_{5}}>\eta_{6} \eta_{7}\left(\frac{1}{\eta_{8}}+\frac{1}{\eta_{3}+\eta_{5}}\right) \text { and }\left(\eta_{3}+\eta_{5}\right) \bar{v}+\eta_{5} \bar{\kappa}>\left(\frac{\eta_{2}}{\eta_{3}+\eta_{5}}\right)\left(\frac{\eta_{6} \eta_{7}}{\eta_{8}}-\eta_{3}\right) .
\end{aligned}
$$

Resuming to the definition of coefficients $\eta_{i}$, we find (31). In particular, this implies that (51) is satisfied for $\mathcal{C} \rightarrow+\infty$.

We aim at recovering that stability condition, supplemented with explicit decay estimates in terms of $\rho$. We claim that if $\widehat{f}=\widehat{g}=\widehat{h} \equiv 0$ and (51) is fulfilled then there exists some positive threshold $\rho_{0}$, and two positive constants $c$ and $C$ (depending continuously on $\varepsilon, \alpha$, $\widetilde{\alpha}, \kappa$ and $\nu$ ) so that

$$
|(\widehat{\mathfrak{a}}, \widehat{\mathfrak{d}}, \widehat{\mathfrak{t}})(t)| \leq C e^{-c \rho^{2} t}|(\widehat{\mathfrak{a}}, \widehat{\mathfrak{d}}, \widehat{\mathfrak{t}})(0)| \text { for all } t>0 \text { and } \rho \in\left[0, \rho_{0}\right] .
$$

To prove our claim, let us introduce:

$$
\mathcal{L}_{\varepsilon_{1}, \varepsilon_{2}}^{2}(\rho):=|(\widehat{\mathfrak{a}}, \widehat{\mathfrak{d}})|^{2}+\frac{\widetilde{\alpha}}{\alpha}|\widehat{\mathfrak{t}}|^{2}-2 \varepsilon_{1} \rho \Re(\widehat{\mathfrak{a}} \widehat{\mathfrak{d}})+2 \varepsilon_{2} \rho \Re(\widehat{\mathfrak{d}} \overline{\mathfrak{t}}),
$$

for suitable parameters $\varepsilon_{1}>0$ and $\varepsilon_{2}>0$ to be chosen hereafter. 
From (50), we readily get

$$
\begin{aligned}
& \frac{1}{2} \frac{\mathrm{d}}{\mathrm{d} t}\left(|(\widehat{\mathfrak{a}}, \widehat{\mathfrak{d}})|^{2}+\frac{\widetilde{\alpha}}{\alpha} \mid \mathfrak{t}^{2}\right)+\left.v \rho^{2} \widehat{\mathfrak{d}}\right|^{2}+\kappa \frac{\widetilde{\alpha}}{\alpha} \rho^{2}|\widehat{\mathfrak{t}}|^{2}-\varepsilon \rho^{2} \Re(\widehat{\mathfrak{a}} \overline{\mathfrak{t}})=0, \\
& \frac{\mathrm{d}}{\mathrm{d} t} \Re(\widehat{\mathfrak{d}} \overline{\mathfrak{a}})+\rho|\widehat{\mathfrak{d}}|^{2}-\rho|\widehat{\mathfrak{a}}|^{2}-\widetilde{\alpha} \rho \Re(\widehat{\mathfrak{t}} \widehat{\mathfrak{a}})=\rho^{2} \Re((\widehat{\varepsilon} \widehat{\mathfrak{t}}-v \widehat{\mathfrak{a}}) \overline{\mathfrak{d}}), \\
& \frac{\mathrm{d}}{\mathrm{d} t} \Re(\widehat{\mathfrak{d}} \overline{\mathfrak{t}})+\alpha \rho|\widehat{\mathfrak{d}}|^{2}-\widetilde{\alpha} \rho \mid \widehat{\mathfrak{t}}^{2}-\rho \Re(\widehat{\mathfrak{a}} \overline{\mathfrak{t}})=-(v+\kappa) \rho^{2} \Re(\widehat{\mathfrak{d}} \widehat{\mathfrak{t}}) .
\end{aligned}
$$

Hence for all small enough $\rho$,

$$
\frac{1}{2} \frac{\mathrm{d}}{\mathrm{d} t} \mathcal{L}_{\varepsilon_{1}, \varepsilon_{2}}^{2}+\rho^{2} \mathcal{H}_{\varepsilon_{1}, \varepsilon_{2}}^{2}=\mathcal{O}\left(\rho^{3}\right)
$$

with

$$
\mathcal{H}_{\varepsilon_{1}, \varepsilon_{2}}^{2}(\widehat{\mathfrak{a}}, \widehat{\mathfrak{d}}, \widehat{\mathfrak{t}})=\varepsilon_{1}|\widehat{\mathfrak{a}}|^{2}+\left(v-\varepsilon_{1}+\alpha \varepsilon_{2}\right)|\widehat{\mathfrak{d}}|^{2}+\widetilde{\alpha}\left(\frac{\kappa}{\alpha}-\varepsilon_{2}\right) \mid \widehat{\mathfrak{t}}^{2}+\left(\widetilde{\alpha} \varepsilon_{1}-\varepsilon-\varepsilon_{2}\right) \Re(\widehat{\mathfrak{a}} \widehat{\mathfrak{t}}) .
$$

Note that we have

$$
\begin{aligned}
\mathcal{H}_{\varepsilon_{1}, \varepsilon_{2}}^{2}(\widehat{\mathfrak{a}}, \widehat{\mathfrak{d}}, \widehat{\mathfrak{t}})= & \varepsilon_{1}\left|\widehat{\mathfrak{a}}+\left(\frac{\widetilde{\alpha} \varepsilon_{1}-\varepsilon-\varepsilon_{2}}{2 \varepsilon_{1}}\right) \widehat{\mathfrak{t}}\right|^{2}+\left(v-\varepsilon_{1}+\alpha \varepsilon_{2}\right)|\widehat{\mathfrak{d}}|^{2} \\
& +\left.\widetilde{\alpha}\left(\frac{\kappa}{\alpha}-\varepsilon_{2}-\frac{\left(\widetilde{\alpha} \varepsilon_{1}-\varepsilon-\varepsilon_{2}\right)^{2}}{4 \widetilde{\alpha} \varepsilon_{1}}\right) \widehat{\mid \mathfrak{t}}\right|^{2} .
\end{aligned}
$$

Therefore $\mathcal{H}_{\varepsilon_{1}, \varepsilon_{2}}^{2}$ is a positive definite quadratic form if and only if

$$
\varepsilon_{1}>0, \quad \varepsilon_{1}<v+\alpha \varepsilon_{2} \text { and }\left(\widetilde{\alpha} \varepsilon_{1}-\varepsilon-\varepsilon_{2}\right)^{2}<4 \widetilde{\alpha} \varepsilon_{1}\left(\frac{\kappa}{\alpha}-\varepsilon_{2}\right) .
$$

We claim that if (51) is fulfilled then one can always find some $\varepsilon_{1}$ and $\varepsilon_{2}$ fulfilling (55).

In order to justify our claim, it is convenient to change $\left(\varepsilon_{1}, \varepsilon_{2}, \varepsilon\right)$ into $\left(\widetilde{\varepsilon}_{1}, \widetilde{\varepsilon}_{2}, \widetilde{\varepsilon}\right)$ as follows:

$\widetilde{\varepsilon}_{1}:=\frac{\widetilde{\alpha} \varepsilon_{1}}{\widetilde{\varepsilon}_{\max }}, \quad \widetilde{\varepsilon}_{2}:=\frac{1}{\widetilde{\varepsilon}_{\max }}\left(\frac{\kappa}{\alpha}-\varepsilon_{2}\right), \quad \widetilde{\varepsilon}:=\frac{1}{\widetilde{\varepsilon}_{\max }}\left(\varepsilon+\frac{\kappa}{\alpha}\right)$ with $\widetilde{\varepsilon}_{\max }:=\left(\frac{1}{\alpha}+\widetilde{\alpha}\right)(\nu+\kappa)$,

and, assuming that $v+\kappa>0$, to set

$$
\varepsilon_{1}^{\prime}:=\frac{\widetilde{\varepsilon}_{1}}{\widetilde{\varepsilon}} \text { and } \varepsilon_{2}^{\prime}:=\frac{\widetilde{\varepsilon}_{2}}{\widetilde{\varepsilon}} .
$$

Then, denoting $A:=\alpha \widetilde{\alpha}$, Condition (55) translates into

$$
\varepsilon_{1}^{\prime}>0, \quad \varepsilon_{1}^{\prime}+A \varepsilon_{2}^{\prime}<\frac{1}{\widetilde{\varepsilon}} \frac{A}{1+A} \quad \text { and } \quad\left(\varepsilon_{1}^{\prime}+\varepsilon_{2}^{\prime}-1\right)^{2}<4 \varepsilon_{1}^{\prime} \varepsilon_{2}^{\prime} .
$$

The latter condition is equivalent to

$$
L\left(\varepsilon_{1}^{\prime}, \varepsilon_{2}^{\prime}\right):=\left(\varepsilon_{1}^{\prime}-\varepsilon_{2}^{\prime}\right)^{2}-2\left(\varepsilon_{1}^{\prime}+\varepsilon_{2}^{\prime}\right)+1<0 .
$$

It is obvious that $L$ does not have any minimum in the interior of the domain $D$ defined by the first two conditions in (56), and that $L \geq 0$ for $\varepsilon_{1}^{\prime}=0$. For $\varepsilon_{2}^{\prime}=\widetilde{\varepsilon}^{-1} /(1+A)-\varepsilon_{1}^{\prime} / A$, we have

$$
L\left(\varepsilon_{1}^{\prime}, \varepsilon_{2}^{\prime}\right)=\left(\frac{A+1}{A} \varepsilon_{1}^{\prime}-\frac{\widetilde{\varepsilon}^{-1}}{A+1}\right)^{2}-2\left(\frac{A-1}{A} \varepsilon_{1}^{\prime}+\frac{\widetilde{\varepsilon}^{-1}}{A+1}\right)+1,
$$


the minimum of which corresponds to $\left(\varepsilon_{1}^{\prime}, \varepsilon_{2}^{\prime}\right)=\left(\varepsilon_{1}^{*}, \varepsilon_{2}^{*}\right)$ with

$$
\varepsilon_{1}^{*}=\frac{A\left(\widetilde{\varepsilon}^{-1}+A-1\right)}{(A+1)^{2}} \text { and } \varepsilon_{2}^{*}=\frac{A \widetilde{\varepsilon}^{-1}-A+1}{(A+1)^{2}} .
$$

The value of $L$ at $\left(\varepsilon_{1}^{*}, \varepsilon_{2}^{*}\right)$ is

$$
L\left(\varepsilon_{1}^{*}, \varepsilon_{2}^{*}\right)=\frac{4 A}{(A+1)^{2}}\left(1-\widetilde{\varepsilon}^{-1}\right) .
$$

Hence there exists $\left(\varepsilon_{1}^{*}, \varepsilon_{2}^{*}\right) \in D$ satisfying $L\left(\varepsilon_{1}^{*}, \varepsilon_{2}^{*}\right)<0$ if and only if $\widetilde{\varepsilon}^{-1}>1$, which is equivalent to the first part of the stability condition (51).

Let us recap. On the one hand, resuming to the initial parameters, we thus found some $\varepsilon_{1}$ and $\varepsilon_{2}$ satisfying (55). Taking such $\varepsilon_{1}$ and $\varepsilon_{2}$, the quadratic form $\mathcal{H}_{\varepsilon_{1}, \varepsilon_{2}}^{2}$ is definite positive, and thus $\left.\mathcal{H}_{\varepsilon_{1}, \varepsilon_{2}}^{2} \approx \mid \widehat{\mathfrak{a}}, \widehat{\mathfrak{d}}, \widehat{\mathfrak{t}}\right)\left.\right|^{2}$. On the other hand, for small enough $\rho$, we have

$$
\mathcal{L}_{\varepsilon_{1}, \varepsilon_{2}}^{2} \approx|(\widehat{\mathfrak{a}}, \widehat{\mathfrak{d}}, \widehat{\mathfrak{t}})|^{2} .
$$

Therefore (54) implies that there exists some $\rho_{0}>0$ and a constant $c>0$ depending only on the parameters of the system and such that

$$
\frac{\mathrm{d}}{\mathrm{d} t} \mathcal{L}_{\varepsilon_{1}, \varepsilon_{2}}^{2}+c \rho^{2} \mathcal{L}_{\varepsilon_{1}, \varepsilon_{2}}^{2} \leq 0 \text { if } 0 \leq \rho \leq \rho_{0} .
$$

This clearly implies (53). Now for general source terms $\widehat{f}, \widehat{g}$ and $\widehat{h}$ in (50), taking advantage of Duhamel's formula and integrating with respect to time gives for some $K=K(\varepsilon, \alpha, \widetilde{\alpha}, \kappa, v)$,

$$
|(\widehat{\mathfrak{a}}, \widehat{\mathfrak{d}}, \widehat{\mathfrak{t}})(t)|+c \rho^{2} \int_{0}^{t}|(\widehat{\mathfrak{a}}, \widehat{\mathfrak{d}}, \widehat{\mathfrak{t}})| \mathrm{d} \tau \leq K\left(|(\widehat{\mathfrak{a}}, \widehat{\mathfrak{d}}, \widehat{\mathfrak{t}})(0)|+\int_{0}^{t}|(\widehat{f}, \widehat{g}, \widehat{h})| \mathrm{d} \tau\right) .
$$

In the case we are interested in, the source terms $\widehat{f}, \widehat{g}$ and $\widehat{h}$ are given by the right-hand side of (47) and we thus have for small enough $\rho,|(\widehat{f}, \widehat{g}, \widehat{h})| \leq C \rho^{2}\left|V_{2}\right|+C \rho^{3}|V|$. Hence we deduce from (58) that

$$
\left|V_{1}(t)\right|+c \rho^{2} \int_{0}^{t}\left|V_{1}\right| \mathrm{d} \tau \leq K V_{1}(0)+K \rho^{2} \int_{0}^{t}\left|V_{2}\right| \mathrm{d} \tau .
$$

Handling the (modified) radiative unknowns $\left.\widehat{\hat{j}_{0}}, \widehat{j_{1}}\right)$ is much simpler. Indeed denoting $\varsigma:=1+$ $\frac{\eta_{2} \eta_{3}}{\eta_{4} \eta_{6} \eta_{8}}$, we have

$$
\frac{\mathrm{d}}{\mathrm{d} t}\left(\begin{array}{c}
\hat{\mathrm{j}}_{0} \\
\hat{\mathrm{j}}_{1}
\end{array}\right)+\left(\begin{array}{cc}
\eta_{3}+\eta_{5}+\rho^{2}\left(\gamma+\widetilde{\eta}_{3} \bar{\kappa}\right) & 0 \\
0 & \eta_{8}
\end{array}\right)\left(\begin{array}{c}
\hat{\mathrm{j}}_{0} \\
\hat{\mathrm{j}}_{1}
\end{array}\right)+\rho \eta_{6}\left(\begin{array}{cc}
0 & \varsigma \\
-1 & 0
\end{array}\right)\left(\begin{array}{l}
\hat{j_{0}} \\
\hat{\mathrm{j}}_{1}
\end{array}\right)=\left(\begin{array}{l}
\widehat{k} \\
\widehat{\ell}
\end{array}\right) .
$$

Therefore multiplying the second equation by $\varsigma$ and taking the inner product in $\mathbb{C}^{2}$ with $\left.\widehat{\left(j_{0}\right.}, \widehat{j}_{1}\right)$, we easily find that

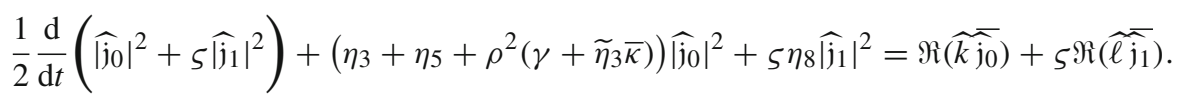

Hence

$$
\begin{aligned}
& \sqrt{\left.\widehat{\mid j_{0}}(t)\right|^{2}+\varsigma\left|\widehat{j}_{1}(t)\right|^{2}}+\min \left(\eta_{3}+\eta_{5}, \eta_{8}\right) \int_{0}^{t} \sqrt{\left|\widehat{j}_{0}\right|^{2}+\varsigma\left|\widehat{j_{1}}\right|^{2}} \mathrm{~d} \tau \\
& \leq \sqrt{\left.\widehat{\hat{j}_{0}}(0)\right|^{2}+\left.\varsigma \widehat{\mid}_{1}(0)\right|^{2}}+\int_{0}^{t} \sqrt{|\widehat{k}|^{2}+\varsigma|\widehat{\ell}|^{2}} \mathrm{~d} \tau .
\end{aligned}
$$


Because $\widehat{k}$ and $\widehat{\ell}$ are given by the right-hand side of (48), we have

$$
|(\widehat{k}, \widehat{\ell})| \leq C \rho^{2}\left|V_{1}\right|+C \rho^{3}|V|,
$$

and thus, for small enough $\rho$,

$$
\left|V_{2}(t)\right|+\int_{0}^{t}\left|V_{2}\right| \mathrm{d} \tau \leq C V_{2}(0)+C \rho^{2} \int_{0}^{t}\left|V_{1}\right| \mathrm{d} \tau .
$$

Putting that later inequality together with (59), we conclude that there exists $\rho_{0}>0$ depending only on the coefficients of System (40) such that if Condition (51) is fulfilled and $\rho \leq \rho_{0}$, then we have for all $t \geq 0$,

$$
|V(t)|+\rho^{2} \int_{0}^{t}\left|V_{1}\right| \mathrm{d} \tau+\int_{0}^{t}\left|V_{2}\right| \mathrm{d} \tau \leq C|V(0)| .
$$

In particular, this means that (51) is also a necessary and sufficient stability condition for the whole system (40), and that (61) holds provided $\rho \leq \rho_{0}$.

As we are interested in the asymptotics $\mathcal{C} \rightarrow+\infty$ and as $\varepsilon \approx \mathcal{C}^{-1}$, it is suitable to check what kind of information is supplied by the above analysis if $\varepsilon$ is small. On the one hand, in that case, the range for which (57) holds true is decreasing with respect to $\varepsilon_{1}$ and $\varepsilon_{2}$, which suggest us to take $\varepsilon_{1}$ and $\varepsilon_{2}$ as small as possible. On the other hand, the constant $c$ in (57) is of order $\varepsilon_{1}$, hence the decay becomes worse if taking $\varepsilon_{1}$ smaller. Therefore we need to find some acceptable compromise between having a large range of $\rho$ 's in (57) and a good decay.

By looking at Condition (55), we discover that whenever $\varepsilon<\widetilde{\alpha} \nu$, one can take $\varepsilon_{1}=\varepsilon / \widetilde{\alpha}$ and $\varepsilon_{2}=0$. As the error term in (54) is equal to $\varepsilon_{1} \rho^{3} \Re((\nu \widehat{\mathfrak{a}}-\varepsilon \widehat{\varepsilon}) \widehat{d})$, this gives for some constants $c$ and $\rho_{0}$ independent of $\varepsilon$,

$$
\frac{\mathrm{d}}{\mathrm{d} t} \mathcal{L}_{\rho}^{2}+c \varepsilon \rho^{2} \mathcal{L}_{\rho}^{2} \leq 0 \text { if } 0 \leq \rho \leq \rho_{0} \varepsilon^{-1 / 2}
$$

from which we get in particular,

$$
\forall t \geq 0, \varepsilon \rho^{2} \int_{0}^{t} \widehat{\mathfrak{t}}|\mathrm{d} \tau \leq C|(\mathfrak{a}, \mathfrak{d}, \mathfrak{t})(0) \mid .
$$

In order to improve the decay information that we have on $\widehat{\mathfrak{d}}$ and $\widehat{\mathfrak{t}}$ [which will be of fundamental importance in our study of the nonlinear system (25)], let us focus on the following linearized Navier-Stokes-Fourier system:

$$
\left\{\begin{array}{l}
\frac{\mathrm{d}}{\mathrm{d} d} \widehat{\mathfrak{a}}+\rho \widehat{\mathfrak{d}}=\widehat{k}:=\varepsilon \rho^{2} \widehat{\mathfrak{t}}, \\
\frac{\mathrm{d}}{\mathrm{d} t}+v \rho^{2} \widehat{\mathfrak{d}}-\rho \widehat{\mathfrak{a}}-\widetilde{\alpha} \widehat{\rho} \widehat{\mathfrak{t}}=0, \\
\frac{\mathrm{d}}{\mathrm{d} t} \widehat{\mathfrak{t}}+\kappa \rho^{2} \widehat{\mathfrak{t}}+\alpha \rho \widehat{\mathfrak{d}}=0 .
\end{array}\right.
$$

Assuming that $\widehat{k}=0$ for a while, we have:

$$
\begin{gathered}
\frac{1}{2} \frac{\mathrm{d}}{\mathrm{d} t}|(\widehat{\mathfrak{a}}, \widehat{\mathfrak{d}})|^{2}+v \rho^{2}|\widehat{\mathfrak{d}}|^{2}-\widetilde{\alpha} \rho \Re(\widehat{\mathfrak{t} \widehat{d}})=0, \\
\left.\left.\frac{1}{2} \frac{\mathrm{d}}{\mathrm{d} t} \widehat{\mid \mathfrak{t}}\right|^{2}+\kappa \rho^{2}|\widehat{\mathfrak{t}}|^{2}+\alpha \rho \Re \widehat{\mathfrak{t}} \widehat{\mathfrak{d}}\right)=0 .
\end{gathered}
$$

Therefore

$$
\frac{1}{2} \frac{\mathrm{d}}{\mathrm{d} t}\left(\alpha|(\widehat{\mathfrak{a}}, \widehat{\mathfrak{d}})|^{2}+\widetilde{\alpha}|\widehat{\mathfrak{t}}|^{2}\right)+\alpha v \rho^{2}|\widehat{\mathfrak{d}}|^{2}+\widetilde{\alpha} \kappa \rho^{2}|\widehat{\mathfrak{t}}|^{2}=0
$$


In addition,

$$
\frac{1}{2} \frac{\mathrm{d}}{\mathrm{d} t}|v \rho \widehat{a}|^{2}+v \rho^{2} \Re(v \rho \widehat{a} \widehat{\mathfrak{d}})=0
$$

and

$$
\frac{\mathrm{d}}{\mathrm{d} t} \Re(v \rho \widehat{\mathfrak{a}} \widehat{\mathfrak{d}})-v \rho^{2}|\widehat{\mathfrak{a}}|^{2}+v \rho^{2}|\widehat{\mathfrak{d}}|^{2}-\widetilde{\alpha} \rho^{2} v \Re(\widehat{\mathfrak{t}} \widehat{\mathfrak{a}})+v \rho^{2} \Re(v \rho \widehat{a} \widehat{\mathfrak{d}})=0 .
$$

Therefore

$$
\frac{1}{2} \frac{\mathrm{d}}{\mathrm{d} t}\left(|v \rho \widehat{\mathfrak{a}}|^{2}-2 \Re(v \rho \widehat{a} \widehat{\mathfrak{d}})\right)+v \rho^{2}|\widehat{\mathfrak{a}}|^{2}-v \rho^{2}|\widehat{\mathfrak{d}}|^{2}+\widetilde{\alpha} \rho^{2} v \Re(\widehat{\mathfrak{t}} \widehat{\mathfrak{a}})=0 .
$$

Combining the above identities, we conclude that for any $K \in \mathbb{R}$, we have

$$
\frac{1}{2} \frac{\mathrm{d}}{\mathrm{d} t} \mathcal{L}_{\rho}^{2}+v \rho^{2}|\widehat{\mathfrak{a}}|^{2}+(K \alpha-1) v \rho^{2}|\widehat{\mathfrak{d}}|^{2}+K \widetilde{\alpha} \kappa \rho^{2}|\widehat{\mathfrak{t}}|^{2}+\widetilde{\alpha} v \rho^{2} \Re(\widehat{\mathfrak{t}} \widehat{\mathfrak{a}})=0
$$

with

$$
\mathcal{L}_{\rho}^{2}:=K \alpha|(\widehat{\mathfrak{a}}, \widehat{\mathfrak{d}})|^{2}+K \widetilde{\alpha}|\widehat{\mathfrak{t}}|^{2}+|v \rho \widehat{\mathfrak{a}}|^{2}-2 \mathfrak{R}(v \rho \widehat{\mathfrak{a}} \widehat{\mathfrak{d}})
$$

Because

$$
\left|\widetilde{\alpha} v \rho^{2} \Re(\widehat{\mathfrak{t}} \widehat{\mathfrak{a}})\right| \leq \frac{v \rho^{2}}{2}|\widehat{\mathfrak{a}}|^{2}+\frac{\widetilde{\alpha}^{2} v \rho^{2}}{2}|\widehat{\mathfrak{t}}|^{2},
$$

we see that if we choose

$$
K \geq \max \left(\frac{2}{\alpha}, \frac{v \widetilde{\alpha}}{\kappa}\right),
$$

then we have $\mathcal{L}_{\rho} \approx|v \rho \widehat{\mathfrak{a}}|+\max (1, \sqrt{\nu / \kappa})|(\widehat{\mathfrak{a}}, \widehat{\mathfrak{d}}, \widehat{\mathfrak{t}})|$ and

$$
v \rho^{2}|\widehat{\mathfrak{a}}|^{2}+\left.(K \alpha-1) v \rho^{2} \widehat{\mathfrak{d}}\right|^{2}+K \widetilde{\alpha} \kappa \rho^{2}|\widehat{\mathfrak{t}}|^{2}+\widetilde{\alpha} v \rho^{2} \Re(\widehat{\mathfrak{t}} \widehat{\mathfrak{a}}) \gtrsim v \rho^{2}|(\widehat{\mathfrak{a}}, \widehat{\mathfrak{d}}, \widehat{\mathfrak{t}})|^{2} .
$$

Therefore if $v \lesssim \kappa$ then we get

$$
\mathcal{L}_{\rho}(t) \lesssim \mathcal{L}_{\rho}(0) e^{-c \min \left(\frac{1}{\nu}, v \rho^{2}\right) t},
$$

whence plugging this information in the equations for $\widehat{t}$ and for $\widehat{\mathfrak{d}}$ and using Duhamel's formula (to handle nonzero $\widehat{k}$ ), we end up with

$$
\left.|(\widehat{\mathfrak{a}}, \widehat{\mathfrak{d}}, \widehat{\mathfrak{t}})(t)|+v \rho^{2} \int_{0}^{t}|(\widehat{\mathfrak{a}}, \widehat{\mathfrak{d}}, \widehat{\mathfrak{t}})| \mathrm{d} \tau \leq K(\mid \widehat{\mathfrak{a}}, \widehat{\mathfrak{d}}, \widehat{\mathfrak{t}})(0)\left|+\int_{0}^{t}\right| \widehat{k} \mid \mathrm{d} \tau\right) \quad \text { if } \quad v \rho \leq 1,
$$

and

$$
\begin{aligned}
& |(v \rho \widehat{\mathfrak{a}}, \widehat{\mathfrak{d}}, \widehat{\mathfrak{t}})(t)|+\int_{0}^{t}|\rho \widehat{\mathfrak{a}}| \mathrm{d} \tau+v \rho^{2} \int_{0}^{t}|\widehat{\mathfrak{d}}| \mathrm{d} \tau+\kappa \rho^{2} \int_{0}^{t} \widehat{\mathfrak{t} \mid} \mathrm{d} \tau \\
& \leq K\left(|(v \rho \widehat{\mathfrak{a}}, \widehat{\mathfrak{d}}, \widehat{\mathfrak{t}})(0)|+\int_{0}^{t}|\rho v \widehat{k}| \mathrm{d} \tau\right) \text { if } v \rho \geq 1 .
\end{aligned}
$$

In the case $\kappa \lesssim v$, similar computations lead to

$$
|(\widehat{\mathfrak{a}}, \widehat{\mathfrak{d}}, \widehat{\mathfrak{t}})(t)|+\kappa \rho^{2} \int_{0}^{t}|(\widehat{\mathfrak{a}}, \widehat{\mathfrak{d}}, \widehat{\mathfrak{t}})| \mathrm{d} \tau \leq K\left(|(\widehat{\mathfrak{a}}, \widehat{\mathfrak{d}}, \widehat{\mathfrak{t}})(0)|+\int_{0}^{t}|\widehat{k}| \mathrm{d} \tau\right) \quad \text { if } \quad \sqrt{\kappa \nu} \rho \leq 1,
$$


and to

$$
\begin{aligned}
& |(\sqrt{\kappa v} \rho \widehat{\mathfrak{a}}, \widehat{\mathfrak{d}}, \widehat{\mathfrak{t}})(t)|+\sqrt{\frac{\kappa}{v}} \int_{0}^{t}|\rho \widehat{\mathfrak{a}}| \mathrm{d} \tau+\kappa \rho^{2} \int_{0}^{t}|\widehat{\mathfrak{d}}| \mathrm{d} \tau+\sqrt{\frac{\kappa}{v}} \kappa \rho^{2} \int_{0}^{t}|\widehat{\mathfrak{t}}| \mathrm{d} \tau \\
& \leq K\left(|(\sqrt{\kappa v} \rho \widehat{\mathfrak{a}}, \widehat{\mathfrak{d}}, \widehat{\mathfrak{t}})(0)|+\int_{0}^{t}|\sqrt{\kappa \nu} \rho \widehat{k}| \mathrm{d} \tau\right) \quad \text { if } \quad \kappa \rho \geq 1 .
\end{aligned}
$$

Remembering that $\widehat{k}=\varepsilon \rho^{2} \widehat{\mathfrak{t}}$, we easily conclude from the above inequalities that the solution to (50) fulfills

$\left.|(\rho \nu \widehat{\mathfrak{a}}, \widehat{\mathfrak{a}}, \widehat{\mathfrak{d}}, \widehat{\mathfrak{t}})(t)|+\min (1, v \rho) \int_{0}^{t}|\rho \widehat{\mathfrak{a}}| \mathrm{d} \tau+v \rho^{2} \int_{0}^{t} \mid \widehat{\mathfrak{d}}, \widehat{\mathfrak{t}}\right)|\mathrm{d} \tau \leq K|(\rho \nu \widehat{\mathfrak{a}}, \widehat{\mathfrak{a}}, \widehat{\mathfrak{d}}, \widehat{\mathfrak{t}})(0) \mid$ if $\nu \leq \kappa$

and

$$
\begin{aligned}
& |(\sqrt{\kappa v} \rho \widehat{\mathfrak{a}}, \widehat{\mathfrak{a}}, \widehat{\mathfrak{d}}, \widehat{\mathfrak{t}})(t)|+\min (1, \sqrt{\kappa v} \rho) \sqrt{\frac{\kappa}{v}} \int_{0}^{t}|\rho \widehat{\mathfrak{a}}| \mathrm{d} \tau \\
& \quad+\kappa \rho^{2} \int_{0}^{t}|\widehat{\mathfrak{t}}| \mathrm{d} \tau+\sqrt{\frac{\kappa}{v}} \kappa \rho^{2} \int_{0}^{t}|\widehat{\mathfrak{t}}| \mathrm{d} \tau \leq K|(\sqrt{\kappa v} \rho \widehat{\mathfrak{a}}, \widehat{\mathfrak{a}}, \widehat{\mathfrak{d}}, \widehat{\mathfrak{t}})(0)| \quad \text { if } \kappa \leq v
\end{aligned}
$$

provided that $\varepsilon \ll \min (\kappa, v)$ and $\rho \ll \varepsilon^{-1 / 2}$.

Let us finally resume to the proof of global-in-time decay estimates for the solution to (40). For notational simplicity, we do not track the dependency with respect to $\kappa$ and to $v$ any longer. We focus on the case where coefficients $\eta_{3}$ to $\eta_{8}$ are of order $\mathcal{C}$ and $\bar{\kappa}, \bar{v}$ and $\eta_{2}$ are of order 1 . This implies that $\varepsilon$ is of order $\mathcal{C}^{-1}$, and we thus get for all $\rho \ll \mathcal{C}$,

$$
|(\widehat{f}, \widehat{g}, \widehat{h})| \leq K \rho^{2}\left|V_{2}\right|+K \mathcal{C}^{-1} \rho^{2}|V| \text { and }|(\widehat{k}, \widehat{\ell})| \leq K \rho^{2}\left|V_{1}\right|+K \mathcal{C}^{-1} \rho^{2}|V| .
$$

Hence Inequalities (60), (64) and (65) (combined with Duhamel formula) yield for $\rho \lesssim 1, c$ small enough and $\mathcal{C}$ large enough,

$$
\begin{aligned}
& \left|V_{1}(t)\right|+c \rho^{2} \int_{0}^{t}\left|V_{1}\right| \mathrm{d} \tau \leq K V_{1}(0)+K \rho^{2} \int_{0}^{t}\left|V_{2}\right| \mathrm{d} \tau \\
& \text { and }\left|V_{2}(t)\right|+c \mathcal{C} \int_{0}^{t}\left|V_{2}\right| \mathrm{d} \tau \leq K V_{2}(0)+K \rho^{2} \int_{0}^{t}\left|V_{1}\right| \mathrm{d} \tau
\end{aligned}
$$

whence

$$
|V(t)|+\rho^{2} \int_{0}^{t}\left|V_{1}\right| \mathrm{d} \tau+\mathcal{C} \int_{0}^{t}\left|V_{2}\right| \mathrm{d} \tau \leq K|V(0)| .
$$

According to (64) and (65), for $\rho \gtrsim 1$, working with $\widetilde{V}_{1}:=(\rho \widehat{\mathfrak{a}}, \widehat{\mathfrak{d}}, \widehat{\mathfrak{t}})$ instead of $V_{1}=(\widehat{\mathfrak{a}}, \widehat{\mathfrak{d}}, \widehat{\mathfrak{t}})$ is more appropriate, and we thus have to use the following inequality (that stems from (65) and Duhamel formula):

$$
\left.\left|\widetilde{V}_{1}(t)\right|+\int_{0}^{t}|\rho \widehat{\mathfrak{a}}| \mathrm{d} \tau+\rho^{2} \int_{0}^{t} \mid \widehat{\mathfrak{d}}, \widehat{\mathfrak{t}}\right) \mid \mathrm{d} \tau \leq K\left(\left|\widetilde{V}_{1}(0)+\int_{0}^{t}\right|(\rho \widehat{f}, \widehat{g}, \widehat{h}) \mid \mathrm{d} \tau\right) .
$$

A closer look at the structure of $B_{2}^{12}$ and of $(\widehat{f}, \widehat{g}, \widehat{h})$ defined to be the r.h.s. of (47) reveals that

$$
|\rho \widehat{f}| \lesssim \mathcal{C}^{-1} \rho^{3}\left|\widehat{\mathrm{j}}_{0}\right|+\mathcal{C}^{-1} \rho^{4}|V| \text { and }|(\widehat{g}, \widehat{h})| \lesssim \rho^{2}\left|V_{2}\right|+\mathcal{C}^{-1} \rho^{3}|V|
$$


Hence inequality (66) implies that for $1 \lesssim \rho \ll \mathcal{C}^{1 / 3}$, we have

$$
\left.\left|\widetilde{V}_{1}(t)\right|+\int_{0}^{t}|\rho \widehat{\mathfrak{a}}| \mathrm{d} \tau+\rho^{2} \int_{0}^{t} \mid \widehat{\mathfrak{d}}, \widehat{\mathfrak{t}}\right) \mid \mathrm{d} \tau \leq K\left(\left|\widetilde{V}_{1}(0)\right|+\rho^{2} \int_{0}^{t}\left|V_{2}\right| \mathrm{d} \tau\right) .
$$

It is also clear that for $1 \lesssim \rho \lesssim \mathcal{C}^{-1}$,

$$
\left.\left.|(\widehat{k}, \widehat{\ell})| \lesssim \mathcal{C}^{-1} \rho^{2}|\widehat{\mathfrak{a}}|+\rho^{2} \mid \widehat{\mathfrak{d}}, \widehat{\mathfrak{t}}\right)\left|+\mathcal{C}^{-1} \rho^{3}\right| V\left|\lesssim \mathcal{C}^{-1} \rho^{2}\right| \rho \widehat{\mathfrak{a}}\left|+\rho^{2}\right| \widehat{\mathfrak{d}}, \widehat{\mathfrak{t}}\right)\left|+\mathcal{C}^{-1} \rho^{3}\right| V_{2} \mid
$$

Hence if $\rho \ll \mathcal{C}^{2 / 3}$,

$$
\left.\left|V_{2}(t)\right|+\mathcal{C} \int_{0}^{t}\left|V_{2}\right| \mathrm{d} \tau \leq K\left(\left|V_{2}(0)\right|+\mathcal{C}^{-1} \rho^{2} \int_{0}^{t}|\rho \widehat{a}| \mathrm{d} \tau+\rho^{2} \int_{0}^{t} \mid \widehat{\mathfrak{d}}, \widehat{\mathfrak{t}}\right) \mid \mathrm{d} \tau\right) .
$$

Inserting (67) in (68), it is now easy to conclude that for $1 \lesssim \rho \ll \mathcal{C}^{1 / 3}$, we have

$$
\left|V_{2}(t)\right|+\mathcal{C} \int_{0}^{t}\left|V_{2}\right| \mathrm{d} \tau \leq K\left|\left(\widetilde{V}_{1}, V_{2}\right)(0)\right|,
$$

and thus

$$
\left.\left|\widetilde{V}_{1}(t)\right|+\int_{0}^{t}|\rho \widehat{\mathfrak{a}}| \mathrm{d} \tau+\rho^{2} \int_{0}^{t} \mid \widehat{\mathfrak{d}}, \widehat{\mathfrak{t}}\right)|\mathrm{d} \tau \leq K|\left(\widetilde{V}_{1}, V_{2}\right)(0) \mid .
$$

The case where there is some source term $\widehat{F}=\left(\widehat{A}, \widehat{D}, \widehat{\Theta}, \widehat{J_{0}}, \widehat{J_{1}}\right)$ in (40) may be treated along the same lines, and we end up for all $0 \leq \rho \ll \mathcal{C}^{1 / 3}$ and $t \geq 0$ with

$$
\begin{aligned}
& \left.\left.\left|\left(\widehat{\mathfrak{a}}, \rho \widehat{\mathfrak{a}}, \widehat{\mathfrak{d}}, \widehat{\mathfrak{t}}, \widehat{\mathfrak{j}_{0}}, \widehat{j_{1}}\right)(t)\right|+\min (1, \rho) \int_{0}^{t}|\rho \widehat{\mathfrak{a}}| \mathrm{d} \tau+\rho^{2} \int_{0}^{t} \mid \widehat{\mathfrak{d}}, \widehat{\mathfrak{t}}\right)\left|\mathrm{d} \tau+\mathcal{C} \int_{0}^{t}\right| \widehat{\mathfrak{j}_{0}}, \widehat{j_{1}}\right) \mid \mathrm{d} \tau \\
& \quad \leq K\left(\left|\left(\widehat{\mathfrak{a}}, \rho \widehat{\mathfrak{a}}, \widehat{\mathfrak{d}}, \widehat{\mathfrak{t}}, \widehat{j_{0}}, \widehat{\mathfrak{j}_{1}}\right)(0)\right|+\int_{0}^{t}\left|\left(\widehat{A}, \rho \widehat{A}, \widehat{D}, \widehat{\Theta}, \widehat{J_{0}}, \widehat{J_{1}}\right)\right| \mathrm{d} \tau\right) .
\end{aligned}
$$

\subsection{Middle-frequency decay estimates}

This paragraph is devoted to the proof of global-in-time estimates for the solution to (40) in some suitable frequency range $\rho_{\ell} \leq \rho \leq \rho_{h}$ where $\rho_{h}$ is prescribed and $\rho_{\ell}$ will be specified below. Having in mind the study of the nonlinear system (25), it is natural to work at the same level regularity for $|D| a, d,|D|^{-1} \Theta, j_{0}$ and $j_{1}$ which, in Fourier variables, corresponds to $\left(\rho \widehat{a}, \widehat{d}, \rho^{-1} \widehat{\Theta}, \widehat{j_{0}}, \widehat{j_{1}}\right)$. We thus introduce $\widehat{\phi}:=\rho^{-1} \widehat{\Theta}$. In terms of $\left(\widehat{a}, \widehat{d}, \widehat{\phi}, \widehat{j_{0}}, \widehat{j_{1}}\right)$, System (40) rewrites

$$
\frac{\mathrm{d}}{\mathrm{d} t}\left(\begin{array}{c}
\widehat{a} \\
\widehat{d} \\
\widehat{\phi} \\
\widehat{j_{0}} \\
\widehat{j_{1}}
\end{array}\right)+\left(\begin{array}{ccccc}
0 & \rho & 0 & 0 & 0 \\
-\rho & \bar{\nu} \rho^{2} & -\rho^{2} & 0 & -1 \\
0 & \eta_{2} & \eta_{3}+\bar{\kappa} \rho^{2} & -\eta_{4} \rho^{-1} & 0 \\
0 & 0 & -\eta_{7} \rho & \eta_{5} & \eta_{6} \rho \\
0 & 0 & 0 & -\eta_{6} \rho & \eta_{8}
\end{array}\right)\left(\begin{array}{c}
\widehat{a} \\
\widehat{d} \\
\widehat{\phi} \\
\widehat{j_{0}} \\
\widehat{j_{1}}
\end{array}\right)=\left(\begin{array}{l}
0 \\
0 \\
0 \\
0 \\
0
\end{array}\right) .
$$

To handle $(\widehat{a}, \widehat{d})$, it is only a matter of using the standard barotropic estimates, considering the coupling with $\widehat{\phi}$ and $\widehat{j_{1}}$ as source terms. More precisely, taking advantage of the Lyapunov functional

$$
2\left(|\widehat{a}|^{2}+|\widehat{d}|^{2}\right)+|v \rho \widehat{a}|^{2}-2 v \rho \Re(\widehat{d} \widehat{a})
$$


we get for $\rho v \geq \rho_{\ell}$ (for any given $\rho_{\ell}>0$ ),

$|(\bar{v} \rho \widehat{a}, \widehat{d})(t)|+\int_{0}^{t}|\rho \widehat{a}| \mathrm{d} \tau+\bar{v} \rho^{2} \int_{0}^{t}|\widehat{d}| \mathrm{d} \tau \leq C\left(|(\bar{v} \rho \widehat{a}, \widehat{d})(0)|+\int_{0}^{t}\left|\widehat{j_{1}}\right| \mathrm{d} \tau+\int_{0}^{t}\left|\rho^{2} \widehat{\phi}\right| \mathrm{d} \tau\right)$

for some constant $C$ depending only on $\rho_{\ell}$.

Let us now concentrate on the system fulfilled by $\left(\widehat{\phi}, \widehat{j_{0}}, \widehat{j_{1}}\right)$, namely,

$$
\left\{\begin{array}{l}
\partial_{t} \widehat{\phi}+\left(\eta_{3}+\bar{\kappa} \rho^{2}\right) \widehat{\phi}-\eta_{4} \rho^{-1} \widehat{j_{0}}=\widehat{\Phi}:=-\eta_{2} \widehat{d}, \\
\partial_{t} \widehat{j_{0}}+\eta_{5} \widehat{j_{0}}+\eta_{6} \rho \widehat{j_{1}}-\eta_{7} \rho \widehat{\phi}=0, \\
\partial_{t} \hat{j_{1}}+\eta_{8} \widehat{j_{1}}-\eta_{6} \rho \widehat{j_{0}}=0 .
\end{array}\right.
$$

Let us put $\widehat{\Phi}$ to 0 for a while. In order to get decay estimates for $\left(\widehat{\phi}, \widehat{j_{0}}, \widehat{j_{1}}\right)$, we multiply the equation for $\widehat{\phi}$ by $\widehat{\phi}$, and get:

$$
\frac{1}{2} \frac{\mathrm{d}}{\mathrm{d} t}|\widehat{\phi}|^{2}+\left(\eta_{3}+\bar{\kappa} \rho^{2}\right)|\widehat{\phi}|^{2}-\eta_{4} \rho^{-1} \Re\left(\widehat{j_{0}} \widehat{\widehat{\phi}}\right)=0 .
$$

Next, taking the inner product in $\mathbb{C}^{2}$ of the equations for $\left(\widehat{j_{0}}, \widehat{j_{1}}\right)$ with $\left(\widehat{j_{0}}, \widehat{j_{1}}\right)$, we find out that

$$
\frac{1}{2} \frac{\mathrm{d}}{\mathrm{d} t}\left|\left(\widehat{j_{0}}, \widehat{j_{1}}\right)\right|^{2}+\eta_{5}\left|\widehat{j_{0}}\right|^{2}+\eta_{8}\left|\widehat{j_{1}}\right|^{2}-\eta_{7} \rho \Re\left(\widehat{j_{0}} \widehat{\bar{\phi}}\right)=0 .
$$

In order to eliminate the last term (which tends to be predominant for large $\rho$ ), we compute

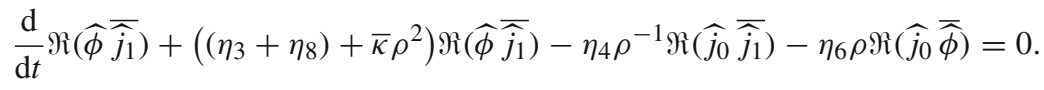

Hence setting for some $A>0$ to be fixed hereafter,

$$
\mathcal{L}_{\rho}^{2}:=A|\widehat{\phi}|^{2}+\left|\left(\widehat{j_{0}}, \widehat{j_{1}}\right)\right|^{2}-2 B \Re\left(\widehat{\phi} \widehat{\dot{j}_{1}}\right) \text { with } B:=\frac{1}{\eta_{6}}\left(\eta_{7}+A \frac{\eta_{4}}{\rho^{2}}\right),
$$

we discover that

$$
\begin{aligned}
& \frac{1}{2} \frac{\mathrm{d}}{\mathrm{d} t} \mathcal{L}_{\rho}^{2}+A\left(\eta_{3}+\bar{\kappa} \rho^{2}\right)|\widehat{\phi}|^{2}+\eta_{5}\left|\widehat{j_{0}}\right|^{2}+\eta_{8}\left|\widehat{j_{1}}\right|^{2} \\
& \quad-B\left(\eta_{3}+\eta_{8}+\bar{\kappa} \rho^{2}\right) \Re\left(\widehat{\phi} \widehat{\widehat{j_{1}}}\right)+B \eta_{4} \rho^{-1} \Re\left(\widehat{j_{0}} \widehat{\widehat{j_{1}}}\right)=0 .
\end{aligned}
$$

Note that we have

$$
B \leq \frac{2 \eta_{7}}{\eta_{4}} \text { if } \rho^{2} \geq \frac{\eta_{4}}{\eta_{7}} A,
$$

and thus, as may be easily seen by Young inequality,

$$
\mathcal{L}_{\rho}^{2} \approx\left|\left(\widehat{j_{0}}, \widehat{j_{1}}, \widehat{\phi}\right)\right|^{2} \text { for } \rho \geq \sqrt{\frac{\eta_{4}}{\eta_{7}} A}
$$

if $A$ has been chosen so that

$$
A>8\left(\frac{\eta_{7}}{\eta_{6}}\right)^{2}
$$

Next, we see that by virtue of (76), we have

$$
B \eta_{4} \rho^{-1}\left|\Re\left(\widehat{j_{0}} \widehat{\hat{j}_{1}}\right)\right| \leq 2 \frac{\eta_{4} \eta_{7}}{\eta_{6} \rho}\left|\widehat{j_{0}}\right|\left|\widehat{j_{1}}\right|,
$$


whence

$$
B \eta_{4} \rho^{-1}\left|\Re\left(\widehat{j_{0}} \widehat{\dot{j}_{1}}\right)\right| \leq \frac{\min \left(\eta_{5}, \eta_{8}\right)}{4}\left|\left(\widehat{j_{0}}, \widehat{j_{1}}\right)\right|^{2} \text { if in addition } \rho \geq \frac{4 \eta_{4} \eta_{7}}{\eta_{6} \min \left(\eta_{5}, \eta_{8}\right)} .
$$

Finally, still using (76), we have

$$
B\left(\eta_{3}+\eta_{8}+\bar{\kappa} \rho^{2}\right)\left|\Re\left(\widehat{\phi} \widehat{j_{1}}\right)\right| \leq \frac{\min \left(\eta_{5}, \eta_{8}\right)}{2}\left|\widehat{j_{1}}\right|^{2}+\frac{2}{\min \left(\eta_{5}, \eta_{8}\right)}\left(\frac{\eta_{7}\left(\eta_{3}+\eta_{8}+\bar{\kappa} \rho^{2}\right)}{\eta_{6}}\right)^{2}|\widehat{\phi}|^{2} .
$$

As we want the last term to be bounded by $\frac{A}{2}\left(\eta_{3}+\bar{\kappa} \rho^{2}\right)|\widehat{\phi}|^{2}$, we eventually require $A$ to be chosen so that

$$
\frac{2}{\min \left(\eta_{5}, \eta_{8}\right)}\left(\frac{\eta_{7}\left(\eta_{3}+\eta_{8}+\bar{\kappa} \rho^{2}\right)}{\eta_{6}}\right)^{2} \leq \frac{A}{2}\left(\eta_{3}+\bar{\kappa} \rho^{2}\right) \text { for all } \rho \leq \rho_{h} .
$$

Easy computations show that a sufficient condition for that is

$$
A \geq 4\left(\frac{\left(\eta_{3}+\eta_{8}\right) \eta_{7}}{\eta_{3} \eta_{6}}\right)^{2}\left(\frac{\eta_{3}+\bar{\kappa} \rho_{h}^{2}}{\min \left(\eta_{5}, \eta_{8}\right)}\right) .
$$

Let us sum up: our computations show that if $A$ has been chosen so that (77) and (79) are fulfilled, and if we assume that

$$
\min \left(\sqrt{\frac{\eta_{4}}{\eta_{7}} A}, \frac{4 \eta_{4} \eta_{7}}{\eta_{6} \min \left(\eta_{5}, \eta_{8}\right)}\right) \leq \rho \leq \rho_{h}
$$

where $\rho_{h}$ is given, then we have $\mathcal{L}_{\rho} \approx \mid\left(\widehat{j_{0}}, \widehat{j_{1}}, \widehat{\phi}\right)$ and

$$
\frac{\mathrm{d}}{\mathrm{d} t} \mathcal{L}_{\rho}^{2}+A\left(\eta_{3}+\bar{\kappa} \rho^{2}\right)|\widehat{\phi}|^{2}+\min \left(\eta_{5}, \eta_{8}\right)\left|\left(\widehat{j_{0}}, \widehat{j_{1}}\right)\right|^{2} \leq 0 .
$$

Let us recall that all the coefficients $\eta_{i}$ with $3 \leq i \leq 8$ are of the same order $\mathcal{C}$. Therefore one can take $A$ of order 1 whenever $\bar{\kappa} \rho_{h}^{2}$ is of order $\mathcal{C}$, and thus for all $\rho$ satisfying (80) and $t \geq 0$,

$$
\left|\left(\widehat{\phi}, \widehat{j_{0}}, \widehat{j_{1}}\right)(t)\right| \leq C_{0} e^{-c_{0} \mathcal{C} t}\left|\left(\widehat{\phi}, \widehat{j_{0}}, \widehat{j_{1}}\right)(0)\right| .
$$

From that inequality, we conclude thanks to Duhamel's formula that if we take the r.h.s. $\Phi=-\eta_{2} \widehat{d}$ into account then we have

$$
\left|\left(\widehat{\phi}, \widehat{j_{0}}, \widehat{j_{1}}\right)(t)\right|+\mathcal{C} \int_{0}^{t}\left|\left(\widehat{\phi}, \widehat{j_{0}}, \widehat{j_{1}}\right)\right| \mathrm{d} \tau \leq C_{0}\left(\left|\left(\widehat{\phi}, \widehat{j_{0}}, \widehat{j_{1}}\right)(0)\right|+\rho \int_{0}^{t}|\widehat{d}| \mathrm{d} \tau\right) .
$$

Inserting that inequality in (71), we conclude that for all $\rho_{0}>0$ there exists a constant $K$ depending only on $\eta_{2}$ and on the renormalized coefficients $\eta_{i}^{\prime}:=\eta_{i} / \mathcal{C}$ for $2 \leq i \leq 8$ so that if

$$
\min \left(\frac{1}{\bar{\nu}}, \frac{1}{\sqrt{\overline{\kappa \nu}}}\right) \leq \rho \leq \rho_{0} \sqrt{\frac{\mathcal{C}}{\bar{\kappa}}},
$$

then we have for all $t \geq 0$ and large enough $\mathcal{C}$,

$$
\begin{aligned}
& \bar{\kappa}|(\bar{v} \rho \widehat{a}, \widehat{d})(t)|+\left|\left(\rho^{-1} \widehat{\Theta}, \widehat{j_{0}}, \widehat{j_{1}}\right)(t)\right|+\kappa \rho \int_{0}^{t}|\widehat{a}| \mathrm{d} \tau+\bar{v} \rho^{2} \int_{0}^{t}|\widehat{d}| \mathrm{d} \tau \\
& \quad+\mathcal{C} \int_{0}^{t}\left|\left(\rho^{-1} \Theta, \widehat{j_{0}}, \widehat{j_{1}}\right)\right| \mathrm{d} \tau \leq K\left(\bar{\kappa}|(\bar{v} \rho \widehat{a}, \widehat{d})(0)|+\left|\left(\rho^{-1} \widehat{\Theta}, \widehat{j_{0}}, \widehat{j_{1}}\right)(0)\right|\right) .
\end{aligned}
$$




\subsection{High-frequency decay estimates}

We now want to prove global-in-time estimates for the solution to (40) in the case $\rho \gtrsim \sqrt{\mathcal{C} / \bar{\kappa}}$. To this end, as for middle frequencies, it is convenient to work with $\widehat{\phi}=\rho^{-1} \widehat{\Theta}$ rather than with $\widehat{\Theta}$. Now from the equation satisfied by $\widehat{\phi}$ in (70), we readily get

$$
|\widehat{\phi}(t)|+\bar{\kappa} \rho^{2} \int_{0}^{t}|\widehat{\phi}| \mathrm{d} \tau \leq\left|\widehat{\phi}_{0}\right|+\eta_{2} \int_{0}^{t}|\widehat{d}| \mathrm{d} \tau+\eta_{4} \rho^{-1} \int_{0}^{t}\left|\widehat{j_{0}}\right| \mathrm{d} \tau
$$

while the last two lines of (70) yield

$$
\left|\left(\widehat{j_{0}}, \widehat{j_{1}}\right)(t)\right|+\min \left(\eta_{5}, \eta_{8}\right) \int_{0}^{t}\left|\left(\widehat{j_{0}}, \widehat{j_{1}}\right)\right| \mathrm{d} \tau \leq\left|\left(\widehat{j_{0}}, \widehat{j_{1}}\right)(0)\right|+\eta_{7} \rho \int_{0}^{t}|\widehat{\phi}| \mathrm{d} \tau .
$$

Inserting (84) in (85) and omitting from now the dependency with respect to the coefficients $\eta_{2}$ and $\eta_{i}^{\prime}$, we discover that for $\rho^{2} \gg \bar{\kappa}^{-1} \mathcal{C}$,

$$
\left|\left(\widehat{j_{0}}, \widehat{j_{1}}\right)(t)\right|+\mathcal{C} \int_{0}^{t}\left|\left(\widehat{j_{0}}, \widehat{j_{1}}\right)\right| \mathrm{d} \tau \lesssim\left|\left(\widehat{j_{0}}, \widehat{j_{1}}\right)(0)\right|+\frac{\mathcal{C}}{\bar{\kappa} \rho}\left(\left|\widehat{\phi}_{0}\right|+\int_{0}^{t}|\widehat{d}| \mathrm{d} \tau\right),
$$

and thus, resuming to (84),

$$
|\widehat{\phi}(t)|+\bar{\kappa} \rho^{2} \int_{0}^{t}|\widehat{\phi}| \mathrm{d} \tau \lesssim\left|\widehat{\phi}_{0}\right|+\rho^{-1}\left|\left(\widehat{j_{0}}, \widehat{j_{1}}\right)(0)\right|+\int_{0}^{t}|\widehat{d}| \mathrm{d} \tau .
$$

In order to bound $(\widehat{a}, \widehat{d})$, we combine (71), (86) and (87), so as to get

$$
\begin{aligned}
& |(\bar{v} \rho \widehat{a}, \widehat{d})(t)|+\int_{0}^{t}|\rho \widehat{a}| \mathrm{d} \tau+\bar{v} \rho^{2} \int_{0}^{t}|\widehat{d}| \mathrm{d} \tau \lesssim|(\bar{v} \rho \widehat{a}, \widehat{d})(0)|+\bar{\kappa}^{-1} \rho^{-1}\left|\left(\rho \widehat{\phi}, \widehat{j_{0}}, \widehat{j_{1}}\right)(0)\right| \\
& \quad+\mathcal{C}^{-1}\left|\left(\widehat{j_{0}}, \widehat{j_{1}}\right)(0)\right|+\left(\bar{\kappa} \rho^{2}\right)^{-1}\left|\rho \widehat{\phi}_{0}\right|+\bar{\kappa}^{-1}\left(1+\rho^{-1}\right) \int_{0}^{t}|\widehat{d}| \mathrm{d} \tau .
\end{aligned}
$$

The last term may be absorbed by the 1.h.s. provided that $1+\rho^{-1} \ll \overline{\kappa \nu} \rho^{2}$. Resuming to (86) and (87), and remembering that $\widehat{\Theta}=\rho \widehat{\phi}$, we conclude that

$$
\begin{aligned}
& \left|\left(\bar{v} \rho \widehat{a}, \widehat{d},(\bar{\kappa} \rho)^{-1} \widehat{\Theta}, \widehat{j_{0}}, \widehat{j_{1}}\right)(t)\right|+\rho \int_{0}^{t}|(\widehat{a}, \widehat{\Theta})| \mathrm{d} \tau+\bar{v} \rho^{2} \int_{0}^{t}|\widehat{d}| \mathrm{d} \tau+\mathcal{C} \int_{0}^{t}\left|\left(\widehat{j_{0}}, \widehat{j_{1}}\right)\right| \mathrm{d} \tau \\
& \quad \lesssim\left(\left|\left(\bar{v} \rho \widehat{a}, \widehat{d},(\bar{\kappa} \rho)^{-1} \widehat{\Theta}, \widehat{j_{0}}, \widehat{j_{1}}\right)(0)\right| \quad \text { for } \rho \gg \sqrt{\mathcal{C} / \bar{\kappa}} \text { and } \mathcal{C} \gg 1 .\right.
\end{aligned}
$$

\subsection{Final statement of linear estimates}

Here we recap the estimates that we obtained so far for (40), if $\mathcal{C} \gg 1$.

To this end, we first fix $\mathcal{C}_{0}$ and $\rho_{h}$ large enough so that (88) holds true for any $\mathcal{C} \geq \mathcal{C}_{0}$ and $\rho \geq \rho_{h} \sqrt{\mathcal{C} / \bar{\kappa}}$. Then the analysis for the middle frequency ensures that, taking $\mathcal{C}_{0}$ larger if needed, Inequality (83) holds true for $\mathcal{C} \geq \mathcal{C}_{0}$ and $\rho_{\ell} \leq \rho \leq 2 \rho_{h} \sqrt{\mathcal{C} / \bar{\kappa}}$ for some $\rho_{\ell}$ of order 1 depending only on the parameters of the system. Finally, for the low frequencies, one can use (69) for $\rho \leq 2 \rho_{\ell}$ if $\mathcal{C}$ is large enough.

We thus eventually have for $\mathcal{C} \geq \mathcal{C}_{0}$ the following three inequalities: $:^{5}$

- Low frequencies $0 \leq \rho \leq 2 \rho_{\ell}$ : From (69) and (45), we get

5 We refrain from tracking the dependency with respect to $\bar{\kappa}$ and $\bar{v}$. 


$$
\begin{aligned}
& \left|\left(\widehat{a}, \widehat{d}, \widehat{\Theta}, \widehat{j_{0}}, \widehat{j_{1}}\right)(t)\right|+\rho^{2} \int_{0}^{t}\left|\left(\widehat{a}, \widehat{d}, \widehat{\Theta}, \widehat{j_{0}}, \widehat{j_{1}}\right)\right| \mathrm{d} \tau \\
& \left.\quad+\mathcal{C} \int_{0}^{t} \mid \widehat{j_{0}}, \widehat{j_{1}}\right)|\mathrm{d} \tau \lesssim|\left(\widehat{a}, \widehat{d}, \widehat{\Theta}, \widehat{j_{0}}, \widehat{j_{1}}\right)(0) \mid
\end{aligned}
$$

with

$$
\widehat{j_{0}}:=\widehat{j_{0}}-\frac{\eta_{2} \eta_{3}}{\eta_{4}\left(\eta_{3}+\eta_{5}\right)} \rho\left(\widehat{d}+\frac{1}{\eta_{8}} \widehat{j_{1}}\right)-\frac{\eta_{3}}{\eta_{4}} \widehat{\Theta} .
$$

- Middle frequencies $\rho_{\ell} \leq \rho \leq 2 \rho_{h} \sqrt{\mathcal{C}}$ : Inequality (83) gives

$$
\begin{aligned}
& \left|\left(\rho \widehat{a}, \widehat{d}, \rho^{-1} \widehat{\Theta}, \widehat{j_{0}}, \widehat{j_{1}}\right)(t)\right|+\rho \int_{0}^{t}|\widehat{a}| \mathrm{d} \tau+\rho^{2} \int_{0}^{t}|\widehat{d}| \mathrm{d} \tau+\mathcal{C} \int_{0}^{t}\left|\left(\rho^{-1} \widehat{\Theta}, \widehat{j_{0}}, \widehat{j_{1}}\right)\right| \mathrm{d} \tau \\
& \quad \lesssim\left|\left(\rho \widehat{a}, \widehat{d}, \rho^{-1} \widehat{\Theta}, \widehat{j_{0}}, \widehat{j_{1}}\right)(0)\right| .
\end{aligned}
$$

- High frequencies $\rho \geq \rho_{h} \sqrt{\mathcal{C}}$ : Inequality (88) implies that

$$
\begin{aligned}
& \left|\left(\rho \widehat{a}, \widehat{d}, \rho^{-1} \widehat{\Theta}, \widehat{j_{0}}, \widehat{j_{1}}\right)(t)\right|+\rho \int_{0}^{t}|(\widehat{a}, \widehat{\Theta})| \mathrm{d} \tau+\rho^{2} \int_{0}^{t}|\widehat{d}| \mathrm{d} \tau+\mathcal{C} \int_{0}^{t}\left|\left(\widehat{j_{0}}, \widehat{j_{1}}\right)\right| \mathrm{d} \tau \\
& \quad \lesssim\left(\left|\left(\rho \widehat{a}, \widehat{d}, \rho^{-1} \widehat{\Theta}, \widehat{j_{0}}, \widehat{j_{1}}\right)(0)\right| .\right.
\end{aligned}
$$

Remark 3.1 Having a (small) overlap between the three regimes will be important in the sequel as the Fourier splitting device that we will use, namely the Littlewood-Paley decomposition, is not quite orthogonal.

Remark 3.2 Inequality (89) implies that for $0 \leq \rho \leq 2 \rho_{\ell}$, we have

$$
\mathcal{C} \int_{0}^{t}|\widehat{\zeta} 0| \mathrm{d} \tau \lesssim\left|\left(\widehat{a}, \widehat{d}, \widehat{\Theta}, \widehat{j_{0}}, \widehat{j_{1}}\right)(0)\right| \text { with } \widehat{\zeta}_{0}:=\widehat{j_{0}}-\frac{\eta_{2} \eta_{3}}{\eta_{4}\left(\eta_{3}+\eta_{5}\right)} \rho \widehat{d}-\frac{\eta_{3}}{\eta_{4}} \widehat{\Theta}
$$

Let us also emphasize that the inequality for middle frequencies is stronger than that for high frequencies as regards $\Theta$. Therefore (92) is fulfilled for all $\rho \geq \rho_{\ell}$.

Finally, regarding the incompressible part of the solution, namely $\left(\mathcal{P} \vec{u}, \mathcal{P} \vec{j}_{1}\right.$ ), we readily have from (33) that for all $\rho \geq 0$ and $\mathcal{C} \geq 0$,

$$
\begin{aligned}
& |\widehat{\mathcal{P} \vec{u}}(t)|+\rho^{2} \int_{0}^{t}|\widehat{\mathcal{P} \vec{u}}| \mathrm{d} \tau \leq K\left(|\widehat{\mathcal{P} \vec{u}}(0)|+\mathcal{C}^{-1}\left|\widehat{\mathcal{P}} \vec{j}_{1}(0)\right|\right) \\
& \text { and }\left|\widehat{\mathcal{P} \vec{j}_{1}}(t)\right|+\mathcal{C} \int_{0}^{t}\left|\widehat{\mathcal{P} \vec{j}_{1}}\right| \mathrm{d} \tau \leq K\left|\widehat{\mathcal{P} \vec{j}_{1}}(0)\right|
\end{aligned}
$$

\section{The paralinearized system}

In order to achieve the global existence result of Theorem 2.3, it is tempting to look at (25) as the linear system studied in the previous section. Indeed one expect to be able to handle the r.h.s. of (25) according to Duhamel's formula. This unfortunately does not work because some of the convection terms will cause a loss of one derivative in the estimates, exactly as for the compressible Euler or Navier-Stokes equations. Paralinearizing (25) (that is including the "principal" part of the convection terms) is a standard way to overcome this difficulty. In the case we are interested in, it turns out that only $\vec{u} \cdot \nabla a$ causes a loss of derivative because the other convection terms may be counterbalanced by the parobolicity of the equations of $\vec{u}$ 
and of $\Theta$. However, for symmetry reasons, it is convenient to paralinearize $\vec{u} \cdot \nabla \vec{u}$, too. This eventually leads to the following paralinearized version of System $^{6}(25)$ :

$$
\left\{\begin{array}{l}
\partial_{t} a+T_{\vec{v}} \cdot \nabla a+\operatorname{div} \vec{u}=F, \\
\partial_{t} \vec{u}+T_{\vec{v}} \cdot \nabla \vec{u}-\frac{1}{\operatorname{Re}} \underline{\mathcal{A}} \vec{u}+\frac{1}{M a^{2}} \underline{\alpha}_{1} \nabla a+\frac{1}{M a^{2}} \underline{\alpha}_{2} \nabla \Theta-\frac{1}{n} \mathcal{L}\left(\underline{\sigma}_{a}+\mathcal{L}_{s} \underline{\sigma}_{s}\right) \vec{j}_{1}=\vec{G}, \\
\partial_{t} \Theta-\frac{1}{\operatorname{Pr} R e} \underline{\kappa} \Delta \Theta+\underline{\alpha}_{2} \operatorname{div} \vec{u}-\frac{\mathcal{C} \mathcal{L}}{\operatorname{Pr}} \underline{\sigma}_{a}\left(j_{0}-\underline{\alpha}^{\prime} \Theta\right)=H, \\
\partial_{t} j_{0}+\frac{1}{n} \mathcal{C} \operatorname{div} \vec{j}_{1}+\mathcal{C} \mathcal{L} \underline{\sigma}_{a}\left(j_{0}-\underline{\alpha}^{\prime} \Theta\right)=J_{0}, \\
\partial_{t} \vec{j}_{1}+\mathcal{C} \nabla j_{0}+\mathcal{C} \mathcal{L}\left(\underline{\sigma}_{a}+\mathcal{L}_{s} \underline{\sigma}_{s}\right) \vec{j}_{1}=\vec{J}_{1},
\end{array}\right.
$$

where the velocity field $\vec{v}$ and the source terms $F, \vec{G}, H, J_{0}, \vec{J}_{1}$ are given. The reader may refer to the Appendix for the definition of $T_{\vec{v}} \cdot \nabla a$ and $T_{\vec{v}} \cdot \nabla \vec{u}$.

The main result of this part is stated in the following proposition:

Proposition 4.1 There exists a constant $\mathcal{C}_{0}>0$ and an integer $k_{0}$ depending only on the parameters of System (95) such that if

$$
\mathcal{C} \geq \mathcal{C}_{0}
$$

and the threshold between low and high frequencies is at $2^{k_{0}}$ (see (135)) then the following inequalities hold true for all $s$ and $s^{\prime}$ in $\mathbb{R}$ :

- Low frequencies:

$$
\begin{aligned}
& \left\|\left(a, \vec{u}, \Theta, j_{0}, \vec{j}_{1}\right)(t)\right\|_{\dot{B}_{2,1}^{s}}^{\ell}+\int_{0}^{t}\left(\left\|\left(a, \vec{u}, \Theta, j_{0}, \vec{j}_{1}\right)\right\|_{\dot{B}_{2,1}^{s+2}}^{\ell}+\mathcal{C}\left\|\left(\zeta_{0}, \vec{j}_{1}\right)\right\|_{\dot{B}_{2,1}^{s}}^{\ell}\right) \\
& \lesssim\left\|\left(a, \vec{u}, \Theta, j_{0}, \vec{j}_{1}\right)(0)\right\|_{\dot{B}_{2,1}^{s}}^{\ell}+\int_{0}^{t}\left\|\left(J_{0}, \vec{J}_{1}, H\right)\right\|_{\dot{B}_{2,1}^{s}}^{\ell} d \tau \\
& \quad+\int_{0}^{t}\left\|\left(F-T_{\vec{v}} \cdot \nabla a, \vec{G}-T_{\vec{v}} \cdot \nabla \vec{u}\right)\right\|_{\dot{B}_{2,1}^{s}}^{\ell} d \tau,
\end{aligned}
$$

with

$$
\zeta_{0}:=j_{0}-\underline{\alpha}^{\prime} \Theta-\frac{\underline{\alpha}_{2} \underline{\alpha}^{\prime}}{\underline{\alpha}_{1} \mathcal{C} \mathcal{L} \underline{\sigma}_{a}\left(1+\frac{1}{\operatorname{Pr}} \underline{\alpha}^{\prime}\right)} \operatorname{div} \vec{u} .
$$

- High frequencies:

$$
\begin{aligned}
& \left\|\left(\vec{u}, j_{0}, \vec{j}_{1}\right)(t)\right\|_{\dot{B}_{2,1}^{s^{\prime}}}^{h}+\|a(t)\|_{\dot{B}_{2,1}^{s^{\prime}+1}}^{h}+\|\Theta(t)\|_{\dot{B}_{2,1}^{s^{\prime}-1}}^{h}+\int_{0}^{t}\left(\|(a, \Theta)\|_{\dot{B}_{2,1}^{s^{\prime}+1}}^{h}+\|\vec{u}\|_{\dot{B}_{2,1}^{s^{\prime}+2}}^{h}\right) d \tau \\
& +\mathcal{C} \int_{0}^{t}\left(\|\Theta\|_{\dot{B}_{2,1}^{s^{\prime}-1}}^{h}+\left\|\left(j_{0}, \vec{j}_{1}\right)\right\|_{\dot{B}_{2,1}^{s^{\prime}}}^{h}\right) d \tau \lesssim\left\|\left(\vec{u}, j_{0}, \vec{j}_{1}\right)(0)\right\|_{\dot{B}_{2,1}^{s^{\prime}}}^{h} \\
& +\|a(0)\|_{\dot{B}_{2,1}^{s^{\prime}+1}}^{h}+\|\Theta(0)\|_{\dot{B}_{2,1}^{s^{\prime}-1}}^{h} \\
& +\int_{0}^{t}\left(\left\|\left(\vec{G}, J_{0}, \vec{J}_{1}\right)\right\|_{\dot{B}_{2,1}^{s^{\prime}}}^{h}+\|F\|_{\dot{B}_{2,1}^{s^{\prime}+1}}^{h}+\|H\|_{\dot{B}_{2,1}^{s^{\prime}-1}}^{h}\right) d \tau \\
& \quad+\int_{0}^{t} \nabla \vec{v} \|_{L^{\infty}}\left(\|(\nabla a, \vec{u})\|_{\dot{B}_{2,1}^{s^{\prime}}}^{h}+\|(a, \vec{u})\|_{\dot{B}_{2,1}^{s}}^{\ell}\right) d \tau .
\end{aligned}
$$

\footnotetext{
6 The reader may refer to the Appendix for the definition of the paraproduct operator $T$.
} 
Proof As our proof will be essentially based on the results of the previous section, we rescale System (95) as in (36). From the point of view of a priori estimates, this is harmless for the numbers coming into play in the rescaling process are independent of $\mathcal{C}$.

Now as in [12], we localize that (rescaled) system according to Littlewood-Paley decomposition (shortly introduced in the Appendix). Setting $a_{k}:=\dot{\Delta}_{k} a, \vec{u}_{k}:=\dot{\Delta}_{k} \vec{u}$ and so on, we get

$$
\left\{\begin{array}{l}
\partial_{t} a_{k}+\operatorname{div} \vec{u}_{k}=F_{k}^{\prime}, \\
\partial_{t} \vec{u}_{k}-\bar{\mu} \Delta \vec{u}_{k}-(\bar{\lambda}+\bar{\mu}) \nabla \operatorname{div} \vec{u}_{k}+\nabla a_{k}+\nabla \Theta_{k}-\vec{j}_{1, k}=\vec{G}_{k}^{\prime}, \\
\partial_{t} \Theta_{k}-\bar{\kappa} \Delta \Theta_{k}+\eta_{2} \operatorname{div} \vec{u}_{k}+\eta_{3} \Theta_{k}-\eta_{4} j_{0, k}=H_{k}, \\
\partial_{t} j_{0, k}+\eta_{5} j_{0, k}+\eta_{6} \operatorname{div} \vec{j}_{1, k}-\eta_{7} \Theta_{k}=J_{0, k}, \\
\partial_{t} \vec{j}_{1, k}+\eta_{8} \vec{j}_{1, k}+\eta_{6} \nabla j_{0, k}=\vec{J}_{1, k},
\end{array}\right.
$$

with $F_{k}^{\prime}:=F_{k}-\dot{\Delta}_{k}\left(T_{\vec{v}} \cdot \nabla a\right)$ and $\vec{G}_{k}^{\prime}:=\vec{G}_{k}-\dot{\Delta}_{k}\left(T_{\vec{v}} \cdot \nabla \vec{u}\right)$.

Estimates for low frequencies Let

$$
X_{k}:=\left(a_{k}, \vec{u}_{k}, \Theta_{k}, j_{0, k}, j_{1, k}\right)^{T} \quad \text { and } \quad Y_{k}:=\left(F_{k}^{\prime}, \vec{G}_{k}^{\prime}, H_{k}, J_{0, k}, \vec{J}_{1, k}\right)^{T} .
$$

Denoting by $A(D)$ the infinitesimal generator associated to the semi-group corresponding to the (rescaled) System (99), Duhamel's formula yields

$$
X_{k}(t)=e^{A(D) t} X_{k}(0)+\int_{0}^{t} e^{A(D)(t-\tau)} Y_{k}(\tau) \mathrm{d} \tau .
$$

Applying Fourier-Plancherel theorem, following the computations leading to (89), using Remark 3.2 and remembering (94) to handle the incompressible part of $\vec{u}$ and $\vec{j}_{1}$, we get some $k_{0} \in \mathbb{Z}$ such that for all $k \leq k_{0}$,

$$
\left\|X_{k}(t)\right\|_{L^{2}}+2^{2 k} \int_{0}^{t}\left\|X_{k}\right\|_{L^{2}} \mathrm{~d} \tau+\mathcal{C} \int_{0}^{t}\left\|\left(\zeta_{0, k}, \vec{j}_{1, k}\right)\right\|_{L^{2}} \mathrm{~d} \tau \leq C\left(\left\|X_{k}(0)\right\|_{L^{2}}+\int_{0}^{t}\left\|Y_{k}\right\|_{L^{2}} \mathrm{~d} \tau\right) .
$$

Multiplying both sides by $2^{k s}$ and summing up over $k \leq k_{0}$ yields (97).

Estimates for high frequencies Here paralinearization is fundamental, as it allows to avoid the loss of one derivative that may be caused by the convection term in the first equation of (99). Even though the final estimate will be the same for any frequency larger than $2^{k_{0}}$ (where the integer $k_{0}$ is chosen so that $2 \rho_{\ell} \leq 2^{k_{0}}<4 \rho_{\ell}$ ), we have to separate our analysis into two sub-cases corresponding to middle frequencies (i.e., $k_{0} \leq k \leq 1+\log _{2}\left(\rho_{0} \sqrt{\mathcal{C} / \bar{\kappa}}\right)$ ) and $k \geq \log _{2}\left(\rho_{0} \sqrt{\mathcal{C} / \bar{\kappa}}\right)$ because different Lyapunov functionals have been used to obtain (91) and (92).

Let us first focus on middle frequencies : $k_{0} \leq k \leq 1+\log _{2}\left(\rho_{0} \sqrt{\mathcal{C} / \bar{\kappa}}\right)$. Following the analysis of the previous section, we introduce the Lyapunov functional

$$
L_{k}^{2}:=2\left\|a_{k}\right\|_{L^{2}}^{2}+2\left\|\vec{u}_{k}\right\|_{L^{2}}^{2}+\bar{v}^{2}\left\|\nabla a_{k}\right\|_{L^{2}}^{2}+2 \bar{v}\left(\nabla a_{k} \mid u_{k}\right),
$$

and find out that

$$
\begin{aligned}
& \frac{1}{2} \frac{\mathrm{d}}{\mathrm{d} t} L_{k}^{2}+\bar{\mu}\left\|\mathcal{P} u_{k}\right\|_{L^{2}}^{2}+\bar{v}\left\|\left(\nabla a_{k}, \nabla \mathcal{Q} \vec{u}_{k}\right)\right\|_{L^{2}}^{2}=2\left(F_{k}^{\prime} \mid a_{k}\right)+2\left(\left(\vec{j}_{1, k}-\nabla \Theta_{k}+\vec{G}_{k}^{\prime}\right) \mid \vec{u}_{k}\right) \\
& \quad+\left(\bar{v} \nabla F_{k}^{\prime} \mid\left(\bar{v} \nabla a_{k}+\vec{u}_{k}\right)\right)+\left(\bar{v} \nabla a_{k} \mid\left(\vec{j}_{1, k}-\nabla \Theta_{k}+\vec{G}_{k}^{\prime}\right)\right) .
\end{aligned}
$$


The paraconvection terms may be bounded by means of Lemma 4.1 in [12]. More precisely, there exists an integer $N_{0}$ (depending only on the supports of the functions $\varphi$ and $\chi$ involved in the definition of Littlewood-Paley decomposition) and some constant $C$ so that

$$
\begin{aligned}
& \left|\left(\dot{\Delta}_{k}\left(T_{\vec{v}} \cdot \nabla z\right) \mid z_{k}\right)\right| \leq C\|\nabla \vec{v}\|_{L^{\infty}}\left\|z_{k}\right\|_{L^{2}} \sum_{\left|k^{\prime}-k\right| \leq N_{0}}\left\|z_{k^{\prime}}\right\|_{L^{2}} \quad \text { with } z=a, \vec{u}, \\
& \left|\left(\nabla \dot{\Delta}_{k}\left(T_{\vec{v}} \cdot \nabla a\right) \mid \nabla a_{k}\right)\right| \leq C\|\nabla \vec{v}\|_{L^{\infty}}\left\|\nabla a_{k}\right\|_{L^{2}} \sum_{\left|k^{\prime}-k\right| \leq N_{0}}\left\|\nabla a_{k^{\prime}}\right\|_{L^{2}}, \\
& \left|\left(\bar{v} \nabla \dot{\Delta}_{k}\left(T_{\vec{v}} \cdot \nabla a\right) \mid \vec{u}_{k}\right)+\left(\dot{\Delta}_{k}\left(T_{\vec{v}} \cdot \nabla \vec{u}\right) \mid \bar{v} \nabla a_{k}\right)\right| \\
& \leq C\left\|\left(\bar{v} \nabla a_{k}, \vec{u}_{k}\right)\right\|_{L^{2}} \sum_{\left|k^{\prime}-k\right| \leq N_{0}}\left\|\left(\bar{v} \nabla a_{k^{\prime}}, \vec{u}_{k^{\prime}}\right)\right\|_{L^{2}} .
\end{aligned}
$$

Using Bernstein inequality, noticing that

$$
L_{k} \approx\left\|\nabla a_{k}\right\|_{L^{2}}+\left\|\vec{u}_{k}\right\|_{L^{2}} \text { if } k \geq k_{0},
$$

and integrating with respect to time, we thus get for some constant $C=C(\bar{\lambda}, \bar{\mu})$,

$$
\begin{aligned}
& L_{k}(t)+\int_{0}^{t} L_{k} \mathrm{~d} \tau \leq C\left(L_{k}(0)+\int_{0}^{t}\left\|\left(\nabla F_{k}, \vec{G}_{k}\right)\right\|_{L^{2}} \mathrm{~d} \tau\right. \\
& \left.+\sum_{\left|k^{\prime}-k\right| \leq N_{0}} \int_{0}^{t}\|\nabla \vec{v}\|_{L^{\infty}} L_{k^{\prime}} \mathrm{d} \tau+\int_{0}^{t}\left\|\vec{j}_{1, k}-\nabla \Theta_{k}\right\|_{L^{2}} \mathrm{~d} \tau\right) .
\end{aligned}
$$

Of course, from the localized velocity equation, we also gather that

$$
\frac{1}{2} \frac{\mathrm{d}}{\mathrm{d} t}\left\|\vec{u}_{k}\right\|_{L^{2}}^{2}+\bar{\mu}\left\|\nabla \mathcal{P} \vec{u}_{k}\right\|_{L^{2}}^{2}+\bar{v}\left\|\mathcal{Q} \vec{u}_{k}\right\|_{L^{2}}^{2}=\left(\left(\vec{j}_{1, k}-\nabla \Theta_{k}+\vec{G}_{k}^{\prime}\right) \mid \vec{u}_{k}\right)-\left(\nabla a_{k} \mid \vec{u}_{k}\right),
$$

which implies, for some $C=C(\bar{\lambda}, \bar{\mu})$,

$$
\begin{gathered}
\left\|\vec{u}_{k}(t)\right\|_{L^{2}}+2^{2 k} \int_{0}^{t}\left\|\vec{u}_{k}\right\|_{L^{2}} \mathrm{~d} \tau \leq C\left(\int_{0}^{t}\left\|\vec{G}_{k}+\vec{j}_{1, k}-\nabla \Theta_{k}\right\|_{L^{2}} \mathrm{~d} \tau\right. \\
\left.+\sum_{\left|k^{\prime}-k\right| \leq N_{0}} \int_{0}^{t}\|\nabla \vec{v}\|_{L^{\infty}}\left\|\vec{u}_{k^{\prime}}\right\|_{L^{2}} \mathrm{~d} \tau+\int_{0}^{t}\left\|\nabla a_{k}\right\|_{L^{2}} \mathrm{~d} \tau\right) .
\end{gathered}
$$

Therefore combining with (103) and using (102) allows to exhibit the parabolic behavior of $\vec{u}$ : there exists some constant $C=C(\bar{\lambda}, \bar{\mu})$ such that for all $k \geq k_{0}$,

$$
\begin{aligned}
& L_{k}(t)+\int_{0}^{t}\left(\left\|\nabla a_{k}\right\|_{L^{2}}+2^{2 k}\left\|\vec{u}_{k}\right\|_{L^{2}}\right) \mathrm{d} \tau \leq C\left(L_{k}(0)+\int_{0}^{t}\left\|\left(\nabla F_{k}, \vec{G}_{k}\right)\right\|_{L^{2}} \mathrm{~d} \tau\right. \\
& \left.+\sum_{\left|k^{\prime}-k\right| \leq N_{0}} \int_{0}^{t}\|\nabla \vec{v}\|_{L^{\infty}} L_{k^{\prime}} \mathrm{d} \tau+\int_{0}^{t}\left\|\vec{j}_{1, k}-\nabla \Theta_{k}\right\|_{L^{2}} \mathrm{~d} \tau\right) .
\end{aligned}
$$

In order to bound $\Theta_{k}, j_{0, k}$ and $\vec{j}_{1, k}$, we consider the sub-system corresponding to the last three lines of (99), looking at $\eta_{2} \operatorname{div} \vec{u}_{k}$ as a source term. Then we introduce the pseudo-differential operator $B(D):=\eta_{6}^{-1}\left(\eta_{7} \mathrm{Id}+A \eta_{4}(-\Delta)^{-1}\right.$ ) (where the positive number $A$ is chosen as in (79) with $\rho_{h}=\rho_{0} \sqrt{\mathcal{C} / \bar{\kappa}}$ ) and the Lyapunov functional

$$
M_{k}^{2}:=A\left\|\Lambda^{-1} \Theta_{k}\right\|_{L^{2}}^{2}+\left\|\left(j_{0, k}, \vec{j}_{1, k}\right)\right\|_{L^{2}}^{2}-2\left(B(D) \Lambda^{-1} \Theta_{k} \mid \Lambda^{-1} \operatorname{div} \vec{j}_{1, k}\right) .
$$


Following the computations leading to (75), we discover that

$$
\begin{aligned}
\frac{1}{2} & \frac{\mathrm{d}}{\mathrm{d} t} M_{k}^{2}+A \eta_{3}\left\|\Lambda^{-1} \Theta_{k}\right\|_{L^{2}}^{2}+A \bar{\kappa}\left\|\Theta_{k}\right\|_{L^{2}}^{2}+\eta_{5}\left\|j_{0, k}\right\|_{L^{2}}^{2}+\eta_{8}\left\|\vec{j}_{1, k}\right\|_{L^{2}}^{2} \\
& -\left(B(D)\left(\left(\eta_{3}+\eta_{8}\right) \operatorname{Id}+\bar{\kappa}(-\Delta)^{-1}\right) \Lambda^{-1} \Theta_{k} \mid \vec{j}_{1, k}\right)+\eta_{4}\left(B(D)(-\Delta)^{-1} j_{0} \mid \operatorname{div} \vec{j}_{1, k}\right) \\
= & A\left(\Lambda^{-1}\left(H_{k}-\eta_{2} \operatorname{div} \vec{u}_{k}\right) \mid \Lambda^{-1} \Theta_{k}\right)+\left(j_{0, k} \mid J_{0, k}\right)+\left(\vec{j}_{1, k} \mid \vec{J}_{1, k}\right) \\
& -\left(\left(\Lambda^{-1}\left(H_{k}-\eta_{2} \operatorname{div} \vec{u}_{k}\right) \mid B(D) \vec{j}_{1, k}\right)-\left(\vec{J}_{1, k} \mid B(D) \Lambda^{-1} \Theta_{k}\right) .\right.
\end{aligned}
$$

Then mimicking the arguments leading to (81) and using Fourier-Plancherel theorem, we eventually get

$$
\begin{aligned}
& \left\|\left(\Lambda^{-1} \Theta_{k}, j_{0, k}, \vec{j}_{1, k}\right)(t)\right\|_{L^{2}}+\mathcal{C} \int_{0}^{t}\left\|\left(\Lambda^{-1} \Theta_{k}, j_{0, k}, \vec{j}_{1, k}\right)\right\|_{L^{2}} \mathrm{~d} \tau \\
& \quad \lesssim\left\|\left(\Lambda^{-1} \Theta_{k}, j_{0, k}, \vec{j}_{1, k}\right)(0)\right\|_{L^{2}}+\int_{0}^{t}\left\|\left(\Lambda^{-1} H_{k}, J_{0, k}, \vec{J}_{1, k}\right)\right\|_{L^{2}} \mathrm{~d} \tau+\int_{0}^{t}\left\|\vec{u}_{k}\right\|_{L^{2}} \mathrm{~d} \tau .
\end{aligned}
$$

Combining with (104) thus yields for $k_{0} \leq k \leq 1+\log _{2}\left(\rho_{0} \sqrt{\mathcal{C} / \bar{\kappa}}\right)$,

$$
\begin{aligned}
& \left\|\left(\nabla a_{k}, \vec{u}_{k}, \Lambda^{-1} \Theta_{k}, j_{0, k}, \vec{j}_{1, k}\right)(t)\right\|_{L^{2}}+\int_{0}^{t}\left\|\nabla a_{k}\right\|_{L^{2}} \mathrm{~d} \tau+2^{2 k} \int_{0}^{t}\left\|\vec{u}_{k}\right\|_{L^{2}} \mathrm{~d} \tau \\
& \quad+\mathcal{C} \int_{0}^{t}\left\|\left(\Lambda^{-1} \Theta_{k}, j_{0, k}, \vec{j}_{1, k}\right)\right\|_{L^{2}} \mathrm{~d} \tau \lesssim\left\|\left(\nabla a_{k}, \vec{u}_{k}, \Lambda^{-1} \Theta_{k}, j_{0, k}, \vec{j}_{1, k}\right)(0)\right\|_{L^{2}} \\
& \quad+\int_{0}^{t}\left\|\left(\nabla F_{k}, \vec{G}_{k}, \Lambda^{-1} H_{k}, J_{0, k}, \vec{J}_{1, k}\right)\right\|_{L^{2}} \mathrm{~d} \tau+\sum_{\left|k^{\prime}-k\right| \leq N_{0}} \int_{0}^{t}\|\nabla \vec{v}\|_{L^{\infty}}\left\|\left(\nabla a_{k}, \vec{u}_{k}\right)\right\|_{L^{2}} \mathrm{~d} \tau .
\end{aligned}
$$

Let us finally go the case $k>\log _{2}\left(\rho_{0} \sqrt{\mathcal{C} / \bar{\kappa}}\right)$. Then applying an energy method to the equation of $\Lambda^{-1} \Theta_{k}$ yields

$$
\begin{gathered}
\left\|\Lambda^{-1} \Theta_{k}(t)\right\|_{L^{2}}+\bar{\kappa} \int_{0}^{t}\left\|\Theta_{k}\right\|_{L^{2}} \mathrm{~d} \tau \leq\left\|\Lambda^{-1} \Theta_{k}(0)\right\|_{L^{2}} \\
+\int_{0}^{t}\left(\left\|\Lambda^{-1} H_{k}\right\|_{L^{2}}+\eta_{4}\left\|\Lambda^{-1} j_{0, k}\right\|_{L^{2}}+\eta_{2}\left\|\vec{u}_{k}\right\|_{L^{2}}\right) \mathrm{d} \tau,
\end{gathered}
$$

and for the radiative modes, we readily have

$$
\begin{aligned}
& \left\|\left(j_{0, k}, \vec{j}_{1, k}\right)(t)\right\|_{L^{2}}+\min \left(\eta_{5}, \eta_{8}\right) \int_{0}^{t}\left\|\left(j_{0, k}, \vec{j}_{1, k}\right)\right\|_{L^{2}} \mathrm{~d} \tau \leq\left\|\left(j_{0, k}, \vec{j}_{1, k}\right)(0)\right\|_{L^{2}} \\
& \quad+\eta_{7} \int_{0}^{t}\left\|\Theta_{k}\right\|_{L^{2}} \mathrm{~d} \tau+\int_{0}^{t}\left\|\left(J_{0, k}, \vec{J}_{1, k}\right)\right\|_{L^{2}} \mathrm{~d} \tau .
\end{aligned}
$$

Hence combining with (104) and taking $\rho_{0}$ large enough, we get for $k>\log _{2}\left(\rho_{0} \sqrt{\mathcal{C} / \bar{\kappa}}\right)$,

$$
\begin{aligned}
& \left\|\left(\nabla a_{k}, \vec{u}_{k}, \Lambda^{-1} \Theta_{k}, j_{0, k}, \vec{j}_{1, k}\right)(t)\right\|_{L^{2}} \\
& \quad+\int_{0}^{t}\left(\left\|\nabla a_{k}\right\|_{L^{2}}+2^{2 k}\left\|\vec{u}_{k}\right\|_{L^{2}}+2^{k}\left\|\Theta_{k}\right\|_{L^{2}}+\mathcal{C}\left\|\left(j_{0, k}, \vec{j}_{1, k}\right)\right\|_{L^{2}}\right) \mathrm{d} \tau
\end{aligned}
$$




$$
\begin{aligned}
& \lesssim\left\|\left(\nabla a_{k}, \vec{u}_{k}, \Lambda^{-1} \Theta_{k}, j_{0, k}, \vec{j}_{1, k}\right)(0)\right\|_{L^{2}}+\int_{0}^{t}\left(\left\|\left(\nabla F_{k}, \vec{G}_{k}, \Lambda^{-1} H_{k}, J_{0, k}, \vec{J}_{1, k}\right)\right\|_{L^{2}} \mathrm{~d} \tau\right. \\
& \quad+\sum_{\left|k^{\prime}-k\right| \leq N_{0}} \int_{0}^{t}\|\nabla v\|_{L^{\infty}}\left\|\left(\nabla a_{k^{\prime}}, \vec{u}_{k^{\prime}}\right)\right\|_{L^{2}} \mathrm{~d} \tau .
\end{aligned}
$$

Putting together with Inequality (105), multiplying both sides by $2^{k s^{\prime}}$ and summing up over $k \geq k_{0}$ completes the proof of (98). Note that owing to the sum over $k^{\prime}$, there is a small overlap with low frequencies, which explains the presence of the last term of (98).

\section{The well-posedness issue in the critical regularity framework}

This section is mainly devoted to the proof of Theorem 2.3. In passing, we sketch the proof of our local-in-time statement in the critical framework (Theorem 2.2) and justify the nonrelativistic limit pointed out in Corollary 2.1.

Let us first say a few words on the uniqueness issue, which is the consequence of stability estimates in a suitable space. As usual, as a part of System (24) [or (25)] is quasilinear hyperbolic, proving (directly) stability estimates in the solution space $E^{\frac{n}{2}-1}$ is hopeless. The loss of one derivative coming from the density equation induces us to use the larger space $E^{\frac{n}{2}-2}$ (or rather, its local-in-time version).

In high dimension $n \geq 4$ indeed, one can prove stability estimates in $E^{\frac{n}{2}-2}$, just by combining standard hyperbolic and parabolic estimates, and product laws. The proof goes along the lines of that for the nonradiative polytropic compressible Navier-Stokes equations in [7] and does not present any new difficulty (apart from wearisomeness). The case $n=3$ turns out to be critical and one cannot achieve stability estimates in $E^{-\frac{1}{2}}$ by a direct application of hyperbolic and parabolic estimates because some nonlinear terms are not under control. For example, the product of two functions in $\dot{B}_{2,1}^{\frac{1}{2}}\left(\mathbb{R}^{3}\right)$ and $\dot{B}_{2,1}^{-\frac{1}{2}}\left(\mathbb{R}^{3}\right)$, respectively, does not belong to $\dot{B}_{2,1}^{-\frac{3}{2}}\left(\mathbb{R}^{3}\right)$ but to the slightly larger Besov space $\dot{B}_{2, \infty}^{-\frac{3}{2}}\left(\mathbb{R}^{3}\right)$. This obstacle may be overcome by proving stability estimates in a wider space (roughly, Besov spaces $\dot{B}_{2,1}^{s}$ have been changed to $\dot{B}_{2, \infty}^{s}$ in the definition of $E^{-\frac{1}{2}}$ ), and using a logarithmic interpolation inequality. This is just an adaptation of the corresponding proof for nonradiative flows (see [8]).

As for the existence issue, it is very similar to that of the barotropic case (see Subsection 5.1. in [12]). It is only a matter of combining a priori estimates for transport equations (to handle $a$ ), hyperbolic symmetrizable systems with constant coefficients (radiative unknowns) and parabolic equations or systems (for the temperature and velocity). The main difficulty is that the velocity and temperature equations have nonconstant coefficients, depending on $a$ in their leading order, and that $a$ has critical regularity. Exactly as in [12], this may be overcome by splitting $a$ into some (smooth) low-frequency part $\dot{S}_{m} a$, and small high-frequency part (Id $\left.-\dot{S}_{m}\right) a$, treated as a remainder source term. The parameter $m \in \mathbb{Z}$ has to be adjusted conveniently according to the decay of the high frequencies of the initial data $a^{0}$. As presenting the whole proof would be a bit lengthy, and does not require any new idea compared to the barotropic case, we skip the details.

The rest of this section is devoted to the global existence statement of Theorem 2.3. The key is the proof of the global a priori estimates (29) and (30) for smooth solutions to (25). As those estimates are uniquely based on energy arguments, Friedrichs method (used in, 
e.g., Chap. 10 of [1] in the nonradiative case) allows to construct a sequence of approximate smooth solutions satisfying exactly the same estimates.

The proof of global a priori estimates for a smooth enough solution $\left(a, \vec{u}, \Theta, j_{0}, \vec{j}_{1}\right)$ to System (25) relies on Proposition 4.1 with $s=s^{\prime}=\frac{n}{2}-1, \vec{v}=\vec{u}$, and source terms ${ }^{7}$

$$
\begin{aligned}
F:= & T_{\vec{u}} \cdot \nabla a-\vec{u} \cdot \nabla a-a \operatorname{div} \vec{u}, \\
\vec{G}:= & T_{\vec{u}} \cdot \nabla \vec{u}-\vec{u} \cdot \nabla \vec{u}+\frac{1}{1+a}(\operatorname{div}(2 \mu D \vec{u})+\nabla(\lambda \operatorname{div} \vec{u})-\underline{\mathcal{A}} \vec{u}) \\
& +\left(\pi_{0}^{\prime}(1)-\frac{\pi_{0}^{\prime}(1+a)}{1+a}+\pi_{1}^{\prime}(1)-(1+\Theta) \frac{\pi_{1}^{\prime}(1+a)}{1+a}\right) \nabla a \\
& +\left(\pi_{1}(1)-\frac{\pi_{1}(1+a)}{1+a}\right) \nabla \Theta-\left(\frac{\sigma_{a}+\sigma_{s}}{n}\right)\left(\frac{a}{1+a}\right) \vec{j}_{1}, \\
H:= & -\vec{u} \cdot \nabla \Theta+\frac{1}{1+a} \operatorname{div}((\kappa-\underline{\kappa}) \nabla \Theta)+\left(\pi_{1}(1)-(1+\Theta) \frac{\pi_{1}(1+a)}{1+a}\right) \operatorname{div} \vec{u} \\
& +\frac{1}{n}\left(\frac{\sigma_{a}+\sigma_{s}}{1+a}\right) \vec{j}_{1} \cdot \vec{u}-\mathcal{C} \sigma_{a} \frac{a}{1+a}\left(\underline{\alpha}^{\prime} \Theta-j_{0}\right) \\
& +\frac{1}{1+a}\left(2 \underline{\mu} D \vec{u}: D \vec{u}+\underline{\lambda}(\operatorname{div} \vec{u})^{2}\right), \\
J_{0}:= & 0 \text { and } \vec{J}_{1}:=\mathcal{C}\left(\sigma_{s}-\underline{\sigma}_{s}\right) \vec{j}_{1} .
\end{aligned}
$$

Denoting

$$
\begin{aligned}
X(t):= & \|(a, \Theta)(t)\|_{\dot{B}_{2,1}^{\frac{n}{2}-1}}^{\ell}+\|a(t)\|_{\dot{B}_{2,1}^{\frac{n}{2}}}^{h}+\|\Theta(t)\|_{\dot{B}_{2,1}^{\frac{n}{2}-2}}^{h}+\left\|\left(\vec{u}, j_{0}, \vec{j}_{1}\right)(t)\right\|_{\dot{B}_{2,1}^{\frac{n}{2}-1}} \\
& +\int_{0}^{t}\left(\left\|\left(a, \Theta, j_{0}, \vec{j}_{1}\right)\right\|_{\dot{B}_{2,1}^{\frac{n}{2}+1}}^{\ell}+\|\vec{u}\|_{\dot{B}_{2,1}^{2}}^{\frac{n}{2}+1}+\|(a, \Theta)\|_{\dot{B}_{2,1}^{\frac{n}{2}}}^{h}\right) \mathrm{d} \tau \\
& +\mathcal{C} \int_{0}^{t}\left(\left\|\vec{j}_{1}\right\|_{\dot{B}_{2,1}^{\frac{n}{2}-1}}+\left\|\zeta_{0}\right\|_{\dot{B}_{2,1}^{\frac{n}{2}-1}}^{\ell}+\left\|j_{0}\right\|_{\dot{B}_{2,1}^{\frac{n}{2}-1}}^{h}+\|\Theta\|_{\dot{B}_{2,1}^{\frac{n}{2}-2}}^{h}\right) \mathrm{d} \tau
\end{aligned}
$$

we get

$$
\begin{aligned}
& X(t) \lesssim X(0)+\int_{0}^{t}\|\nabla \vec{u}\|_{L^{\infty}} X \mathrm{~d} \tau+\int_{0}^{t}\left(\left\|J_{0}\right\|_{\dot{B}_{2,1}^{\frac{n}{2}-1}}+\left\|\vec{J}_{1}\right\|_{\dot{B}_{2,1}^{\frac{n}{2}-1}}\right) \mathrm{d} \tau \\
& +\int_{0}^{t}\left\|\left(F-T_{\vec{u}} \cdot \nabla a, \vec{G}-T_{\vec{u}} \cdot \nabla \vec{u}, H\right)\right\|_{\dot{B}_{2,1}^{\frac{n}{2}-1}}^{\ell} \\
& +\int_{0}^{t}\left(\|F\|_{\dot{B}_{2,1}^{\frac{n}{2}}}^{h}+\|\vec{G}\|_{\dot{B}_{2,1}^{\frac{n}{2}-1}}^{h}+\|H\|_{\dot{B}_{2,1}^{\frac{n}{2}-2}}^{h}\right) \mathrm{d} \tau .
\end{aligned}
$$

Note that $\|\nabla \vec{u}\|_{L^{\infty}} \lesssim\|\vec{u}\|_{\dot{B}_{2,1}^{2}+1}$. Therefore in order to close the estimates globally for small $X(0)$ (that is small data), it suffices to bound the last three integrals in (108) by $C X^{2}(t)$. For that, we shall use repeatedly the fact that

$$
\|a\|_{\dot{B}_{2,1}^{\frac{n}{2}-1}}^{\ell}+\|a\|_{\dot{B}_{2,1}^{\frac{n}{2}}}^{h} \approx\|a\|_{\dot{B}_{2,1}^{\frac{n}{2}-1} \cap \dot{B}_{2,1}^{\frac{n}{2}}} \text { and }\|\Theta\|_{\dot{B}_{2,1}^{\frac{n}{2}-1}}^{\ell}+\|\Theta\|_{\dot{B}_{2,1}^{\frac{n}{2}-2}}^{h} \approx\|\Theta\|_{\dot{B}_{2,1}^{\frac{n}{2}-2}+\dot{B}_{2,1}^{\frac{n}{2}-1}}
$$

Another useful property is that, owing to interpolation and Hölder inequality,

$$
\|a\|_{L_{t}^{2}\left(\dot{B}_{2,1}^{\frac{n}{2}}\right)} \lesssim X(t) .
$$

7 To simplify the presentation, we assume that $\operatorname{Ma}=\operatorname{Pr}=\operatorname{Re}=\mathcal{L}=\mathcal{L}_{s}=1$. 
We shall finally assume that

$$
\|a\|_{L^{\infty}\left(\mathbb{R}^{+} \times \mathbb{R}^{n}\right)} \text { is small enough, }
$$

a property that will be used implicitly whenever composition estimates are applied. Of course, as we will get eventually that $\|a\|_{L^{\infty}\left(\dot{B}_{2,1}^{\frac{n}{2}}\right)}$ is small, and as $\dot{B}_{2,1}^{\frac{n}{2}}$ is embedded in $L^{\infty}$, Assumption (111) may be justified a posteriori.

Step 1. Estimates for $F$ and $F-T_{\vec{u}} \cdot \nabla a$ Because $F-T_{\vec{u}} \cdot \nabla a=-\vec{u} \cdot \nabla a-a \operatorname{div} \vec{u}$ and the product maps $\dot{B}_{2,1}^{\frac{n}{2}} \times \dot{B}_{2,1}^{\frac{n}{2}-1}$ in $\dot{B}_{2,1}^{\frac{n}{2}-1}$, we readily have

$$
\left\|F-T_{\vec{u}} \cdot \nabla a\right\|_{\dot{B}_{2,1}^{\frac{n}{2}-1}} \lesssim\|\vec{u}\|_{\dot{B}_{2,1}^{\frac{n}{2}}}\|\nabla a\|_{\dot{B}_{2,1}^{\frac{n}{2}-1}} .
$$

Similarly, Lemma 7.2 implies that

$$
\|F\|_{\dot{B}_{2,1}^{\frac{n}{2}}} \lesssim\|\vec{u}\|_{\dot{B}_{2,1}^{\frac{n}{2}+1}}\|a\|_{\dot{B}_{2,1}^{\frac{n}{2}}}
$$

Therefore for all $t>0$,

$$
\int_{0}^{t}\left(\left\|F-T_{\vec{u}} \cdot \nabla a\right\|_{\dot{B}_{2,1}^{\frac{n}{2}-1}}^{\ell}+\|F\|_{\dot{B}_{2,1}^{\frac{n}{2}}}^{h}\right) \mathrm{d} \tau \lesssim X^{2}(t) .
$$

Step 2. Estimates for $\vec{G}$ and $\vec{G}-T_{\vec{u}} \cdot \nabla \vec{u}$ Arguing as for $F$, we get

$$
\left\|\vec{u} \cdot \nabla \vec{u}-T_{\vec{u}} \cdot \nabla \vec{u}\right\|_{\dot{B}_{2,1}^{\frac{n}{2}-1}}+\|\vec{u} \cdot \nabla \vec{u}\|_{\dot{B}_{2,1}^{\frac{n}{2}-1}} \lesssim\|\vec{u}\|_{\dot{B}_{2,1}^{\frac{n}{2}}}^{2} .
$$

Next, we observe that

$$
\frac{1}{1+a} \operatorname{div}(\mu D \vec{u})-\underline{\mu} \operatorname{div} D \vec{u}=\frac{1}{1+a} \operatorname{div}((\mu-\underline{\mu}) D \vec{u})-\frac{a}{1+a} \underline{\mu} \operatorname{div} D \vec{u}
$$

and a similar equality for $\frac{1}{1+a} \nabla(\lambda \operatorname{div} \vec{u})-\underline{\lambda} \nabla \operatorname{div} \vec{u}$. Hence combining Lemmas 7.3 and 7.4 yields

$$
\left\|\frac{1}{1+a}(\operatorname{div}(2 \mu D \vec{u})+\nabla(\lambda \operatorname{div} \vec{u})-\underline{\mathcal{A}} \vec{u})\right\|_{\dot{B}_{2,1}^{\frac{n}{2}-1}} \lesssim\left(1+\|a\|_{\dot{B}_{2,1}^{\frac{n}{2}}}\right)\|a\|_{\dot{B}_{2,1}^{\frac{n}{2}}\|\nabla \vec{u}\|_{\dot{B}_{2,1}^{\frac{n}{2}}}} .
$$

The terms of $\vec{G}$ involving the pressure may be written

$$
\pi_{2}(a) \nabla a+\pi_{3}(a) \Theta \nabla a+\pi_{4}(a) \nabla \Theta \text { with } \pi_{2}(0)=\pi_{4}(0)=0 .
$$

Combining Lemma 7.4 and product estimates, we get

$$
\left\|\pi_{2}(a) \nabla a\right\|_{\dot{B}_{2,1}^{\frac{n}{2}-1}} \lesssim\|a\|_{\dot{B}_{2,1}^{\frac{n}{2}}}\|\nabla a\|_{\dot{B}_{2,1}^{\frac{n}{2}-1}} .
$$

For the other two terms, we decompose $\Theta$ into $\Theta^{\ell}+\Theta^{h}$ so as to write that

$$
\pi_{3}(a) \Theta \nabla a=\pi_{3}(a) \Theta^{\ell} \nabla a+\pi_{3}(a) \Theta^{h} \nabla a \text { and } \pi_{4}(a) \nabla \Theta=\pi_{4}(a) \nabla \Theta^{\ell}+\pi_{4}(a) \nabla \Theta^{h} .
$$


We thus get

$$
\begin{aligned}
&\left\|\pi_{3}(a) \Theta \nabla a\right\|_{L_{t}^{1}\left(\dot{B}_{2,1}^{\frac{n}{2}-1}\right)} \lesssim\left(1+\|a\|_{L_{t}^{\infty}\left(\dot{B}_{2,1}^{\frac{n}{2}}\right)}\right)\left(\left\|\Theta^{\ell}\right\|_{L_{t}^{2}\left(\dot{B}_{2,1}^{\frac{n}{2}}\right)}\|\nabla a\|_{L_{t}^{2}\left(\dot{B}_{2,1}^{\frac{n}{2}-1}\right)}\right. \\
&\left.+\left\|\Theta^{h}\right\|_{L_{t}^{1}\left(\dot{B}_{2,1}^{\frac{n}{2}}\right)}\|\nabla a\|_{L_{t}^{\infty}\left(\dot{B}_{2,1}^{\frac{n}{2}-1}\right)}\right), \\
&\left\|\pi_{4}(a) \nabla \Theta\right\|_{L_{t}^{1}\left(\dot{B}_{2,1}^{\frac{n}{2}-1}\right)} \lesssim\|a\|_{L_{t}^{2}\left(\dot{B}_{2,1}^{\frac{n}{2}}\right)}\left\|\nabla \Theta^{\ell}\right\|_{L^{2}\left(\dot{B}_{2,1}^{\frac{n}{2}-1}\right)}+\|a\|_{L_{t}^{\infty}\left(\dot{B}_{2,1}^{\frac{n}{2}}\right)}\left\|\nabla \Theta^{h}\right\|_{L_{t}^{1}\left(\dot{B}_{2,1}^{\frac{n}{2}-1}\right)} .
\end{aligned}
$$

The last term of $\vec{G}$ reads $\pi_{5}(a) \vec{j}_{1}$ for some smooth function $\pi_{5}$ vanishing at 0 . Hence we just have

$$
\left\|\pi_{5}(a) \vec{j}_{1}\right\|_{L_{t}^{1}\left(\dot{B}_{2,1}^{\frac{n}{2}-1}\right)} \lesssim\|a\|_{L_{t}^{\infty}\left(\dot{B}_{2,1}^{\frac{n}{2}}\right)}\left\|\vec{j}_{1}\right\|_{L_{t}^{1}\left(\dot{B}_{2,1}^{\frac{n}{2}-1}\right)} .
$$

So finally, putting together all the inequalities of this step, we conclude that for all $t>0$,

$$
\int_{0}^{t}\left(\left\|\vec{G}-T_{\vec{u}} \cdot \nabla a\right\|_{\dot{B}_{2,1}^{\frac{n}{2}-1}}+\|\vec{G}\|_{\dot{B}_{2,1}^{\frac{n}{2}-1}}\right) \mathrm{d} \tau \lesssim X^{2}(t) .
$$

Step 3. Estimates for $H$ According to (109), it suffices to bound $H$ in $L^{1}\left(\mathbb{R}^{+} ; \dot{B}_{2,1}^{\frac{n}{2}-1}+\dot{B}_{2,1}^{\frac{n}{2}-2}\right)$. Now decomposing $\Theta$ in $\Theta^{\ell}+\Theta^{h}$, and using product laws in Besov spaces, we get if $n \geq 3$,

$$
\|\vec{u} \cdot \nabla \Theta\|_{L_{t}^{1}\left(\dot{B}_{2,1}^{\frac{n}{2}-1}+\dot{B}_{2,1}^{\frac{n}{2}-2}\right)} \lesssim\|\vec{u}\|_{L_{t}^{2}\left(\dot{B}_{2,1}^{\frac{n}{2}}\right)}\left(\left\|\nabla \Theta^{\ell}\right\|_{L_{t}^{2}\left(\dot{B}_{2,1}^{\frac{n}{2}-1}\right)}+\left\|\nabla \Theta^{h}\right\|_{L_{t}^{2}\left(\dot{B}_{2,1}^{\frac{n}{2}-2}\right)}\right) .
$$

Next, using Lemma 7.4,

$$
\left\|\frac{1}{1+a} \operatorname{div}((\kappa-\underline{\kappa}) \nabla \Theta)\right\|_{L_{t}^{1}\left(\dot{B}_{2,1}^{\frac{n}{2}-1}+\dot{B}_{2,1}^{\frac{n}{2}-2}\right)} \lesssim\|a\|_{L_{t}^{\infty}\left(\dot{B}_{2,1}^{\frac{n}{2}}\right)}\left(\left\|\nabla \Theta^{\ell}\right\|_{L_{t}^{1}\left(\dot{B}_{2,1}^{\frac{n}{2}}\right)}+\left\|\nabla \Theta^{h}\right\|_{L_{t}^{1}\left(\dot{B}_{2,1}^{\frac{n}{2}-1}\right)}\right) .
$$

The term involving the pressure may be written

$$
\left(\pi_{1}(1)-(1+\Theta) \frac{\pi_{1}(1+a)}{1+a}\right) \operatorname{div} \vec{u}=\left(\pi_{6}(a)+\pi_{7}(a) \Theta\right) \operatorname{div} \vec{u} \text { with } \pi_{6}(0)=0 .
$$

Now we have

$$
\begin{aligned}
& \left\|\pi_{6}(a) \operatorname{div} \vec{u}\right\|_{L_{t}^{1}\left(\dot{B}_{2,1}^{\frac{n}{2}-1}\right)} \lesssim\|a\|_{L_{t}^{2}\left(\dot{B}_{2,1}^{\frac{n}{2}}\right)}\|\operatorname{div} \vec{u}\|_{L_{t}^{2}\left(\dot{B}_{2,1}^{\frac{n}{2}-1}\right)}, \\
& \left\|\pi_{7}(a) \Theta \operatorname{div} \vec{u}\right\|_{L_{t}^{1}\left(\dot{B}_{2,1}^{\frac{n}{2}-1}+\dot{B}_{2,1}^{\frac{n}{2}-2}\right)} \lesssim\left(1+\|a\|_{L_{t}^{\infty}\left(\dot{B}_{2,1}^{\frac{n}{2}}\right)}\right)\|\operatorname{div} \vec{u}\|_{L_{t}^{2}\left(\dot{B}_{2,1}^{\frac{n}{2}-1}\right)}\left(\left\|\Theta^{\ell}\right\|_{L_{t}^{2}\left(\dot{B}_{2,1}^{\frac{n}{2}}\right)}+\left\|\Theta^{h}\right\|_{L_{t}^{2}\left(\dot{B}_{2,1}^{\frac{n}{2}-1}\right)}\right) \text {. }
\end{aligned}
$$

The next term reads $\pi_{8}(a) \vec{j}_{1} \cdot \vec{u}$ with $\pi_{8}$ a smooth function of $a$. We thus get

$$
\left\|\pi_{8}(a) \vec{j}_{1} \cdot \vec{u}\right\|_{L_{t}^{1}\left(\dot{B}_{2,1}^{\frac{n}{2}-1}\right)} \lesssim\left(1+\|a\|_{L_{t}^{\infty}\left(\dot{B}_{2,1}^{\frac{n}{2}}\right)}\|\vec{u}\|_{L_{t}^{2}\left(\dot{B}_{2,1}^{\frac{n}{2}}\right)}\left\|\vec{j}_{1}\right\|_{L_{t}^{2}\left(\dot{B}_{2,1}^{\frac{n}{2}-1}\right)} .\right.
$$

The last but one term reads $\mathcal{C} \pi_{9}(a)\left(j_{0}-\underline{\alpha}^{\prime} \Theta\right)$ for some function $\pi_{9}$ vanishing at 0 . To bound it, it is crucial to exploit the fact that $\zeta_{0}^{\ell}$ and $\Theta^{h}$ satisfy fast decay properties. More precisely, remembering the definition of $\zeta_{0}$, we have the decomposition

$$
\mathcal{C} \pi_{9}(a)\left(j_{0}-\underline{\alpha}^{\prime} \Theta\right)=\pi_{9}(a)\left(\mathcal{C}\left(\zeta_{0}^{\ell}+j_{0}^{h}-\underline{\alpha}^{\prime} \Theta^{h}\right)+\frac{\underline{\alpha}_{2} \underline{\alpha}^{\prime}}{\underline{\alpha}_{1} \underline{\sigma}_{a}\left(1+\underline{\alpha}^{\prime}\right)} \operatorname{div} \vec{u}^{\ell}\right) .
$$


Now we have

$$
\begin{aligned}
& \mathcal{C}\left\|\pi_{9}(a)\left(\zeta_{0}^{\ell}+j_{0}^{h}\right)\right\|_{L_{t}^{1}\left(\dot{B}_{2,1}^{\frac{n}{2}-1}\right)} \lesssim \mathcal{C}\left(\left\|\zeta_{0}\right\|_{L_{t}^{1}\left(\dot{B}_{2,1}^{\frac{n}{2}-1}\right)}^{\ell}+\left\|j_{0}\right\|_{L_{t}^{1}\left(\dot{B}_{2,1}^{\frac{n}{2}-1}\right)}^{h}\|a\|_{L_{t}^{\infty}\left(\dot{B}_{2,1}^{\frac{n}{2}}\right)} \lesssim X^{2}(t),\right. \\
& \mathcal{C}\left\|\pi_{9}(a) \Theta^{h}\right\|_{L_{t}^{1}\left(\dot{B}_{2,1}^{\frac{n}{2}-2}\right)} \lesssim \mathcal{C}\left\|\Theta_{L_{t}^{1}\left(\dot{B}_{2,1}^{\frac{n}{2}-2}\right)}^{h}\right\| a \|_{L_{t}^{\infty}\left(\dot{B}_{2,1}^{\frac{n}{2}}\right)} \lesssim X^{2}(t), \\
& \left\|\pi_{9}(a) \operatorname{div} \vec{u}^{\ell}\right\|_{L_{t}^{1}\left(\dot{B}_{2,1}^{\frac{n}{2}-1}\right)} \lesssim\|a\|_{L_{t}^{2}\left(\dot{B}_{2,1}^{\frac{n}{2}}\right)}\|\operatorname{div} \vec{u}\|_{L_{t}^{\ell}\left(\dot{B}_{2,1}^{\frac{n}{2}-1}\right)}^{\ell} \lesssim X^{2}(t) .
\end{aligned}
$$

Finally,

$$
\left\|\frac{1}{1+a}\left(2 \mu D \vec{u}: D \vec{u}+\lambda(\operatorname{div} \vec{u})^{2}\right)\right\|_{\dot{B}_{2,1}^{\frac{n}{2}-2}} \lesssim\left(1+\|a\|_{\dot{B}_{2,1}^{\frac{n}{2}}}\right)\|\nabla u\|_{\dot{B}_{2,1}^{\frac{n}{2}-1}}^{2}
$$

Combining all the above inequalities, we conclude that for all $t \in \mathbb{R}^{+}$,

$$
\int_{0}^{t}\|H\|_{\dot{B}_{2,1}^{2}-1}^{\frac{n}{2}-1} \dot{B}_{2,1}^{\frac{n}{2}-2} \mathrm{~d} \tau \lesssim X^{2}(t) .
$$

Step 4. Estimates for $\vec{J}_{1}$ Because $\vec{J}_{1}$ has the same form as the last term of $\vec{G}$, it may be bounded exactly as in the second step:

$$
\int_{0}^{t}\left\|\vec{J}_{1}\right\|_{\dot{B}_{2,1}^{\frac{n}{2}-1}} \mathrm{~d} \tau \lesssim \mathcal{C}\left\|\vec{j}_{1}\right\|_{L_{t}^{1}\left(\dot{B}_{2,1}^{\frac{n}{2}-1}\right)}\|a\|_{L_{t}^{\infty}\left(\dot{B}_{2,1}^{\frac{n}{2}}\right)} \lesssim X^{2}(t)
$$

Step 5. Conclusion Plugging inequalities (112), (113), (114) and (115) in (108), we get for a constant $C$ depending only on the coefficients of the system, and for all $t \in \mathbb{R}^{+}$,

$$
X(t) \leq C\left(X(0)+X^{2}(t)\right)
$$

which allows to close the estimates for all time, if $X(0)$ is small enough.

For the sake of completeness, we here sketch the proof of Corollary 2.1 (for more details, the reader may refer to our recent papers $[13,14]$ where very similar arguments are used to justify weak convergence in different asymptotics). One can argue as follows:

1. The uniform estimate provided by (29) ensures that, up to extraction, we have

$$
\left(a^{\varepsilon}, \vec{u}^{\varepsilon}, \Theta^{\varepsilon}, j_{0}^{\varepsilon}, \vec{j}_{1}^{\varepsilon}\right) \rightarrow\left(a, \vec{u}, \Theta, j_{0}, \vec{j}_{1}\right)
$$

for the weak $*$ topology associated to the space $E^{\frac{n}{2}-1}$. Standard (omitted) arguments ensure that the limit solution $\left(a, \vec{u}, \Theta, j_{0}, \vec{j}_{1}\right)$ belongs to the superspace $E_{w}^{\frac{n}{2}-1}$ of $E^{\frac{n}{2}-1}$, where strong time continuity has been replaced by weak continuity.

2. Inequality (30) ensures that $\vec{j}_{1}^{\varepsilon} \rightarrow \overrightarrow{0}$ in $L^{1}\left(\mathbb{R}_{+} ; \dot{B}_{2,1}^{\frac{n}{2}-1}\right)$. From the last equation of (25), we thus gather that $\nabla j_{0} \equiv 0$. Because $j_{0}$ is in $L^{\infty}\left(\mathbb{R}_{+} ; \dot{B}_{2,1}^{\frac{n}{2}-1}\right)$ and thus tends weakly to 0 at infinity, we conclude that $j_{0} \equiv 0$.

3. Inequality (30) also implies that $\Theta_{0}^{h} \equiv 0$ and that $\zeta_{0, \varepsilon}^{\ell} \rightarrow 0$. As $j_{0}^{\ell} \equiv 0$ and

$$
\zeta_{0, \varepsilon}=j_{0, \varepsilon}-\underline{\alpha}^{\prime} \Theta_{\varepsilon}-\varepsilon \frac{\underline{\alpha}_{2} \underline{\alpha}^{\prime}}{\mathcal{L} \underline{\alpha}_{1} \underline{\sigma_{a}}\left(1+P r^{-1} \underline{\alpha}^{\prime}\right)} \operatorname{div} \vec{u}^{\varepsilon} \rightarrow j_{0}-\underline{\alpha}^{\prime} \Theta,
$$

this implies that $\Theta^{\ell} \equiv 0$. We thus have $\Theta \equiv 0$. 
4. What we proved hitherto already ensures that the last term of the velocity equation of (25) goes (strongly) to 0 in $L^{1}\left(\mathbb{R}_{+} ; \dot{B}_{2,1}^{\frac{n}{2}-1}\right)$. To pass to the limit in the other terms of the first two equations of (25), we need some compactness. The easiest way to achieve it, is to bound $\partial_{t} a^{\varepsilon}$ and $\partial_{t} \vec{u}^{\varepsilon}$ in some suitable space. Taking advantage of the equations for $a^{\varepsilon}$ and $\vec{u}^{\varepsilon}$, of the uniform bounds provided by (29) and of product laws in Besov spaces, one can show that $\left(\partial_{t} a^{\varepsilon}\right)$ and $\left(\partial_{t} \vec{u}^{\varepsilon}\right)$ are bounded in $L_{l o c}^{2}\left(\mathbb{R}_{+} ; \dot{B}_{2,1}^{\frac{n}{2}-1}\right)$ and $L_{l o c}^{2}\left(\mathbb{R}_{+} ; \dot{B}_{2,1}^{\frac{n}{2}-2}\right)$, respectively. Using compact embedding in Besov spaces, one can conclude to strong convergence results in good enough norms so as to pass to the limit in all the nonlinear terms. Consequently, $(a, \vec{u})$ satisfies (32).

5. Because (32) admits a unique solution in the space given by Corollary 2.1 (up to replacing strong continuity by weak continuity), $(a, \vec{u})$ is uniquely determined, and thus strongly continuous in time. We conclude that all limits of subsequences of $\left(a^{\varepsilon}, \vec{u}^{\varepsilon}, \Theta^{\varepsilon}, j_{0}^{\varepsilon}, \vec{j}_{1}^{\varepsilon}\right)$ are the same. Hence the whole family converges to $(a, \vec{u}, 0,0, \overrightarrow{0})$.

\section{Local existence for general large data}

Given some reference constant positive temperature $\bar{\vartheta}$, we want to establish the local well posedness of the following system governing the evolution of $\varrho, \vec{u}, \Theta:=\vartheta-\bar{\vartheta}, j_{0}:=I_{0}-b(\bar{\vartheta})$ and $\vec{j}_{1}:=\vec{I}_{1}$ :

$$
\left\{\begin{array}{l}
\partial_{t} \varrho+\operatorname{div}(\varrho \vec{u})=0, \\
\varrho\left(\partial_{t} \vec{u}+\vec{u} \cdot \nabla \vec{u}\right)+\nabla p-\operatorname{div}(2 \mu D \vec{u}+\lambda \operatorname{div} \vec{u} \operatorname{Id})=\left(\frac{\sigma_{a}+\sigma_{s}}{n}\right) \vec{j}_{1}, \\
C_{v} \varrho\left(\partial_{t} \Theta+\vec{u} \cdot \nabla \Theta\right)-\operatorname{div}(\kappa \nabla \Theta)=2 \mu D \vec{u}: D \vec{u}+\lambda(\operatorname{div} \vec{u})^{2} \\
\quad-\vartheta \partial_{\vartheta} p \operatorname{div} \vec{u}+\sigma_{a}\left(j_{0}+b(\bar{\vartheta})-b(\vartheta)\right)+\left(\frac{\sigma_{a}+\sigma_{s}}{n}\right) \overrightarrow{j_{1}} \cdot \vec{u}, \\
\partial_{t} j_{0}+\frac{1}{n} \operatorname{div} \vec{j}_{1}=\sigma_{a}\left(b(\vartheta)-b(\bar{\vartheta})-j_{0}\right), \\
\partial_{t} \vec{j}_{1}+\nabla j_{0}+\left(\sigma_{a}+\sigma_{s}\right) \vec{j}_{1}=\overrightarrow{0} .
\end{array}\right.
$$

Recall that the pressure function is given by (9). Here in contrast with the previous section, all the coefficients are allowed to depend (smoothly) on both $\varrho$ and $\vartheta$ provided the positivity condition (22) is fulfilled, and the distribution function $b$ may be anything (if smooth of course).

The rest of this section is devoted to the proof of Theorem 2.1. Given that a lot of regularity is available, proving uniqueness is not a big issue, and is thus skipped. As regards the proof of local existence, it may be worked out from the a priori estimates for (116) that we shall derive below (use, e.g., a Friedrichs scheme, or an iterative method based on a linearization of the system). Those estimates will be obtained by combining results on linear equations (see the three propositions just below) and nonlinear inequalities stated in the Appendix.

More precisely, estimating the density will rely on the following proposition (see, e.g., Th. 3.14 in [1]):

\section{Proposition 6.1 Let a satisfy the transport equation}

$$
\partial_{t} a+\vec{v} \cdot \nabla a=f .
$$

Then for any $s \in\left(-\frac{n}{2}, \frac{n}{2}+1\right]$ there exists a constant $C$ so that for all $t \geq 0$,

$$
\|a(t)\|_{B_{2,1}^{s}} \leq e^{C \int_{0}^{t}\|\nabla \vec{v}\|{ }_{B_{2,1} \frac{n}{2}} \mathrm{~d} \tau}\left(\|a(0)\|_{B_{2,1}^{s}}+\int_{0}^{t} e^{-C \int_{0}^{\tau}\|\nabla \vec{v}\|_{B_{2,1} \frac{n}{2}} d \tau^{\prime}}\|f(\tau)\|_{B_{2,1}^{s}} d \tau\right) .
$$


The estimates for the velocity and temperature equations will be based on the following statement that has been proved in [11] for homogeneous Besov norms ${ }^{8}$.

Proposition 6.2 Let $-\frac{n}{2}<s \leq \frac{n}{2}$ and $u:[0, T] \times \mathbb{R}^{n} \rightarrow \mathbb{R}$ satisfying

$$
\partial_{t} u-a \operatorname{div}(b \nabla u)=f,\left.\quad u\right|_{t=0}=u^{0}
$$

for some functions $a$ and $b$ such that

$$
\alpha:=\inf _{(t, x) \in[0, T] \times \mathbb{R}^{n}}(a b)(t, x)>0 .
$$

Then there exist $\kappa=\kappa(s, n)$ and $C=C(s, n)$ such that for all $t \in[0, T]$,

$$
\begin{gathered}
\|u(t)\|_{B_{2,1}^{s}}+\kappa \alpha \int_{0}^{t}\|u\|_{B_{2,1}^{s+2}} d \tau \leq\left\|u^{0}\right\|_{B_{2,1}^{s}}+\int_{0}^{t}\|f\|_{B_{2,1}^{s}} \mathrm{~d} \tau \\
+C \int_{0}^{t}\left(\alpha+\alpha^{-1}\|b \nabla a, a \nabla b\|_{B_{2,1}^{2}}^{2}\right)\|u\|_{B_{2,1}^{s}} d \tau .
\end{gathered}
$$

Similarly, if $\vec{v}:[0, T] \times \mathbb{R}^{n} \rightarrow \mathbb{R}^{n}$ satisfies

$$
\partial_{t} \vec{v}-2 a \operatorname{div}(\mu D \vec{v})-b \nabla(\lambda \operatorname{div} \vec{v})=\vec{g},\left.\quad \vec{v}\right|_{t=0}=\vec{v}^{0}
$$

with

$$
\alpha:=\min \left(\inf _{(t, x) \in[0, T] \times \mathbb{R}^{n}}(a \mu)(t, x), \inf _{t, x}(2 a \mu+b \lambda)(t, x)\right)>0
$$

then there exist $\kappa=\kappa(s, n)$ and $C=C(s, n)$ such that

$$
\begin{aligned}
& \|\vec{v}(t)\|_{B_{2,1}^{s}}+\kappa \alpha \int_{0}^{t}\|\vec{v}\|_{B_{2,1}^{s+2}} d \tau \leq\left\|\vec{v}^{0}\right\|_{B_{2,1}^{s}}+\int_{0}^{t}\|\vec{g}\|_{B_{2,1}^{s}} d \tau \\
& \quad+C \int_{0}^{t}\left(\alpha+\alpha^{-1}\|\mu \nabla a, a \nabla \mu, \lambda \nabla b, b \nabla \lambda\|_{B_{2,1}^{\frac{n}{2}}}^{2}\|\vec{v}\|_{B_{2,1}^{s}} d \tau .\right.
\end{aligned}
$$

Finally, the radiative modes $j_{0}$ and $\vec{j}_{1}$ will be handled thanks to the obvious following result, in the spirit of [12], page 189:

Proposition 6.3 Let $\left(j_{0}, \vec{j}_{1}\right):[0, T] \times \mathbb{R}^{n} \rightarrow \mathbb{R} \times \mathbb{R}^{n}$ satisfy

$$
\left\{\begin{array}{l}
\partial_{t} j_{0}+\frac{1}{n} \operatorname{div} \vec{j}_{1}=J_{0} \\
\partial_{t} \vec{j}_{1}+\nabla j_{0}=\vec{J}_{1}
\end{array}\right.
$$

Then for any $s \in \mathbb{R}$ and $t \in[0, T]$, we have

$$
\left\|\left(j_{0}, \frac{1}{\sqrt{n}} \vec{j}_{1}\right)(t)\right\|_{B_{2,1}^{s}} \leq\left\|\left(j_{0}, \frac{1}{\sqrt{n}} \vec{j}_{1}\right)(0)\right\|_{B_{2,1}^{s}}+\int_{0}^{t}\left\|\left(J_{0}, \frac{1}{\sqrt{n}} \vec{J}_{1}\right)(\tau)\right\|_{B_{2,1}^{s}} d \tau .
$$

In the rest of this section, we concentrate on the proof of a priori estimates for a smooth solution $\left(\varrho, \vec{u}, \Theta, j_{0}, \vec{j}_{1}\right)$ to $(116)$, on $[0, T] \times \mathbb{R}^{n}$. We assume in addition that $\varrho(t, x)$ and $\vartheta(t, x)$ are bounded by above and from below for all $(t, x)$ in $[0, T] \times \mathbb{R}^{n}$ :

$$
\begin{aligned}
& 0<\frac{1}{2} \varrho_{*} \leq \varrho(t, x) \leq 2 \varrho^{*}<\infty \\
& \text { and } 0<\frac{1}{2} \vartheta_{*} \leq \vartheta(t, x) \leq 2 \vartheta^{*}<\infty .
\end{aligned}
$$

\footnotetext{
8 Adaptation to nonhomogeneous norms is straightforward, it is only a matter of proving separately an $L^{2}$ inequality for the low-frequency block $\Delta_{-1} \vec{u}$.
} 
Step 1. Estimates for the density Because

$$
\left(\partial_{t}+\vec{u} \cdot \nabla\right) \varrho^{ \pm 1} \pm \varrho^{ \pm 1} \operatorname{div} \vec{u}=0,
$$

we readily have

$$
\left\|\varrho^{ \pm 1}(t)\right\|_{L^{\infty}} \leq e^{\int_{0}^{t}\|\operatorname{div} \vec{u}\|_{L^{\infty}} \mathrm{d} \tau}\left\|\varrho^{ \pm 1}(0)\right\|_{L^{\infty}} .
$$

This implies that (117) if fulfilled if

$$
\varrho_{*} \leq \varrho_{0} \leq \varrho^{*}
$$

and we have some control on $\operatorname{div} \vec{u}$ in $L^{1}\left(0, T ; L^{\infty}\right)$.

Next, differentiating the above equation with respect to the space variable yields

$$
\left(\partial_{t}+\vec{u} \cdot \nabla\right) \nabla \varrho^{ \pm 1}+\nabla \vec{u} \cdot \nabla \varrho^{ \pm 1} \pm \nabla \varrho^{ \pm 1} \operatorname{div} \vec{u} \pm \varrho^{ \pm 1} \nabla \operatorname{div} \vec{u}=0 .
$$

Hence using the fact that $B_{2,1}^{\frac{n}{2}}$ is an algebra, that (see Lemma 7.3)

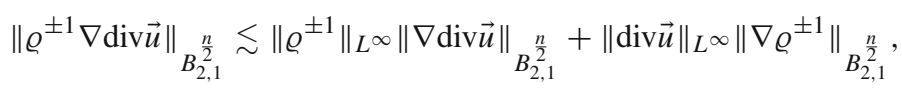

and Proposition 6.1, we obtain

$$
\left\|\nabla \varrho^{ \pm 1}(t)\right\|_{B_{2,1}^{\frac{n}{2}}} \leq e^{C U(t)}\left(\left\|\nabla \varrho^{ \pm 1}(0)\right\|_{B_{2,1}^{\frac{n}{2}}}+C\left\|\varrho^{ \pm 1}\right\|_{L^{\infty}} \int_{0}^{t} e^{-C U(\tau)}\|\nabla \operatorname{div} \vec{u}\|_{B_{2,1}^{\frac{n}{2}}} \mathrm{~d} \tau\right),
$$

with $U(t):=\int_{0}^{t}\|\nabla \vec{u}\|_{B_{2,1}^{\frac{n}{2}}} \mathrm{~d} \tau$.

So assuming from now on that

$$
C U(T) \leq \log 2 \text { and } C \int_{0}^{T}\|\nabla \operatorname{div} \vec{u}\|_{B_{2,1}^{\frac{n}{2}}} d t \leq 2,
$$

we get for all $t \in[0, T]$,

$$
\left\|\nabla \varrho^{ \pm 1}(t)\right\|_{B_{2,1}^{\frac{n}{2}}} \leq A_{0}^{ \pm}:=2\left(\left\|\nabla \varrho^{ \pm 1}(0)\right\|_{B_{2,1}^{\frac{n}{2}}}+2\left\|\varrho^{ \pm 1}\right\|_{L^{\infty}}\right) .
$$

In what follows, we shall denote $A_{0}:=\max \left(A_{0}^{-}, A_{0}^{+}\right)$.

Step 2. Estimates for the radiative functions From Proposition 6.3, we have (omitting $1 / \sqrt{n}$ for notational simplicity):

$$
\begin{aligned}
& \left\|\left(j_{0}, \vec{j}_{1}\right)(t)\right\|_{B_{2,1}^{\frac{n}{2}}} \leq\left\|\left(j_{0}, \vec{j}_{1}\right)(0)\right\|_{B_{2,1}^{\frac{n}{2}}} \\
& \quad+\int_{0}^{t}\left\|\sigma_{a}\left(b(\vartheta)-b(\bar{\vartheta})-j_{0}\right)\right\|_{B_{2,1}^{\frac{n}{2}}} \mathrm{~d} \tau+\int_{0}^{t}\left\|\left(\sigma_{a}+\sigma_{S}\right) \vec{j}_{1}\right\|_{B_{2,1}^{\frac{n}{2}}} \mathrm{~d} \tau .
\end{aligned}
$$

The first term of the r.h.s. may be bounded according to Lemmas 7.3 and 7.4:

$$
\begin{aligned}
& \left\|\sigma_{a}\left(b(\vartheta)-b(\bar{\vartheta})-j_{0}\right)\right\|_{B_{2,1}^{\frac{n}{2}}} \\
& \lesssim\left\|\sigma_{a}\right\|_{L^{\infty}}\left\|b(\vartheta)-b(\bar{\vartheta})-j_{0}\right\|_{B_{2,1}^{\frac{n}{2}}}+\left\|b(\vartheta)-b(\bar{\vartheta})-j_{0}\right\|_{B_{2,1}^{\frac{n}{2}}\left\|\nabla \sigma_{a}\right\|_{B_{2,1}^{\frac{n}{2}-1}}} \\
& \quad\left(\|\Theta\|_{B_{2,1}^{\frac{n}{2}}}+\left\|j_{0}\right\|_{B_{2,1}^{\frac{n}{2}}}\right)\left(1+\|\nabla \varrho\|_{B_{2,1}^{\frac{n}{2}-1}}+\|\nabla \Theta\|_{B_{2,1}^{\frac{n}{2}-1}}\right) .
\end{aligned}
$$


A similar inequality may be proved for the last term of the r.h.s. of (122). Using (121), we thus end up with

$$
\left\|\left(j_{0}, \vec{j}_{1}\right)(t)\right\|_{B_{2,1}^{\frac{n}{2}}} \leq\left\|\left(j_{0}, \vec{j}_{1}\right)(0)\right\|_{B_{2,1}^{\frac{n}{2}}}+C \int_{0}^{t}\left(A_{0}+\|\Theta\|_{B_{2,1}^{\frac{n}{2}}}\right)\left\|\left(\Theta, j_{0}, \vec{j}_{1}\right)\right\|_{B_{2,1}^{\frac{n}{2}}} \mathrm{~d} \tau,
$$

and thus, denoting $J(t):=\left\|\left(j_{0}, \vec{j}_{1}\right)(t)\right\|_{B_{2,1}^{\frac{n}{2}}}$ and using Gronwall Lemma,

$$
J(t) \leq e^{C \int_{0}^{t}\left(A_{0}+\|\Theta\|_{\left.B_{2,1}^{\frac{n}{2}}\right)} \mathrm{d} \tau\right.}\left(J(0)+C \int_{0}^{t}\left(A_{0}+\|\Theta\|_{B_{2,1}^{\frac{n}{2}}}\right)\|\Theta\|_{B_{2,1}^{\frac{n}{2}}} \mathrm{~d} \tau\right) .
$$

Step 3. Estimates for the velocity field Fix some positive real number $\bar{\varrho}$, and set $\bar{\mu}:=\mu(\bar{\varrho}, \bar{\vartheta})$ and $\bar{\lambda}:=\lambda(\bar{\varrho}, \bar{\vartheta})$. Let $\vec{u}_{L}$ be the solution to the following constant coefficients Lamé system:

$$
\partial_{t} \vec{u}_{L}-\overline{\mathcal{A}} \vec{u}_{L}=0,\left.\quad \vec{u}_{L}\right|_{t=0}=\vec{u}_{0} \quad \text { with } \overline{\mathcal{A}}:=\bar{\mu} \Delta+(\bar{\lambda}+\bar{\mu}) \nabla \operatorname{div} .
$$

The fluctuation $\vec{u}_{F}:=\vec{u}-\vec{u}_{L}$ fulfills

$$
\partial_{t} \vec{u}_{F}-\frac{1}{\varrho} \operatorname{div}\left(2 \mu D \vec{u}_{F}+\lambda \operatorname{div} \vec{u}_{F} \text { Id }\right)=\vec{g}_{F},\left.\quad \vec{u}_{F}\right|_{t=0}=0,
$$

with

$$
\vec{g}_{F}:=-\vec{u} \cdot \nabla \vec{u}-\frac{1}{\varrho} \nabla p+\left(\frac{\sigma_{a}+\sigma_{s}}{n \varrho}\right) \vec{j}_{1}+\frac{1}{\varrho} \operatorname{div}\left(2 \mu D \vec{u}_{L}+\lambda \operatorname{div} \vec{u}_{L} \text { Id }\right)-\overline{\mathcal{A}}_{L} .
$$

Applying the second part of Proposition 6.2 yields for some $C=C\left(\varrho_{*}, \varrho^{*}, \lambda, \mu, n\right)$,

$$
X_{\vec{u}_{F}}(t) \leq C \exp \left(C \int_{0}^{t}\left(1+\left\|\mu \nabla \varrho^{-1}, \lambda \nabla \varrho^{-1}, \varrho^{-1} \nabla \mu, \varrho^{-1} \nabla \lambda\right\|_{B_{2,1}^{\frac{n}{2}}}^{2}\right) \mathrm{d} \tau\right) \int_{0}^{t}\left\|\vec{g}_{F}\right\|_{B_{2,1}^{\frac{n}{2}}} \mathrm{~d} \tau,
$$

where we have used the notation

$$
X_{z}(t):=\|z(t)\|_{B_{2,1}^{\frac{n}{2}}}+\int_{0}^{t}\|z\|_{B_{2,1}^{\frac{n}{2}+2}} \mathrm{~d} \tau .
$$

From Lemmas 7.3 and 7.4, we gather that

$$
\begin{aligned}
\left\|\mu \nabla \varrho^{-1}\right\|_{B_{2,1}^{\frac{n}{2}}} & \lesssim\|\mu\|_{L^{\infty}}\left\|\nabla \varrho^{-1}\right\|_{B_{2,1}^{\frac{n}{2}}}+\left\|\varrho^{-1}\right\|_{L^{\infty}\|\nabla \mu\|_{B_{2,1}^{\frac{n}{2}}}} \\
& \lesssim A_{0}\left(1+\|\nabla \varrho, \nabla \Theta\|_{B_{2,1}^{\frac{n}{2}}}\right) \lesssim A_{0}\left(A_{0}+\|\nabla \Theta\|_{B_{2,1}^{\frac{n}{2}}}\right) .
\end{aligned}
$$

A similar bound holds for the other terms of the exponential in (124). Let us now bound the terms of $\vec{g}_{F}$. First, because $B_{2,1}^{\frac{n}{2}}$ is an algebra, we have

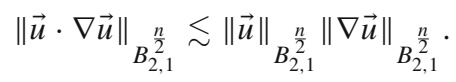

Next, using Lemmas 7.3 and 7.4, Inequality (121) and the expression of $p$ in (9),

$$
\left\|\varrho^{-1} \nabla p\right\|_{B_{2,1}^{\frac{n}{2}}} \lesssim A_{0}\left(1+\|\Theta\|_{B_{2,1}^{\frac{n}{2}+1}}\right) .
$$

Likewise, we get

$$
\left\|\left(\frac{\sigma_{a}+\sigma_{s}}{n \varrho}\right) \vec{j}_{1}\right\|_{B_{2,1}^{\frac{n}{2}}} \lesssim\left(1+A_{0}+\|\Theta\|_{B_{2,1}^{\frac{n}{2}}}\right)\left\|\vec{j}_{1}\right\|_{B_{2,1}^{\frac{n}{2}}}
$$


and

$$
\begin{aligned}
& \left\|\varrho^{-1} \operatorname{div}\left(\mu D \vec{u}_{L}\right)\right\|_{B_{2,1}^{\frac{n}{2}}} \lesssim\left\|\varrho^{-1}\right\|_{L^{\infty}}\left\|\mu D \vec{u}_{L}\right\|_{B_{2,1}^{\frac{n}{2}+1}}+\left\|\mu D \vec{u}_{L}\right\|_{L^{\infty}}\left\|\nabla \varrho^{-1}\right\|_{B_{2,1}^{\frac{n}{2}}}
\end{aligned}
$$

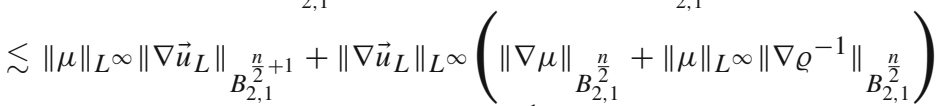

$$
\begin{aligned}
& \lesssim\left\|\nabla \vec{u}_{L}\right\|_{B_{2,1}^{\frac{n}{2}+1}}+\left\|\nabla \vec{u}_{L}\right\|_{B_{2,1}^{\frac{n}{2}}}\left\|\left(\nabla \varrho, \nabla \varrho^{-1}, \nabla \Theta\right)\right\|_{B_{2,1}^{\frac{n}{2}}} .
\end{aligned}
$$

A similar inequality may be proved for $\varrho^{-1} \nabla\left(\lambda \operatorname{div} \vec{u}_{L}\right)$ and we end up, by virtue of (121), with

$$
\| \varrho^{-1} \operatorname{div}\left(2 \mu D \vec{u}_{L}+\lambda \operatorname{div} \vec{u}_{L} \text { Id }\right)\left\|_{B_{2,1}^{\frac{n}{2}}} \lesssim\right\| \nabla \vec{u}_{L}\left\|_{B_{2,1}^{\frac{n}{2}+1}}+\right\| \nabla \vec{u}_{L} \|_{B_{2,1}^{\frac{n}{2}}}\left(A_{0}+\|\nabla \Theta\|_{B_{2,1}^{\frac{n}{2}}}\right) .
$$

Putting all the above inequalities together, we conclude that

$$
\begin{aligned}
& X_{\vec{u}_{F}}(t) \leq C e^{C\left(t\left(1+A_{0}^{2}\right)+\int_{0}^{t}\|\nabla \Theta\|^{2}{ }^{n}{ }_{2,1}^{2}\right.}{ }^{\mathrm{d} \tau)}\left(\sqrt{t} X_{\vec{u}}^{2}(t)+\left(A_{0}+1\right) t J(t)\right. \\
& \left.+\left(X_{\Theta}(t)+A_{0} \sqrt{t}\right)\left(\sqrt{t}\left(1+A_{0}+J(t)\right)+\left\|\nabla \vec{u}_{L}\right\|_{L_{t}^{2}\left(B_{2,1}^{2}\right)^{\frac{n}{2}}}\right)+\left\|\nabla \vec{u}_{L}\right\|_{L_{t}^{1}\left(B_{2,1}^{\frac{n}{2}+1}\right)}\right) .
\end{aligned}
$$

Step 4. Estimates for the temperature Let $\bar{\kappa}:=\frac{\kappa(\bar{\varrho}, \bar{\vartheta})}{C_{v} \bar{\varrho}}$ and $\Theta_{L}$ be the solution to the following constant coefficient heat equation:

$$
\partial_{t} \Theta_{L}-\bar{\kappa} \Delta \Theta_{L}=0,\left.\quad \Theta_{L}\right|_{t=0}=u_{0} .
$$

The fluctuation $\Theta_{F}:=\Theta-\Theta_{L}$ fulfills

$$
\partial_{t} \Theta_{F}-\frac{1}{C_{v} \varrho} \operatorname{div}\left(\kappa \nabla \Theta_{F}\right)=h_{F},\left.\quad \Theta_{F}\right|_{t=0}=0,
$$

with

$$
\begin{aligned}
h_{F}:= & -\vec{u} \cdot \nabla \Theta+\frac{1}{C_{v} \varrho}\left(2 \mu D \vec{u}: D \vec{u}+\lambda(\operatorname{div} \vec{u})^{2}\right)-\frac{1}{C_{v}} \frac{\pi_{1}(\varrho)}{\varrho} \vartheta \operatorname{div} \vec{u} \\
& +\frac{\sigma_{a}}{C_{v} \varrho}\left(j_{0}+b(\bar{\vartheta})-b(\vartheta)\right)+\frac{\left(\sigma_{a}+\sigma_{s}\right)}{C_{v} \varrho} \vec{j}_{1} \cdot \vec{u}+\frac{1}{C_{v} \varrho} \operatorname{div}\left(\kappa \nabla \Theta_{L}\right)-\bar{\kappa} \Delta \Theta_{L} .
\end{aligned}
$$

The maximum principle guarantees that $\vartheta_{*} \leq \bar{\vartheta}+\Theta_{L} \leq \vartheta^{*}$ if

$$
\vartheta_{*} \leq \vartheta_{0} \leq \vartheta^{*} \text {. }
$$

Therefore remembering that $B_{2,1}^{\frac{n}{2}} \hookrightarrow L^{\infty}$, to ensure that (118) is fulfilled, it suffices to establish that $\sup _{t \in[0, T]}\left\|\Theta_{F}(t)\right\|_{B_{2,1}^{\frac{n}{2}}}$ is small enough.

Now applying the first part of Proposition 6.2 yields

$$
X_{\Theta_{F}}(t) \leq C \exp \left(C \int_{0}^{t}\left(1+\left\|\left(\kappa \nabla \varrho^{-1}, \varrho^{-1} \nabla \kappa\right)\right\|_{B_{2,1}^{\frac{n}{2}}}^{2}\right) \mathrm{d} \tau\right) \int_{0}^{t}\left\|h_{F}\right\|_{B_{2,1}^{\frac{n}{2}}} \mathrm{~d} \tau .
$$

As in the previous step, we have

$$
\left\|\left(\kappa \nabla \varrho^{-1}, \varrho^{-1} \nabla \kappa\right)\right\|_{B_{2,1}^{\frac{n}{2}}} \lesssim\left(A_{0}+\|\nabla \Theta\|_{B_{2,1}^{\frac{n}{2}}}\right) A_{0} .
$$


So we now have to bound $h_{F}$ in $B_{2,1}^{\frac{n}{2}}$. Because $B_{2,1}^{\frac{n}{2}}$ is an algebra, we have

$$
\|\vec{u} \cdot \nabla \Theta\|_{B_{2,1}^{\frac{n}{2}}} \lesssim\|\vec{u}\|_{B_{2,1}^{\frac{n}{2}}}\|\nabla \Theta\|_{B_{2,1}^{\frac{n}{2}}} \cdot
$$

Lemmas 7.3 and 7.4 ensure that

$$
\begin{aligned}
\left\|\varrho^{-1} \mu D \vec{u}: D \vec{u}\right\|_{B_{2,1}^{\frac{n}{2}}} & \lesssim\left\|\varrho^{-1} \mu\right\|_{L^{\infty}}\|\nabla \vec{u} \otimes \nabla \vec{u}\|_{B_{2,1}^{\frac{n}{2}}}+\|\nabla \vec{u} \otimes \nabla \vec{u}\|_{B_{2,1}^{\frac{n}{2}-1}}\left\|\nabla\left(\varrho^{-1} \mu\right)\right\|_{B_{2,1}^{\frac{n}{2}}} \\
& \lesssim\|\nabla \vec{u}\|_{B_{2,1}^{\frac{n}{2}}}\left(\|\nabla \vec{u}\|_{B_{2,1}^{\frac{n}{2}}}+\|(\nabla \varrho, \nabla \Theta)\|_{\left.B_{2,1}^{\frac{n}{2}}\|\nabla \vec{u}\|_{B_{2,1}^{\frac{n}{2}-1}}\right) .}\right.
\end{aligned}
$$

Similarly, we have

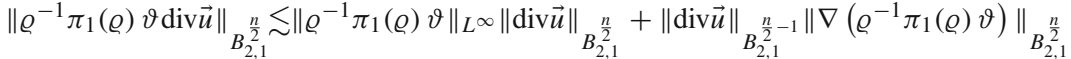

$$
\begin{aligned}
& \lesssim\|\operatorname{div} \vec{u}\|_{B_{2,1}^{\frac{n}{2}}}+\left(A_{0}+\|\nabla \Theta\|_{B_{2,1}^{\frac{n}{2}}}\right)\|\operatorname{div} \vec{u}\|_{B_{2,1}^{\frac{n}{2}-1}}, \\
& \left\|\varrho^{-1} \sigma_{a} j_{0}\right\|_{B_{2,1}^{\frac{n}{2}}} \lesssim\left(1+\|(\nabla \varrho, \nabla \Theta)\|_{\left.B_{2,1}^{\frac{n}{2}-1}\right)} \|_{j_{0} \|_{B_{2,1}^{2}}^{\frac{n}{2}},}\right.
\end{aligned}
$$

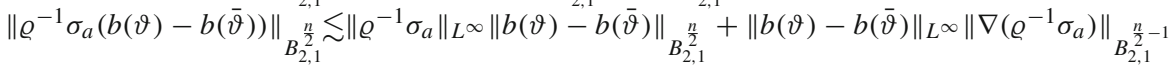

$$
\begin{aligned}
& \lesssim\|\Theta\|_{B_{2,1}^{\frac{n}{2}}}\left(1+\|(\nabla \varrho, \nabla \Theta)\|_{B_{2,1}^{\frac{n}{2}-1}}\right), \\
& \left\|\varrho^{-1}\left(\sigma_{a}+\sigma_{s}\right) \vec{j}_{1} \cdot \vec{u}\right\|_{B_{2,1}^{\frac{n}{2}}} \lesssim\left(1+\|(\nabla \varrho, \nabla \Theta)\|_{\left.B_{2,1}^{\frac{n}{2}-1}\right)}\left\|\vec{j}_{1}\right\|_{B_{2,1}^{\frac{n}{2}}}\|\vec{u}\|_{B_{2,1}^{\frac{n}{2}}}\right.
\end{aligned}
$$

Finally, arguing exactly as for the corresponding term in $\vec{g}_{F}$, we get

$$
\left\|\varrho^{-1} \operatorname{div}\left(\kappa \nabla \Theta_{L}\right)\right\|_{B_{2,1}^{\frac{n}{2}}} \lesssim\left\|\nabla \Theta_{L}\right\|_{B_{2,1}^{\frac{n}{2}+1}}+\left\|\nabla \Theta_{L}\right\|_{B_{2,1}^{\frac{n}{2}}}\left(A_{0}+\| \nabla \Theta_{B_{2,1}^{\frac{n}{2}}}\right) .
$$

Putting together all the previous inequalities, we conclude that

$$
\begin{aligned}
& X_{\Theta_{F}}(t) \leq C e^{C\left(\left(A_{0}^{2}+1\right) t+\int_{0}^{t} \| \nabla \Theta^{2}{ }_{B_{2,1}^{n}}^{2}\right.}{ }^{\mathrm{d} \tau)}\left(\left\|\nabla \Theta_{L}\right\|_{L_{t}^{2}\left(B_{2,1}^{\frac{n}{2}}\right)}^{2}+\left\|\nabla \Theta_{L}\right\|_{L_{t}^{1}\left(B_{2,1}^{\frac{n}{2}+1}\right)} A_{0}\right. \\
& +X_{\vec{u}_{F}}^{2}+X_{\Theta_{F}}^{2}+A_{0} \sqrt{t} X_{\vec{u}}^{2}+X_{\vec{u}}\left(X_{\vec{u}_{F}}+\left\|\nabla \vec{u}_{L}\right\|_{L_{t}^{2}\left(B_{2,1}^{\frac{n}{2}}\right)}\right)\left(X_{\Theta_{F}}+\left\|\nabla \Theta_{L}\right\|_{L^{2}\left(B_{2,1}^{\frac{n}{2}}\right)}\right) \\
& +\left(\sqrt{t}+\sqrt{t} X_{\Theta}+A_{0} t\right) X_{\vec{u}}+t\left(1+A_{0}+X_{\Theta}\right)\left(X_{\Theta}+J\left(1+X_{\vec{u}}\right)\right) \\
& \left.+\sqrt{t} A_{0}\left\|\nabla \Theta_{L}\right\|_{L_{t}^{2}\left(B_{2,1}^{\frac{n}{2}}\right)}\right) .
\end{aligned}
$$

Step 5. Closure of the estimates Let $T^{*} \leq T$ be the largest time for which

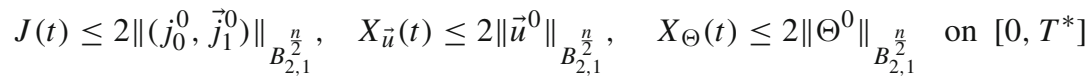

and, in addition,

$$
\begin{array}{ll}
X_{\vec{u}_{F}}\left(T^{*}\right) \leq \eta_{\vec{u}}, & X_{\vec{u}_{L}}\left(T^{*}\right) \leq \eta_{\Theta}, \\
\left\|\nabla \vec{u}_{L}\right\|_{L_{T^{*}}^{1}\left(B_{2,1}^{\frac{n}{2}+1}\right)} \leq \varepsilon_{1}, & \left\|\nabla \vec{u}_{L}\right\|_{L_{T^{*}}^{2}\left(B_{2,1}^{\frac{n}{2}}\right)} \leq \varepsilon_{2}, \\
\left\|\nabla \Theta_{L}\right\|_{L_{T^{*}}^{1}\left(B_{2,1}^{\frac{n}{2}+1}\right)} \leq \widetilde{\varepsilon}_{1}, & \left\|\nabla \Theta_{L}\right\|_{L_{T^{*}}^{2}\left(B_{2,1}^{\frac{n}{2}}\right)} \leq \widetilde{\varepsilon}_{2},
\end{array}
$$

for suitably small $\eta_{\vec{u}}, \eta_{\Theta}, \varepsilon_{1}, \varepsilon_{2}, \widetilde{\varepsilon}_{1}$ and $\widetilde{\varepsilon}_{2}$ (that will be fixed hereafter). Note that the time continuity properties of the solution and the fact that $\nabla \vec{u}_{L}, \nabla \Theta_{L} \in L_{l o c}^{1}\left(\mathbb{R}^{+} ; B_{2,1}^{\frac{n}{2}+1}\right)$ and that $\nabla \vec{u}_{L}, \nabla \Theta_{L} \in L_{l o c}^{2}\left(\mathbb{R}^{+} ; B_{2,1}^{\frac{n}{2}}\right)$ ensures that $T^{*}>0$, as well as (117) and (118) if the data fulfill (120) and (126). 
Therefore from Inequality (123) we deduce that for all $t \in\left[0, T^{*}\right]$,

$$
J(t) \leq e^{C\left(A_{0} t+2 \sqrt{t}\left\|\Theta^{0}\right\|{ }_{B_{2,1}^{2}}^{\frac{n}{2}}\right)}\left(\left\|\left(j_{0}^{0}, \vec{j}_{1}^{0}\right)\right\|_{B_{2,1}^{\frac{n}{2}}}+2 C t\left(A_{0}+2\left\|\Theta^{0}\right\|_{B_{2,1}^{\frac{n}{2}}}\right)\left\|\Theta^{0}\right\|_{B_{2,1}^{\frac{n}{2}}}\right) .
$$

Hence $J(t) \leq 2\left\|\left(j_{0}^{0}, \vec{j}_{1}^{0}\right)\right\|_{B_{2,1}^{\frac{n}{2}}}$ on $\left[0, T^{*}\right]$ whenever (say)

$$
\begin{aligned}
C\left(A_{0} T^{*}+2 \sqrt{T^{*}}\left\|\Theta^{0}\right\|_{B_{2,1}^{\frac{n}{2}}}\right) & \leq \log \frac{3}{2} \text { and } 6 C T^{*}\left(A_{0}+2\left\|\Theta^{0}\right\|_{B_{2,1} \frac{n}{2}}\right)\left\|\Theta^{0}\right\|_{B_{2,1}^{\frac{n}{2}}} \\
& \leq\left\|\left(j_{0}^{0}, \vec{j}_{1}^{0}\right)\right\|_{B_{2,1}^{\frac{n}{2}}}
\end{aligned}
$$

Next, if we assume in addition that

$$
\left(\left(A_{0}^{2}+1\right) T^{*}+\int_{0}^{T^{*}}\|\nabla \Theta\|_{B_{2,1}^{\frac{n}{2}}}^{2} d t\right) \text { is small enough }
$$

then Inequality (125) implies that on $\left[0, T^{*}\right]$, we have

$$
X_{\vec{u}_{F}}(t) \leq 4 C\left(\sqrt{t}\left\|\vec{u}^{0}\right\|_{B_{2,1}^{\frac{n}{2}}}^{2}+\left(\left\|\Theta_{0}\right\|_{B_{2,1}^{\frac{n}{2}}}+\sqrt{t} A_{0}\right)\left(\sqrt{t}\left(\left\|\left(j_{0}^{0}, \vec{j}_{1}^{0}\right)\right\|_{B_{2,1}^{\frac{n}{2}}}+A_{0}+1\right)+\varepsilon_{2}\right)+\varepsilon_{1}\right) .
$$

And finally, from (128), we infer that

$$
\begin{aligned}
X_{\Theta_{F}}(t) \leq & 4 C\left(X_{\vec{u}_{F}}^{2}+A_{0} \sqrt{t}\left\|\vec{u}^{0}\right\|_{B_{2,1}^{\frac{n}{2}}}^{2}+\left\|\vec{u}^{0}\right\|_{B_{2,1}^{\frac{n}{2}}}\left(X_{\vec{u}_{F}}+\varepsilon_{2}\right)\left(X_{\Theta_{F}}+\widetilde{\varepsilon}_{2}\right)\right. \\
& +\sqrt{t}\left\|\vec{u}^{0}\right\|_{B_{2,1}^{\frac{n}{2}}}\left(1+\left\|\Theta_{0}\right\|_{B_{2,1}^{\frac{n}{2}}}+\sqrt{t} A_{0}\right)+t\left(1+A_{0}+\left\|\Theta^{0}\right\|_{B_{2,1}^{\frac{n}{2}}}\right)\left(\left\|\Theta^{0}\right\|_{B_{2,1}^{\frac{n}{2}}}\right. \\
& \left.+\left\|\left(j_{0}^{0}, \vec{j}_{1}^{0}\right)\right\|_{B_{2,1}^{\frac{n}{2}}}\left(1+\left\|\vec{u}^{0}\right\|_{B_{2,1}^{\frac{n}{2}}}\right)\right) \\
& \left.+A_{0} \widetilde{\varepsilon}_{1}+\widetilde{\varepsilon}_{2}^{2}+X_{\Theta_{F}}^{2}+\sqrt{t} A_{0} \widetilde{\varepsilon}_{2}\right) .
\end{aligned}
$$

To conclude, it suffices to notice that for given $\eta_{\vec{u}}>0$, then after taking $\varepsilon_{1}$ and $\varepsilon_{2}$ small enough, Inequality (133) will guarantee that $X_{\vec{u}} \leq \eta_{\vec{u}}$ on a small time interval the length of which may be bounded by below in terms of $\eta_{\vec{u}}$ and of the norms of the data. Let us underscore that imposing the values of $\varepsilon_{1}$ and $\varepsilon_{2}$ may be converted into a (not so explicit) smallness condition $T^{*}$, through the whole function $\vec{u}^{0}$.

Similarly, taking $\widetilde{\varepsilon}_{1}$ and $\widetilde{\varepsilon}_{2}$ small enough, (134) will imply that $X_{\Theta_{F}} \leq \eta_{\Theta}$ on some time interval depending only on the data.

To complete the proof of a priori estimates, we still have to check that (129) and (132) are indeed fulfilled if $t, \eta_{\vec{u}}$ and $\eta_{\Theta}$ have been chosen sufficiently small. Given that $X_{\vec{u}} \leq$ $X_{\vec{u}_{L}}+X_{\vec{u}_{F}}, X_{\Theta} \leq X_{\Theta_{L}}+X_{\Theta_{F}}, X_{\vec{u}_{L}}(t) \leq(1+t)\left\|\vec{u}^{0}\right\|_{B_{2,1}^{\frac{n}{2}}}$ and $X_{\Theta_{L}}(t) \leq(1+t)\left\|\Theta^{0}\right\|_{B_{2,1} \frac{n}{2}}$, Inequality (129) is fulfilled if $t$ is small enough and

$$
\eta_{\vec{u}} \leq \frac{1}{2}\left\|\vec{u}^{0}\right\|_{B_{2,1}^{\frac{n}{2}}} \text { and } \eta_{\Theta} \leq \frac{1}{2}\left\|\Theta^{0}\right\|_{B_{2,1}^{\frac{n}{2}}} .
$$

Finally, we have

$$
\int_{0}^{t}\|\nabla \Theta\|_{B_{2,1}^{2}}^{2} \mathrm{~d} \tau \leq 2 \widetilde{\varepsilon}_{2}^{2}+2 \eta_{\Theta}^{2}
$$


hence (132) is fulfilled if $t, \widetilde{\varepsilon}_{2}$ and $\eta_{\Theta}$ have been chosen small enough. Therefore there exists a positive time $T^{*}$ that may be computed in terms of the norms of the data, and of the free solutions $\vec{u}_{L}$ and $\Theta_{L}$ so that (129) is fulfilled on $\left[0, T^{*}\right]$. This completes the proof of a priori estimates in the case of large data and general coefficients.

\section{Besov spaces and Littlewood-Paley decomposition}

Here we shortly recall the definition of Besov spaces, paraproduct, and a few nonlinear estimates that have been used extensively in the paper. The reader will find more material in the textbooks [1] and [27].

Let $\chi: \mathbb{R}^{n} \rightarrow[0,1]$ be a smooth nonincreasing radial function supported in $B(0,1)$ with, additionally, $\chi \equiv 1$ on $B(0,1 / 2)$. Let $\varphi(\xi):=\chi(\xi / 2)-\chi(\xi)$. We define the nonhomogeneous Littlewood-Paley decomposition by setting

$$
\Delta_{-1} u:=\chi(D) u=\mathcal{F}^{-1}(\chi \mathcal{F} u) \text { and } \Delta_{k} u:=\varphi\left(2^{-k} D\right) u=\mathcal{F}^{-1}\left(\varphi\left(2^{-k} \cdot\right) \mathcal{F} u\right) \text { if } k \geq 0 .
$$

Note that by construction,

$$
\chi+\sum_{k \geq 0} \varphi\left(2^{-k} \cdot\right) \equiv 1 \quad \text { in } \mathbb{R}^{n}
$$

and thus

$$
\sum_{k \geq-1} \Delta_{k}=\text { Id holds in } \mathcal{S}^{\prime}\left(\mathbb{R}^{n}\right)
$$

Likewise, we define the homogeneous Littlewood-Paley decomposition by

$$
\dot{\Delta}_{k} u:=\varphi\left(2^{-k} D\right) u=\mathcal{F}^{-1}\left(\varphi\left(2^{-k} \cdot\right) \mathcal{F} u\right) \text { for all } k \in \mathbb{Z} .
$$

Because

$$
\sum_{k \in \mathbb{Z}} \varphi\left(2^{-k} \xi\right)=1 \text { if and only if } \xi \neq 0,
$$

in contrast with its nonhomogeneous counterpart, we only have

$$
\sum_{k \in \mathbb{Z}} \dot{\Delta}_{k}=\text { Id in } \mathcal{S}^{\prime}\left(\mathbb{R}^{n}\right) \text { modulo polynomials. }
$$

Definition 7.1 For $s \in \mathbb{R}$ we set

$$
\|u\|_{B_{2,1}^{s}}:=\sum_{k \geq-1} 2^{s k}\left\|\Delta_{k} u\right\|_{L^{2}} \text { and }\|u\|_{\dot{B}_{2,1}^{s}}:=\sum_{k \in \mathbb{Z}} 2^{s k}\left\|\dot{\Delta}_{k} u\right\|_{L^{2}} .
$$

The nonhomogeneous Besov space $B_{2,1}^{s}=B_{2,1}^{s}\left(\mathbb{R}^{n}\right)$ is the set of tempered distributions $u$ so that $\|u\|_{B_{2,1}^{s}}<\infty$, while the homogeneous Besov space $\dot{B}_{2,1}^{s}=\dot{B}_{2,1}^{s}\left(\mathbb{R}^{n}\right)$ is the set of tempered distributions $u$ so that $\|u\|_{\dot{B}_{2,1}^{s}}<\infty$ and, in addition ${ }^{9}$

$$
\lim _{\lambda \rightarrow+\infty} \theta(\lambda D) u=0 \text { in } L^{\infty}
$$

for some test function $\theta$ with $\theta(0) \neq 0$.

9 That latter condition ensures that $u=\sum_{j \in \mathbb{Z}} \dot{\Delta}_{j} u$ in $\mathcal{S}^{\prime}\left(\mathbb{R}^{n}\right)$. 
As in $[5,7,12]$, working with different Besov norms for low and high frequencies is the key to the proof of global results. This motivates our introducing the notation

$$
\|u\|_{\dot{B}_{2,1}^{s}}^{\ell}:=\sum_{k \leq k_{0}} 2^{k s}\left\|\dot{\Delta}_{k} u\right\|_{L^{2}} \quad \text { and } \quad\|u\|_{\dot{B}_{2,1}^{s}}^{h}:=\sum_{k>k_{0}} 2^{k s}\left\|\dot{\Delta}_{k} u\right\|_{L^{2}},
$$

where $k_{0} \in \mathbb{Z}$ is a fixed integer depending only on the coefficients of System (25).

Likewise, we shall use the notation

$$
u^{\ell}:=\sum_{k \leq k_{0}} \dot{\Delta}_{k} u \text { and } u^{h}:=\sum_{k>k_{0}} \dot{\Delta}_{k} u .
$$

The paraproduct between two tempered distributions $u$ and $v$ is defined by

$$
T_{u} v:=\sum_{k} \dot{S}_{k-1} u \dot{\Delta}_{k} v \text { with } S_{k-1}:=\chi\left(2^{k-1} D\right) .
$$

For sufficiently smooth functions (or distributions) the following Bony decomposition holds true:

$$
u v=T_{u} v+R(u, v)+T_{v} u,
$$

where the remainder operator is defined by

$$
R(u, v):=\sum_{k} \dot{\Delta}_{k} u\left(\dot{\Delta}_{k-1} v+\dot{\Delta}_{k} v+\dot{\Delta}_{k+1} v\right) .
$$

The paraproduct and remainder operators are continuous in a number of classical functional spaces. In the present paper, we need the following result:

Lemma 7.2 Let $s$ and $t$ be two real numbers.

- If $t \leq n / 2$ then $T: \dot{B}_{2,1}^{t} \times \dot{B}_{2,1}^{s} \rightarrow \dot{B}_{2,1}^{s+t-\frac{n}{2}}$.

- If $0<s+t \leq n$ then $R: \dot{B}_{2,1}^{t} \times \dot{B}_{2,1}^{s} \rightarrow \dot{B}_{2,1}^{s+t-\frac{n}{2}}$.

The following product laws in Besov spaces (or their homogeneous analogue) have been used a number of times in the paper.

Lemma 7.3 If $s, t>0$ then

$$
\|a u\|_{B_{2,1}^{s}} \lesssim\|a\|_{L^{\infty}}\|u\|_{B_{2,1}^{s}}+\|u\|_{B_{\infty, \infty}^{-t}}\|\nabla a\|_{B_{2,1}^{s-1+t}}
$$

where $\|u\|_{B_{\infty, \infty}^{-t}}:=\sup _{k \geq-1} 2^{-k}\left\|\Delta_{k} u\right\|_{L^{\infty}}$.

Moreover

$$
\|a u\|_{B_{2,1}^{s}} \lesssim\|a\|_{L^{\infty}}\|u\|_{B_{2,1}^{s}}+\|u\|_{L^{\infty}}\|\nabla a\|_{B_{2,1}^{s-1}}
$$

and, for $j=1, \ldots, n$,

$$
\left\|a \partial_{j} v\right\|_{B_{2,1}^{s}} \lesssim\|a\|_{L^{\infty}}\left\|\partial_{j} v\right\|_{B_{2,1}^{s}}+\|v\|_{L^{\infty}}\|\nabla a\|_{B_{2,1}^{s}} .
$$

Proof Use Bony's decomposition

$$
a u=T_{a} u+R(a, u)+T_{u} a,
$$

and the fact that, as in the paraproduct $T_{u} a$ the low frequencies of $a$ are not involved, one may replace $\|a\|_{B_{2,1}^{s+t}}$ with $\|\nabla a\|_{B_{2,1}^{s-1+t}}$ (see Remark 2.83 in [1]). The last inequality stems from the first one with $t=1$, once noticed that $\left\|\partial_{j} v\right\|_{B_{\infty, \infty}^{-1}} \lesssim\|v\|_{L^{\infty}}$ (a consequence of Bernstein inequality). 
Lemma 7.4 Let $s>0$. Let $F: \mathbb{R} \rightarrow \mathbb{R}$ be a smooth function vanishing at 0 . Then we have

$$
\|F(a)\|_{B_{2,1}^{s}} \leq C\left(\|a\|_{L^{\infty}}\right)\|a\|_{B_{2,1}^{s}} \text { and }\|\nabla(F(a))\|_{B_{2,1}^{s-1}} \leq C\left(\|a\|_{L^{\infty}}\right)\|\nabla a\|_{B_{2,1}^{s-1}} .
$$

More generally, if $G: \mathbb{R}^{2} \rightarrow \mathbb{R}$ is a smooth function vanishing at 0 then

$$
\|G(a, \theta)\|_{B_{2,1}^{s}} \leq C\left(\|a\|_{L^{\infty}},\|\theta\|_{L^{\infty}}\right)\|(a, \theta)\|_{B_{2,1}^{s}}
$$

and

$$
\|\nabla(G(a, \theta))\|_{B_{2,1}^{s-1}} \leq C\left(\|a\|_{L^{\infty}},\|\theta\|_{L^{\infty}}\right)\|(\nabla a, \nabla \theta)\|_{B_{2,1}^{s-1}} .
$$

The statements with no gradient are completely standard (see, e.g., [27], Chap. 5). The first refined inequality with a gradient may be found in [10], Prop. 4. Its extension to two variables is not a big deal.

\section{References}

1. Bahouri, H., Chemin, J.-Y., Danchin, R.: Fourier Analysis and Nonlinear Partial Differential Equations. Grundlehren der mathematischen Wissenschaften, vol. 343. Springer, Berlin (2011)

2. Blanc, X., Després, B.: Numerical methods for inertial confinement fusion. Lecture Notes of CEMRACS10 Summer School. http://smai.emath.fr/cemracs/cemracs10/fr_courses.html

3. Buet, C., Després, B.: Asymptotic analysis of fluid models for the coupling of radiation and hydrodynamics. J. Quant. Spectrosc. Radiat. Transf. 85, 385-480 (2004)

4. Chandrasekhar, S.: Radiative Transfer. Dover Publications, New York (1960)

5. Danchin, R.: Global existence in critical spaces for compressible Navier-Stokes equations. Invent. Math. 141, 579-614 (2000)

6. Danchin, R.: Local theory in critical spaces for compressible viscous and heat-conductive gases. Commun. Partial Differ. Equ. 26, 1183-1233 (2001)

7. Danchin, R.: Global existence in critical spaces for flows of compressible viscous and heat-conductive gases. Arch. Ration. Mech. Anal. 160, 1-39 (2001)

8. Danchin, R.: On the uniqueness in critical spaces for compressible Navier-Stokes equations. NoDEA Nonlinear Differ. Equ. Appl. 12(1), 111-128 (2005)

9. Danchin, R.: Well-posedness in critical spaces for barotropic viscous fluids with truly nonconstant density. Commun. Partial Differ. Equ. 32, 1373-1397 (2007)

10. Danchin, R.: On the well-posedness of the incompressible density-dependent Euler equations in the $L^{p}$ framework. J. Differ. Equ. 248, 2130-2170 (2010)

11. Danchin, R.: A Lagrangian approach for the compressible Navier-Stokes equations. Ann. Inst. Fourier 64(2), 753-791 (2014)

12. Danchin, R., Ducomet, B.: On a simplified model for radiating flows. J. Evol. Equ. 14, 155-195 (2013)

13. Danchin, R., Ducomet, B.: The low match number limit for a barotropic model of radiative flow. SIAM J. Math. Anal. 48(2),1025-1053 (2016). doi:10.1137/15M1009081

14. Danchin, R., Ducomet, B.: Diffusive limits for a barotropic model of radiative flow. Conflu. Math. (to appear)

15. Ducomet, B., Feireisl, E., Nečasová, Š.: On a model in radiation hydrodynamics. Ann. Inst. Henri Poincaré Nonlinear Anal. 28, 797-812 (2011)

16. Dubroca, B., Feugeas, J.-L.: Etude théorique et numérique d'une hiérarchie de modèles aux moments pour le transfert radiatif. C. R. Acad. Sci. Paris 329, 915-920 (1999)

17. Frank, M.: Approximate models for radiative transfer. Bull. Inst. Math. Acad. Sin. 2, 409-432 (2007)

18. Gantmacher, F.R.: Théorie des Matrices. Dunod, Paris (1966)

19. Golse, F., Perthame, B.: Generalized solutions of the radiative transfer equations in a singular case. Commun. Math. Phys. 106(2), 211-239 (1986)

20. Levermore, C.D.: Entropy-based moment closures for kinetic equations. Transp. Theory Stat. Phys. 26, 591-606 (1997)

21. Liénard, A., Chipart, H.: Sur le signe de la partie réelle des racines d'une équation algébrique. J. Math. Pures Appl. 10, 291-346 (1914)

22. Lin, C.: Mathematical analysis of radiative transfer models. PhD Thesis (2007) 
23. Lin, C., Coulombel, J.-F., Goudon, T.: Shock profiles for non-equilibrium radiative gases. Phys. D 218, 83-94 (2006)

24. Lowrie, R.B., Morel, J.E., Hittinger, J.A.: The coupling of radiation and hydrodynamics. Astrophys. J. 521, 432-450 (1999)

25. Mihalas, B., Weibel-Mihalas, B.: Foundations of Radiation Hydrodynamics. Dover Publications, Dover (1984)

26. Pomraning, G.C.: Radiation Hydrodynamics. Dover Publications, New York (2005)

27. Runst, T., Sickel, W.: Sobolev Spaces of Fractional Order, Nemytskij Operators, and Nonlinear Partial Differential Equations, Nonlinear Analysis and Applications, 3. Walter de Gruyter \& Co., Berlin (1996) 